\title{
Ultrafast X-ray Science Facility at the Advanced Light Source
}

\section{Principal Investigators:}

Robert W. Schoenlein

Staff Scientist, Materials Sciences Division

Lawrence Berkeley National Laboratory

1 Cyclotron Rd. MS: 2-300

Berkeley, CA 94720

(510) 486-6557, FAX: (510) 486-5530

email: rwschoenlein@lbl.gov

\author{
Roger W. Falcone \\ Professor of Physics \\ Physics Department \\ University of California, Berkeley \\ Berkeley, CA 94720 \\ (510) 642-3316 \\ email: rwf@physics.berkeley.edu
}

\section{Co-investigators}

\section{Scientific Program - (core research team)}

R. Abela

A.P. Alivisatos

A. Belkacem

N. Berrah

J. Bozek

C. Bressler

A. Cavalleri

M. Chergui

T.E. Glover

P.A. Heimann

J. Hepburn

J. Larsson

R.W. Lee

J. McCusker

H.A. Padmore

P. Pattison

S.T. Pratt

C.V. Shank

J. Wark
Swiss Light Source

Chemistry Department

Materials Sciences Division

Chemical Sciences Division

Physics Department

Advanced Light Source

Institute of Condensed Matter Physics

Materials Sciences Division

Institute of Condensed Matter Physics

Advanced Light Source

Advanced Light Source

Department of Chemistry

Physics Department

Physics Division

Chemistry Department

Advanced Light Source

Institute of Crystallography

Chemistry Division

Chemistry Department

Materials Sciences Division

Physics Department
Paul Scherrer Institute, Switzerland University of California, Berkeley

Lawrence Berkeley National Laboratory

Lawrence Berkeley National Laboratory

University of Western Michigan

Lawrence Berkeley National Laboratory

University of Lausanne, Switzerland

Lawrence Berkeley National Laboratory

University of Lausanne, Switzerland

Lawrence Berkeley National Laboratory

Lawrence Berkeley National Laboratory

University of Waterloo, Canada

Lund University, Sweden

Lawrence Livermore National Laboratory

Michigan State University

Lawrence Berkeley National Laboratory

University of Lausanne, Switzerland

Argonne National Laboratory

University of California, Berkeley

Lawrence Berkeley National Laboratory

Oxford University, England

\section{Technical Development}

Z. Chang

D.S. Robin

R.D. Schlueter

A.A. Zholents

M.S. Zolotorev
Center for Ultrafast Optical Science

Advanced Light Source

Engineering Division

Accelerator and Fusion Research Division

Accelerator and Fusion Research Division

\author{
University of Michigan \\ Lawrence Berkeley National Laboratory \\ Lawrence Berkeley National Laboratory \\ Lawrence Berkeley National Laboratory \\ Lawrence Berkeley National Laboratory
}




\section{Table of Contents}

Executive Summary

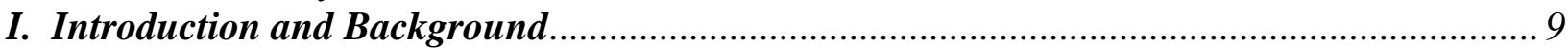

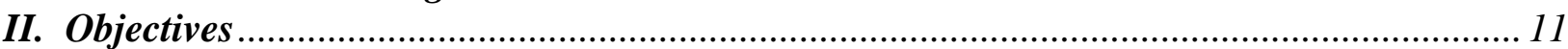

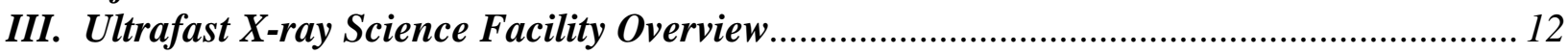

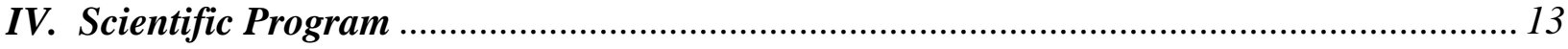

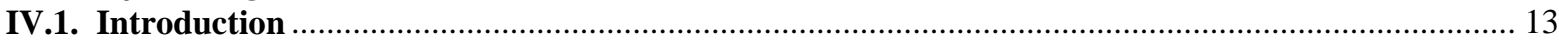

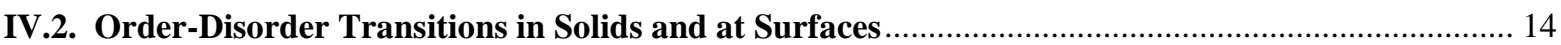

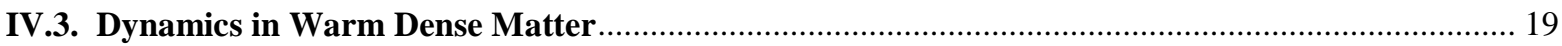

IV.4. Solid-Solid Structural Changes and Phase Transitions .................................................................. 24

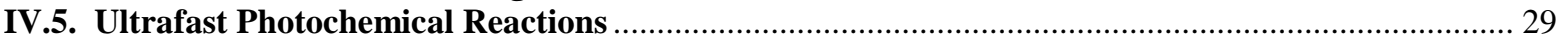

IV.6. Atomic and Molecular Physics with Femtosecond Optical and X-ray Pulses ................................... 35

V. Relation to U.S. Department of Energy Programs.......................................................... 42

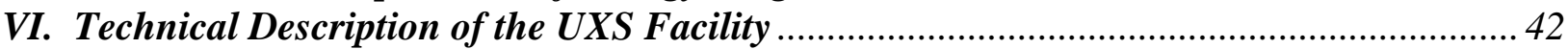

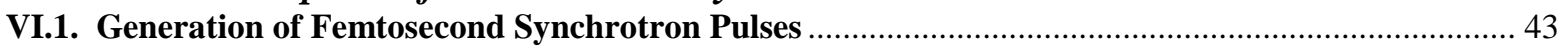

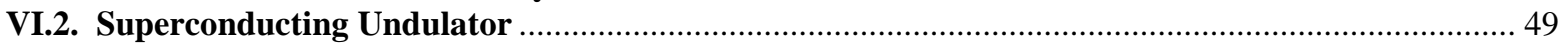

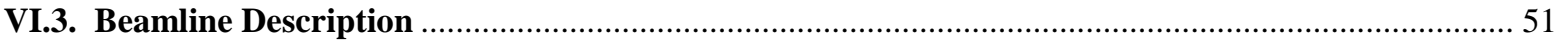

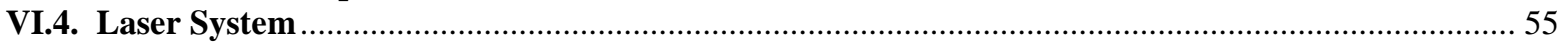

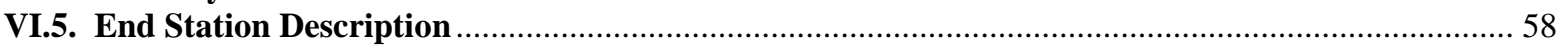

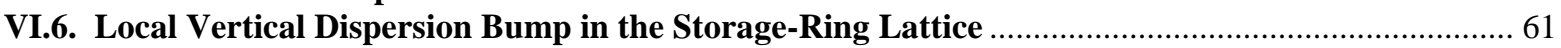

VII. Background and Comparison with Existing Femtosecond X-ray Sources..................... 62

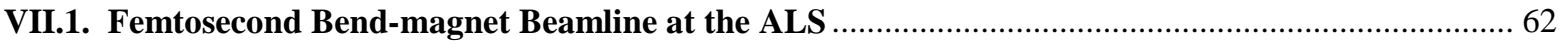

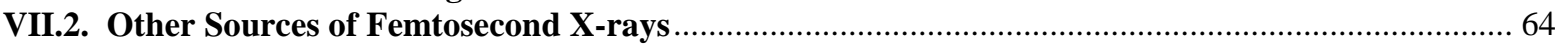

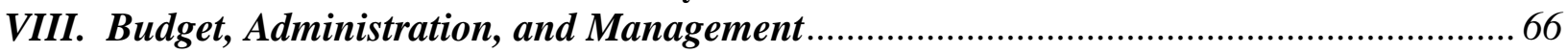

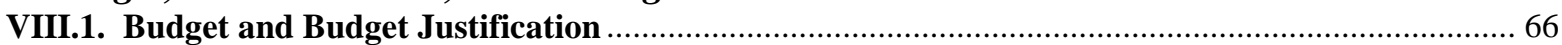

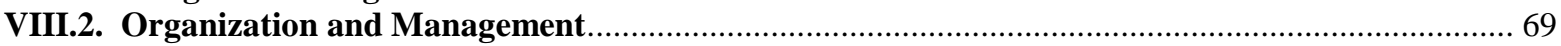

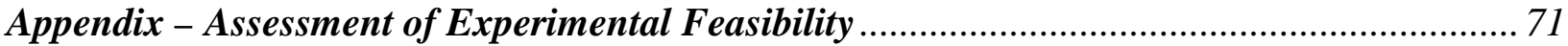

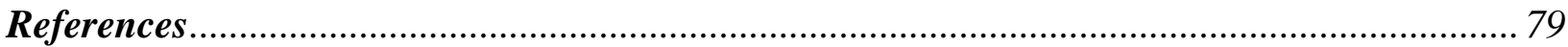

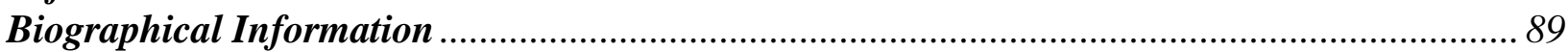




\section{Ultrafast X-ray Science Facility at the Advanced Light Source}

\section{Executive Summary}

We propose to develop a true user facility for ultrafast x-ray science at the Advanced Light Source. This facility will be unique in the world, and will fill a critical need for the growing ultrafast $\mathrm{x}$-ray research community. The development of this facility builds upon the expertise from long-standing research efforts in ultrafast $\mathrm{x}$-ray spectroscopy and the development of femtosecond x-ray sources and techniques at both the Lawrence Berkeley National Laboratory and at U.C. Berkeley. In particular, the technical feasibility of a femtosecond x-ray beamline at the ALS has already been demonstrated, and existing ultrafast laser technology will enable such a beamline to operate near the practical limit for femtosecond $\mathrm{x}$-ray flux and brightness from a $3^{\text {rd }}$ generation synchrotron.

\section{Facility Description}

The Ultrafast X-ray Science (UXS) facility will generate $200 \mathrm{fs} x$-ray pulses in the $0.2-10 \mathrm{keV}$ range with an average flux of $\sim 10^{8} \mathrm{ph} / \mathrm{s} / 0.1 \% \mathrm{BW}$ and an average brightness of $\sim 10^{11} \mathrm{ph} / \mathrm{s} / \mathrm{mm}^{2} / \mathrm{mrad}^{2} / 0.1 \% \mathrm{BW}$. The facility will consist of four main parts: (1) a superconducting undulator source which will provide the highest possible photon flux and brightness in the $0.2-10 \mathrm{keV}$ range, (2) an x-ray beamline consisting of imaging optics to provide a tunable femtosecond $\mathrm{x}$-ray beam in a micro-focus spot, with additional capability for dispersive measurements in the soft x-ray range (3) a high-repetition rate, high-average-power femtosecond laser system and associated beam delivery optics which will serve the dual purpose of providing laser pulses for modulating the synchrotron beam (for generation of femtosecond x-rays) as well as tunable "pump" pulses for sample excitation, and (4) endstation components including an ultrafast streak-camera for measurements on picosecond time scales, a UHV vacuum chamber with a photoelectron spectrometer, and gated detectors ( $2 \mathrm{nsec}$ gate width) for isolating individual synchrotron pulses. The UXS facility will make use of an existing wiggler (which will simultaneously serve the existing protein crystallography beamline) for generating femtosecond $\mathrm{x}$-rays via laser-modulation (slicing) of the stored electron beam.

\section{Scientific Program}

Our scientific understanding of the static or time-averaged structure of matter on the atomic scale has been dramatically advanced by direct structural measurements using x-ray techniques and modern synchrotron sources. Of course the structure of matter is not static, and to understand the behavior of matter at the most fundamental level requires structural measurements on the time scale on which atoms move. The evolution of matter structure is dictated by the making and breaking of chemical bonds and the rearrangement of atoms, and this occurs on the fundamental time scale of a vibrational period, $\sim 100 \mathrm{fs}$. Atomic motion on this time scale ultimately determines the course of phase transitions in solids, the kinetic pathways of chemical reactions, and even the efficiency and function of biological processes. A thorough understanding of such dynamic behavior is a first step to being able to control structural 
evolution, and will have important scientific applications in solid-state physics, chemistry, materials science, AMO physics, and biology.

Our ability to measure and understand fundamental structural dynamics is limited by lack of appropriate experimental tools. Femtosecond lasers provide the requisite time resolution, and have been applied to study ultrafast processes in numerous fields of research. The award of the 1999 Nobel Prize in Chemistry for the application of femtosecond lasers to the study of chemical dynamics underscores the fundamental significance of this time regime. In condensed matter systems however, visible photons from lasers are not effective as structural probes since they interact with electronic states extending over multiple atoms. On the other hand, $\mathrm{x}$-ray photons can provide the requisite structural information via interaction with localized core levels. This capability is driving the emergence of ultrafast $\mathrm{x}$-ray science as a new field of research in which $\mathrm{x}$-ray techniques are used in combination with femtosecond lasers and applied in a time-resolved manner to probe structural dynamics. The scientific potential of this research area is undeveloped due to the inadequacy of present short-pulse x-ray sources.

The scientific development of the UXS facility is driven by a cross-disciplinary core research group with backgrounds in solid-state physics, chemistry, materials science, and AMO physics; and with specific expertise in ultrafast $\mathrm{x}$-ray research and synchrotron research. This scientific expertise is complemented with technical expertise in laser-electron beam interactions, beam physics, femtosecond lasers, and synchrotron instrumentation. The scientific program for the UXS facility is organized around the following five general areas of time-resolved x-ray research.

\section{Order-Disorder Transitions in Solids and at Surfaces}

The dynamics of phase transformations in solids are of fundamental scientific interest since they determine the genesis and evolution of new structural phases. The conventional description of first-order solid/liquid transitions is based on a local thermal equilibrium model which describes the transfer of energy to the lattice in the form of heat. However, femtosecond optical excitation can create highly non-equilibrium conditions by depositing energy in solids faster than the relevant relaxation and dissipation times. Under such conditions, the fundamental structural dynamics are revealed. In principle, non-equilibrium excitation can give rise to the development of unique structural phases. Femtosecond x-ray diffraction provides direct information about the changes in long-range order in crystalline systems. Experiments to date have revealed the physics of energy transfer which begins with the optical excitation of carriers and eventually leads to the generation of coherent acoustic phonons and the development of strain. At sufficiently high excitation densities, the first suggestions of direct disordering on a sub-picosecond time scale have been observed. More sensitive measurements will elucidate the unique physics in this regime. Femtosecond EXAFS and XANES measurements will be used to probe bond-distances and coordination during the transition from solid to liquid, and may identify metastable intermediate phases. These techniques can provide surface sensitivity and will be applicable to order-disorder transitions in polycrystalline and nanostructure systems. 


\section{Dynamics in Warm Dense Matter}

A significant gap in theoretical understanding and modeling capability exists in the warm dense matter regime which is intermediate between condensed matter and plasma phases. This regime ranges from solids with temperatures comparable to the Fermi energy to materials which are plasma-like but too dense and/or cold to permit description by any standard solutions of plasma physics. A theoretical understanding of the warm dense phase of matter is critically important for progress in a variety of scientific endeavors including strongly-coupled plasma physics, high temperature condensed matter physics, inertial fusion, and astrophysics. Femtosecond optical excitation of solids is a unique means for studying the transition from a cold, dense solid to a hot plasma. Time-dependent x-ray absorption data are critical for testing computational models and for advancing our theoretical understanding of material physics in this regime. In addition, theoretical work in this area at Lawrence Livermore National Laboratory will directly support a broad range of experimental activities at the UXS facility by providing advanced modeling capabilities for interpreting x-ray spectral measurements, particularly in the complicated near-edge region.

\section{Solid-Solid Structural Changes and Phase Transitions}

Transitions from one distinct solid phase or crystallographic structure to another represent another class of phase transitions in which the underlying dynamics are determined by atomic motion on the time scale of a vibrational period. In extended solids, transformations of this type are difficult to study at the fundamental level since they nucleate at defect sites and evolve macroscopically via the creation of new defects and nucleation sites at the interface region. Semiconductor nanocrystals are a unique model system for investigating the structural dynamics of solid/solid phase transition since in this quantum size regime the transition begins to behave as a molecular isomerization. Static x-ray measurements have demonstrated a completely reversible, pressure-induced transition between wurtzite and rocksalt structure in these materials. By initiating the phase transition with a femtosecond optical pulse, the structural evolution can then be probed via time-resolved EXAFS or x-ray diffraction techniques.

Ultrafast x-ray measurements can also be applied to understand the dynamical properties of "complex" materials in which numerous degrees of freedom (such as charge, spin, and lattice) strongly interact. Time-resolved diffraction and EXAFS measurements can provide direct information about the impulsive response of the lattice to rapid carrier excitation and charge redistribution. In recent experiments, the polaron dynamics in manganites exhibiting colossal magnetoresistance have been studied via time-resolved Bragg diffraction.

\section{Ultrafast Photochemical Reactions}

In chemical reactions, the scientific challenge is to understand the structural evolution of the "transition state" intermediate between reactant and product species. The making and breaking of chemical bonds and the rearrangement of atoms in this regime ultimately determines which reactive pathways are favored. Advancing our understanding of condensed-phase reactions requires direct structural measurements on the fundamental time scale of a vibrational period. EXAFS and XANES will be applied on the femtosecond time scale to probe bond 
lengths, coordination, and bonding geometries during the course of reactions. Initial experiments will focus on spin-crossover reactions in Fe based organo-metallic molecules. In this system, the normally forbidden spin transition is thought to be facilitated by changes in the metal-ligand bond distance (which in turn modifies the spin-orbit coupling) on the sub-picosecond time scale.

A wealth of important chemistry occurs in solution, where the influence of the solvent environment is significant, and yet poorly understood. Experiments will apply time-resolved EXAFS and XANES to simple diatomic model systems (e.g. $\mathrm{I}_{2}$ ) to studying the effects of solvent caging and solvent/solute coupling and how they influence the photodissociation process. Subsequent experiments will extend this work to the more complicated reaction dynamics of solvated tri-atomic systems (e.g. $\mathrm{I}_{3}{ }^{-}$and $\mathrm{ClO}_{2}$ ). In addition to solute dynamics, ultrafast x-ray measurements can provide direct information about the transient structural response of the local solvent environment in response to rapid changes in the solute charge state. These fundamental questions of structural dynamics also apply to chemical reactions in a protein environment, which are the basic structural events that drive biological processes.

\section{Atomic and Molecular Physics with Femtosecond Optical and X-ray Pulses}

The availability of femtosecond EUV and x-ray pulses in combination with ultrashort optical pulses will open new areas of research in AMO physics by simultaneously providing core level photon energies for atomic specificity and ultrafast time resolution. These tools will enable the characterization of energy levels, bonding, and photoionization as a function of the evolving molecular geometry. For example, XANES will probe the dependence of the near-edge absorption resonances on bond distances and angles in molecules in which coherent vibrational wavepackets have been excited along a normal coordinate of interest. Time-resolved photoelectron spectroscopy will probe the evolution of molecular orbitals and their degree of localization as a function of bond distance in molecules during photoexcitation and photodissociation. Complementary photoelectron spectroscopy experiments will probe the core levels of molecules oscillating periodically between neutral and "ion-like" states due to the coherent motion of a an electronic wavepacket of excited Rydberg levels.

The UXS facility also provides a unique opportunity to develop a new area of high-field AMO research. High-energy x-ray photons can be used to probe atomic and molecular systems which are simultaneously subjected to field strengths which are comparable to the nuclear Coulomb fields. Such research requires both femtosecond duration optical pulses to create the high-field conditions, and x-ray probe pulses of comparable duration to characterize the coupled atom-field (or molecule-field) system that exists only while the high field is applied. X-ray absorption and/or photoemission measurements from a laser-dressed atom can provide direct information on the energy-level structure and distribution of population within the light-matter system. High-field AMO studies of the dressed continuum and bound-state energy level structure will provide a foundation for understanding high-intensity matter-field interactions in more complex systems such as molecules and condensed matter. 


\section{Relation to U.S. Department of Energy Programs}

The UXS facility is driven by the need to understand the behavior of matter at the atomic level within the fundamental (femtosecond) time scale in which structure evolves via the making and breaking of chemical bonds and the rearrangement of atoms. Understanding matter at the fundamental level is one of the scientific drivers identified in the Strategic Plan of the Office of Science. The UXS facility supports specific existing BES programs within the Division of Materials Sciences, as well as the Division of Chemical Sciences. These include programs in ultrafast dynamics in condensed matter, nanostructure materials, chemical dynamics, and AMO physics. The UXS facility is also in support of current DOE Defense Programs research in highenergy-density physics. This proposal is consistent with the DOE mission to provide national user facilities and research instrumentation that is beyond the capability and scale of university laboratories. Finally, the UXS facility will play a critical role in developing a new scientific field (ultrafast $\mathrm{x}$-ray science) and will serve as a proving ground for experiments that will in the future take advantage of $4^{\text {th }}$ generation source providing ultrashort $\mathrm{x}$-ray pulses.

\section{Organization and Management}

The UXS facility will operate in the spirit of a collaborative research center. Because ultrafast x-ray science is an emerging field, it is important that scientific applications and experiments be developed in synergy with new instrumentation and measurement techniques. Experiments will be conducted in collaborative teams comprised of researchers with complementary scientific and technical expertise. The scientific development of the UXS facility will be guided by a Beamline Steering Committee which is representative of the core research team, with membership rotating on an annual basis. The committee will be a vehicle for determining experimental priorities (allocation of beamtime) only within the core research group and will make decisions related to the operation of the facility. Beam time for other UXS facility users (outside the core research group) will be allocated according to the existing peer-reviewed Independent Investigator Program at the ALS. The steering committee will be co-chaired by R.W. Falcone (U.C. Berkeley-Physics) and R.W. Schoenlein (LBNL-Materials Sciences).

The technical design and construction of the UXS facility will be supervised by a project manager selected by the Beamline Steering Committee together with ALS management. Experienced personnel for managing the major components of this project have already been identified: superconducting undulator: R. Schlueter (LBNL Engineering), beamline and optics: P. Heimann (LBNL ALS Experimental Systems Group), modifications to the ALS storage-ring lattice D. Robin (LBNL ALS Accelerator Physics Group), femtosecond laser system R. Schoenlein (LBNL Materials Sciences Division).

\section{Budget}

Table 1 provides a summary of the construction costs for the UXS facility over the projected three-year course of the project. Separate labor and equipment costs are indicated for each year of the project, and for each of the major components of the project. LBNL overhead and contingency is included and is distributed proportionally for each table entry. 


\begin{tabular}{|l|r|r|r|r|r|r|r|}
\hline & $\begin{array}{c}\text { Labor } \\
2002\end{array}$ & $\begin{array}{c}\text { Equipment } \\
2002\end{array}$ & $\begin{array}{c}\text { Labor } \\
2003\end{array}$ & $\begin{array}{c}\text { Equipment } \\
2003\end{array}$ & $\begin{array}{c}\text { Labor } \\
2004\end{array}$ & $\begin{array}{c}\text { Equipment } \\
2004\end{array}$ & $\begin{array}{c}\text { System } \\
\text { Totals }\end{array}$ \\
\hline Undulator & 0 & 0 & 390 & 230 & 305 & 280 & 1,205 \\
\hline Front End & 0 & 0 & 172 & 335 & 0 & 0 & 507 \\
\hline Beamline & 135 & 640 & 435 & 275 & 300 & 350 & 2,135 \\
\hline Endstations & 0 & 0 & 0 & 437 & 0 & 0 & 437 \\
\hline Laser System & 123 & 1225 & 154 & 782 & 31 & 25 & 2,340 \\
\hline $\begin{array}{l}\text { Project } \\
\text { Management }\end{array}$ & 52 & 0 & 104 & 0 & 78 & 0 & 234 \\
\hline Escalation & 0 & 0 & 46 & 41 & 27 & 14 & 128 \\
\hline Yearly Totals & \multicolumn{2}{|c|}{2,175} & \multicolumn{7}{|c|}{3,401} & \multicolumn{7}{|c|}{1,410} & 6,986 \\
\hline
\end{tabular}

Table 1. Ultrafast X-ray Science facility construction costs over 3 year period in $\mathrm{K} \$$. LBNL overhead and contingency are included. Escalation rates are included at $2.0 \%$ per year for equipment and $3.7 \%$ per year for labor 


\section{Ultrafast X-ray Science Facility at the Advanced Light Source}

\section{Introduction and Background}

We propose to develop a unique facility for ultrafast $\mathrm{x}$-ray research. The proposal is motivated by the scientific need to understand the behavior of matter at the fundamental level. That is, understanding the structure of matter at the atomic scale, within the time period in which that structure evolves via the making and breaking of chemical bonds and the rearrangement of atoms. The relevant time interval for such dynamics is a vibrational period, which is on the order of $100 \mathrm{fs}$. Over the past 25 years, x-ray techniques applied with modern synchrotron sources have driven rapid advances in our understanding of the static or time-averaged structure of condensed matter on the atomic scale. Now, with the development of ultrashort pulse x-ray sources, time-resolved x-ray science is rapidly emerging in order to investigate structural dynamics and atomic motion occurring on time scales from milliseconds to femtoseconds. The development of this interdisciplinary research field will address important scientific questions in physics, chemistry, materials science, and biology.

For physical chemists, the direct observation of the molecular structure of "transitionstates" (intermediate conformations between reactant and product species) has been a major goal since Eyring and Polanyi formulated "transition-state theory" in the 1930's. Even at that time, they recognized that the transition state is extremely short-lived, as dictated by the time scale of an atomic vibrational period, $\sim 100$ fs. Indeed, the period of a vibrational oscillation is fundamental not only for molecular systems, but for condensed matter in general (even crystalline solids) because this is the limiting time scale on which structural dynamics occur. The motion and rearrangement of atoms on the time scale of a vibrational period ultimately determine the course of structural phase transitions in solids, the kinetic pathways of chemical reactions, and even the function and efficiency of biological processes.

The first spectroscopic measurements on the femtosecond time scale were made possible by the successful modelocking of the cw dye laser nearly three decades ago [1]. Because of the subsequent revolution in ultrafast laser technology, measurements of transient dynamics with a resolution of better than $10 \mathrm{fs}$ (over a spectral range from the ultraviolet to the infrared) are now routine [2]. The scientific significance of applying femtosecond optical pulses to probe the transient structure of transition states on the fundamental time scale in which they exist, was specifically recognized in the award of the 1999 Nobel Prize in Chemistry to A.H. Zewail. While femtosecond lasers afford access to this time regime, they suffer from the limitation that visible photons probe only the optical properties of condensed matter systems. Optical properties in condensed matter are a function of electronic states extending over multiple atoms. Therefore, they are only indirectly related to the underlying atomic structure. Extracting quantitative structural information from optical properties is essentially impossible for most systems. 
The development of femtosecond x-ray science portends another scientific revolution by combining the ultrafast temporal resolution of femtosecond pulses and stroboscopic pump-probe techniques with the structural specificity of $\mathrm{x}$-ray photons. X-rays are ideal probes of atomic structure because they interact with core electronic levels that are closely bound to the atomic nucleus. A growing number of research groups around the world are applying ultrafast $\mathrm{x}$-ray techniques to investigate structural dynamics in a variety of condensed matter systems [3-22], however, future progress in this field is substantially limited at present due to the lack of suitable short-pulse x-ray sources.

A number of important advances have been made in the generation of femtosecond x-ray pulses using a variety of techniques including: high-order harmonic generation from femtosecond optical pulses [23-26], laser-plasma based x-ray sources [11,18,27], laser-driven $\mathrm{x}$-ray diodes [17], and femtosecond $\mathrm{x}$-rays from Thomson scattering [15]. While initial timeresolved x-ray experiments have been performed using these sources, they suffer from significant limitations in one or more critical parameters including: (a) $x$-ray average brightness and/or flux, (b) x-ray photon energy, tunability, and spectral range, and (c) x-ray pulse duration and control.

While modern synchrotrons have revolutionized $\mathrm{x}$-ray science by providing highbrightness, tunable x-ray beams, a significant limitation of such sources for time-resolved measurements is the pulse duration (typically greater than $30 \mathrm{ps)}$ as determined by the duration of the stored electron bunch. The Department of Energy, Basic Energy Sciences Advisory Committee (BESAC) report on Novel Coherent Light Sources [28] recognizes that an important new capability of proposed $4^{\text {th }}$ generation $\mathrm{x}$-ray sources is ultrashort pulses for time-resolved structural studies. Ideally such a $4^{\text {th }}$ generation source would provide the flux, brightness and tunability $(0.1-20 \mathrm{keV})$ which is currently available from $3^{\text {rd }}$ generation synchrotrons, with a time resolution of $100 \mathrm{fs}$ or better, at moderate $(\mathrm{kHz})$ repetition rates. The BESAC report further recommends "better utilization of existing $3^{\text {rd }}$ generation sources and table-top lasers as proving grounds for innovative science and experiments planned for light sources of the future." [28]

We propose a synchrotron-based user beamline for ultrafast x-ray research. This proposal stems from long-standing research efforts in ultrafast $\mathrm{x}$-ray spectroscopy and the development of femtosecond x-ray sources and techniques at both U.C. Berkeley (in the Falcone group) $[3,13,16,18]$ and at Lawrence Berkeley National Laboratory [4,9,15,29-31] (funded through Laboratory Directed Research and Development). In particular, the Ultrafast X-ray Science (UXS) facility takes maximum advantage of the unique capabilities of a $3^{\text {rd }}$ generation synchrotron, and will provide $200 \mathrm{fs}$ x-ray pulses with an average flux $\left(\sim 10^{8} \mathrm{ph} / \mathrm{s} / 0.1 \% \mathrm{BW}\right)$ and average brightness $\left(\sim 10^{11} \mathrm{ph} / \mathrm{s} / \mathrm{mm}^{2} / \mathrm{mrad}^{2} / 0.1 \% \mathrm{BW}\right)$ which are many orders of magnitude beyond what is presently available in the sub-picosecond time regime (see section VII.2). The facility is based on a recently-demonstrated technique for generating femtosecond x-ray pulses from a synchrotron storage ring [4,29] in which the energy of an ultrashort slice of the stored electron bunch is modulated using a femtosecond optical pulse interacting with the electron beam in a wiggler [30]. The characteristics of the Advanced Light Source - particularly the low beam energy and low emittance in addition to the expertise at the Berkeley Lab and U.C. Berkeley, make this laboratory best positioned to develop such a facility for ultrafast x-ray research. Furthermore, a unique opportunity presently exists at the ALS because the sole 
available straight section is fortuitously located immediately adjacent to the existing wiggler which is required for laser-modulation of the electron beam. Finally, the UXS facility will make use of a state-of-the-art high-average-power femtosecond laser system. Critical technology for this laser system is now available from commercial vendors.

The unique capabilities of this facility will be instrumental in advancing the field of timeresolved $\mathrm{x}$-ray science. The facility is being proposed by a core research team comprised of leading research groups in ultrafast $\mathrm{x}$-ray science and includes complementary expertise in $\mathrm{x}$-ray spectroscopy using synchrotron beamlines, ultrafast optical spectroscopy, beam physics, laser/electron beam interactions, and femtosecond lasers. In the near term, this facility will provide a proving ground for the ultrafast $\mathrm{x}$-ray science and measurement techniques that may ultimately be done at a $4^{\text {th }}$ generation $\mathrm{x}$-ray source providing ultrashort $\mathrm{x}$-ray pulses. In the longterm, the high-repetition rate femtosecond x-ray pulses provided by this facility will be an important complement to a future x-ray FEL which is expected to provide substantially higher peak x-ray flux at lower repetition rates, suitable for single-shot experiments.

The development of this facility is consistent with the recommendations of the BESAC Panel on Novel Coherent Light Sources (S.R. Leone - chair), and has been endorsed by the ALS Scientific Advisory Committee, which has reserved the ALS straight section 6 for this project. This project was favorably reviewed by the LBNL Director's Review Committee for the ALS, and was enthusiastically endorsed by the BESAC Sub-Panel (Y. Petroff - chair) charged with reviewing the Advanced Light Source for the Department of Energy:

\begin{abstract}
Perhaps the most innovative, unique capability being developed at the ALS is the femtosecond spectroscopy and diffraction work discussed by R. Falcone and R.W. Schoenlein. For the study of ultrafast chemical reactions, phase transition, surface dynamics and a wide variety of critical biological processes operating on the sub-picosecond time scale, direct $x$-ray experiments have long been a dream. Impressive first steps along these lines have now been done at the ALS using femtosecond laser excitation of the sample, monitoring the evolving phenomena by $x$-ray diffraction with a streak camera ( $R$. Falcone et al.). As this capability is advanced there will clearly be many interesting measurements that will be enabled. By far the most intriguing, however, are the successes reported by R.W. Schoenlein et al. in production of $100 \mathrm{fs}$ $x$-rays by scattering of a femtosecond laser off of the electron beam packet in the synchrotron itself (femtosecond slicing). Used in pump/probe schemes with another femtosecond laser on the sample there are a vast number of experiments that will be possible for the first time. In this development the ALS is poised to be the world leader for many years to come. The panel feels that the development of the new undulator beamline for femtosecond $x$-ray science now being discussed should receive top priority. There is no comparable effort in any other synchrotron light source in the world (except for Free Electron Laser Projects, that will take much more time).
\end{abstract}

(from the BESAC Sub-Panel Report on the Advanced Light Source, February 2000 [28])

\title{
II. Objectives
}

A unique opportunity exists for the development of a dedicated beamline at the ALS that will fill a critical need for the growing ultrafast x-ray research community. We request DOE funding for the design and construction of this ultrafast $\mathrm{x}$-ray science facility which consists of four main parts: (1) a superconducting undulator source which will provide the highest possible photon flux and brightness in the $0.2-10 \mathrm{keV}$ range, (2) an $\mathrm{x}$-ray beamline consisting of imaging optics to provide a tunable monochromatic x-ray beam in a micro-focus spot, (3) a high- 
repetition rate femtosecond laser system and associated beam delivery optics which will serve the dual purposes of providing laser pulses for slicing the synchrotron beam (for generation of femtosecond X-rays) as well as tunable "pump" pulses for sample excitation, and (4) endstation components including an ultrafast streak-camera for measurements on picosecond time scales, a UHV vacuum chamber with a photoelectron spectrometer, and gated detectors ( 2 nsec gate width) for isolating individual synchrotron pulses. Substantial in-kind equipment support is provided by the existing (protein crystallography) wiggler which the UXS facility will use (simultaneously with operation of the crystallography beamline) for laser-modulation (slicing) of the stored electron beam on the femtosecond time scale.

\section{Ultrafast X-ray Science Facility Overview}

The UXS facility will be implemented as a general user facility accessible through the Independent Investigator Program at the ALS, with preferential access provided to the core research team (see VIII.2 for proposed allocation of beam time). The facility will be dedicated to time-resolved $\mathrm{x}$-ray measurements with temporal resolution ranging from 200 fs to $\sim 2 \mathrm{nsec}$. The highest possible flux and brightness in the $0.2-10 \mathrm{keV}$ range will be generated using a superconducting undulator in ALS straight sector 6. For the highest time-resolution measurements, the facility will provide $200 \mathrm{fs}$ x-ray pulses (at $40 \mathrm{kHz}$ repetition rate) generated via laser-modulation of the electron beam energy in the existing protein-crystallography wiggler (W16). Operation of the wiggler at the smallest gap will allow simultaneous use of the wiggler by the protein-crystallography beamline (BL5.0) and by the UXS facility. Furthermore, the present wiggler location (immediately upstream from the proposed undulator) is optimum for generating the shortest $\mathrm{x}$-ray pulses. Measurements on the picosecond time scale (at $40 \mathrm{kHz}$ repetition) will make use of the nominal $30 \mathrm{ps}$ x-ray pulses from the ALS in combination a femtosecond laser system synchronized to the storage ring. In addition, an ultrafast x-ray streak camera may be used in combination with the laser system to achieve a temporal resolution of 1 ps. The full flux and brightness (at $500 \mathrm{MHz}$ repetition rate) of this beamline will be available for static structural measurements and for dynamics on the nanosecond time scale.

The beamline will consist of a 1:1 imaging optic suitable for both soft $\mathrm{x}$-rays and hard X-rays. A double-crystal monochromator will provide a resolution of $\Delta \lambda / \lambda \sim 1 / 3000$ over the spectral range from 2-10 keV. A complementary, interchangeable plane-grating monochromator will provide a resolution of $\Delta \lambda / \lambda \sim 1 / 2000$ from 0.2 to $2 \mathrm{keV}$, and will additionally provide the capability for dispersive measurements over a spectral bandwidth of $\sim 100 \mathrm{eV}$. A mechanical chopper operating at $40 \mathrm{kHz}$ will allow selection of a $200 \mathrm{nsec}$ window of the $\mathrm{x}$-ray pulse train, synchronous with the laser system. An x-ray focal spot of $100 \mu \mathrm{m}$ (horizontal) by $70 \mu \mathrm{m}$ (vertical) will be delivered to the sample area by grazing-incidence focusing optics operating over the entire spectral range of the beamline.

The UXS facility will include a high-average-power femtosecond laser system based on chirped-pulse amplification in $\mathrm{Ti}: \mathrm{Al}_{2} \mathrm{O}_{3}$, and synchronized to the storage ring with $\sim 1$ ps accuracy. The laser system will provide two amplified pulse trains (from a single mode-locked oscillator) at a repetition rate of $40 \mathrm{kHz}$. One pulse train will provide for energy-modulation (slicing) of the electron beam in wiggler W16, and the second will provide optical pulses for sample excitation. The laser system will include an optical parametric amplifier for generating 
excitation pulses over the largest possible spectral range. The endstation hutch will accommodate a variety of x-ray measurement techniques, and will include two UHV sample chambers, an ultrafast x-ray streak camera, appropriate gated detectors for use in x-ray diffraction, EXAFS, and XANES measurements, as well as a hemispherical analyzer for photoemission measurements.

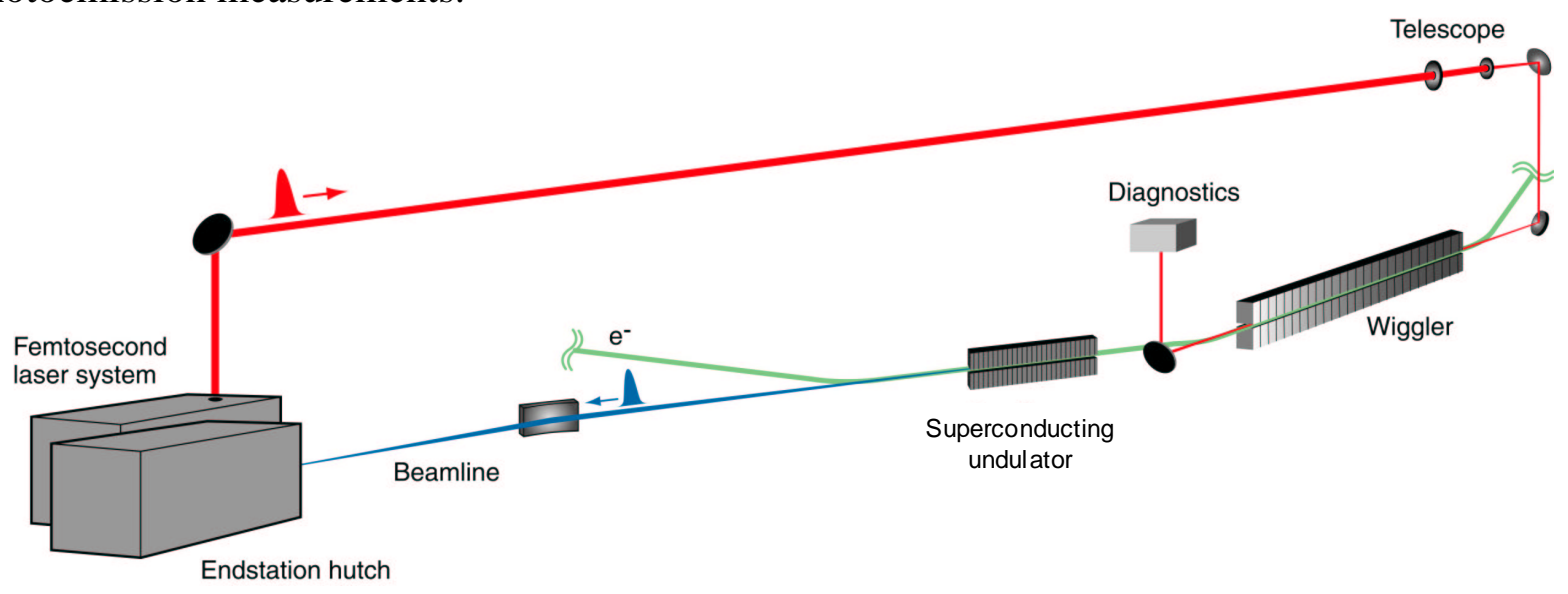

Figure 1. Schematic illustration of the proposed UXS facility including existing wiggler (for laser modulation of the electron beam), superconducting undulator, beamline, endstation hutch, and femtosecond laser system.

\section{Scientific Program}

\section{IV.1. Introduction}

Structural dynamics in condensed matter are ultimately governed by the motion and rearrangement of atoms on the fundamental time scale of a vibrational period. The integration of $\mathrm{x}$-ray measurement techniques, a high-brightness femtosecond $\mathrm{x}$-ray source, femtosecond lasers, and stroboscopic pump-probe techniques will provide the unique capability to address fundamental scientific questions in solid-state physics, chemistry, AMO physics, and biology involving structural dynamics. Thus, the scientific research program at the UXS facility will be interdisciplinary in nature and cross-cutting in scope. Furthermore, because the field of ultrafast $\mathrm{x}$-ray science is in its infancy, scientific applications and experiments will develop synergistically with advances in time-resolved $\mathrm{x}$-ray measurement techniques. Active collaboration between scientists with complementary expertise is critical to this development, and the scientific program at the UXS facility will actively promote such collaboration.
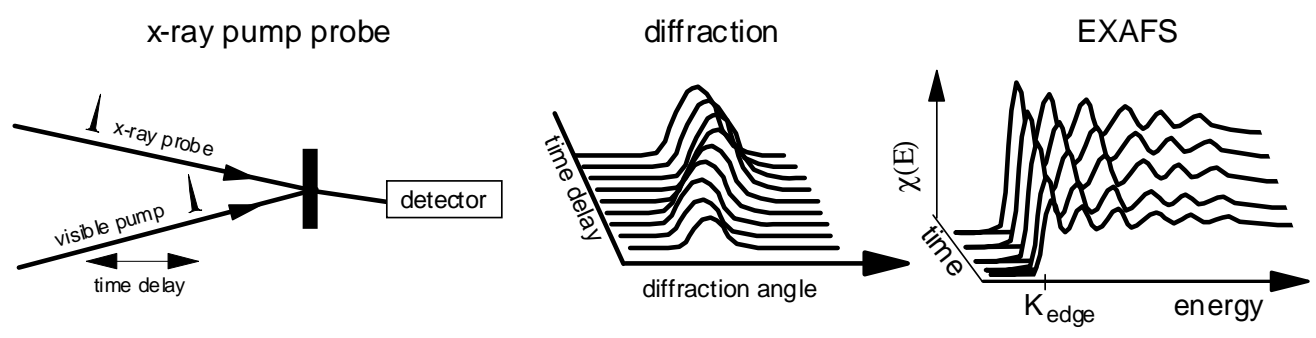

Figure 2. Schematic of time-resolved x-ray diffraction and EXAFS using femtosecond x-ray pulses and stroboscopic pump-probe measurement techniques. 
The UXS facility will provide for time-resolved implementations of numerous x-ray techniques including: Bragg diffraction; Laue diffraction; extended $\mathrm{x}$-ray absorption fine structure (EXAFS), measured via transmission, fluorescence, and photoelectron yield; x-ray absorption near-edge structure (XANES); and x-ray photoelectron spectroscopy (XPS). Samples will include crystalline and amorphous solids, surfaces, nanostructures, liquid solutions and gases. The description of the scientific program is organized into five general research areas: (a) order/disorder transitions in solids and at surfaces; (b) structural dynamics in warm dense matter; (c) solid-solid structural transitions; (d) ultrafast chemical reaction dynamics; and (e) atomic and molecular physics with femtosecond optical and x-ray pulses.

\section{IV.2. Order-Disorder Transitions in Solids and at Surfaces}

The dynamics of phase transformations in solids are of fundamental scientific interest since they determine the genesis and evolution of new structural phases. The conventional description of simple first order solid/liquid phase transitions in crystals is based on a thermal model, which assumes local thermal equilibrium among the vibrational modes and between the electronic and vibrational energy reservoirs. In such a model, the phase transition proceeds via energy transfer to the lattice in the form of heat. The thermal model then predicts that following optical excitation, a phase transition will evolve on the time scale determined by electron-phonon energy exchange, typically several picoseconds. Implicit in such a description is the assumption that excitation or energy deposition to the solid is slow compared to a vibrational period or to electron-phonon scattering times. Indeed, time-resolved x-ray diffraction measurements confirm that annealing of silicon via excitation with nanosecond laser pulses follows a thermal melting model [20,32].

However, highly non-equilibrium conditions can be created in solids via femtosecond optical excitation. Ultrafast optical pulses can deposit energy on time scales that are short compared to electron-phonon interaction times; thereby creating electron temperatures well in excess of the underlying lattice temperature. Energy can even be deposited on time scales shorter than electron-electron scattering times ( $<100 \mathrm{fs}$ ), creating non-equilibrium (non-Fermi) electron distributions. Under such conditions, the thermal melting model breaks down, and new physical effects emerge.

This raises fundamental scientific questions about how phase transitions occur under nonequilibrium conditions, and specifically whether "melting" of a solid can occur on the time scale of a phonon period (i.e. before the vibrational modes reach thermal equilibrium above the melting temperature). Such a "plasma annealing" model was originally proposed more that 20 years ago, in an attempt to explain pulsed-laser annealing of Si [33,34]. Since then, theoretical models have predicted that in covalent crystals, the promotion of a large fraction of electrons from bonding to anti-bonding states will sharply reduce the restoring forces between atoms, rendering the crystal structure unstable on the time scale of $200 \mathrm{fs}$ [35-37]. Under such conditions, disordering may proceed in a manner analogous to photodissociation in molecules. Recent ab-initio molecular dynamics calculations for Si [38,39] predict that unique metastable structural phases, with coordination that is substantially different from either the solid or equilibrium liquid phase, can result from such non-equilibrium conditions [38,39]. 
To date, ultrafast non-equilibrium phase transitions in crystals with diamond and zincblende lattice structure ( $\mathrm{Si}, \mathrm{GaAs}$, and $\mathrm{InSb}$ ) have been studied using a variety of indirect optical techniques [40-46]. Recent optical experiments provide some evidence of laser-induced disordering in crystalline silicon on a time scale comparable to the phonon period ( $70 \mathrm{fs})[42,44,47]$. Nevertheless, because optical measurements do not provide direct information about the atomic positions and coordination, they are unable to address the fundamental scientific questions related to structural dynamics in this regime.

\section{Time-Resolved Bragg Diffraction of Order-Disorder Transitions}

Femtosecond $\mathrm{x}$-ray diffraction provides direct information about long-range atomic order (and loss of order) in crystalline systems and is a powerful tool for studying ultrafast phasetransitions and energy transfer in solids, especially metal and semiconductor material systems. A number of research groups around the world are beginning to apply time-resolved x-ray diffraction to investigate ultrafast phase transitions using a variety of short-pulse $\mathrm{x}$-ray sources $[3,5,10,11,16,27]$. Our early experiments in InSb made use of 300 fs X-ray pulses (at 30 $\mathrm{keV}$ ) generated via Thomson scattering between a terawatt laser pulse and $50 \mathrm{MeV}$ electrons from the ALS linac injector [15,48]. Time-resolved diffraction measurements made with this relatively low-flux source, shown in Fig. 3, clearly elucidate the energy transfer dynamics to the lattice from the initial electron-hole plasma created by the optical pulse. The process of electronphonon energy relaxation and the development of acoustic phonons from anharmonic optical phonons are manifest in the diffraction data as a distinct delay ( 10 ps) in the onset of lattice expansion following laser excitation [9]. The resulting strain (lattice expansion: $\Delta \mathrm{d} \sim 4 \mathrm{~m} \AA$ ) and strain propagation into the sample at the sound speed can be directly measured.

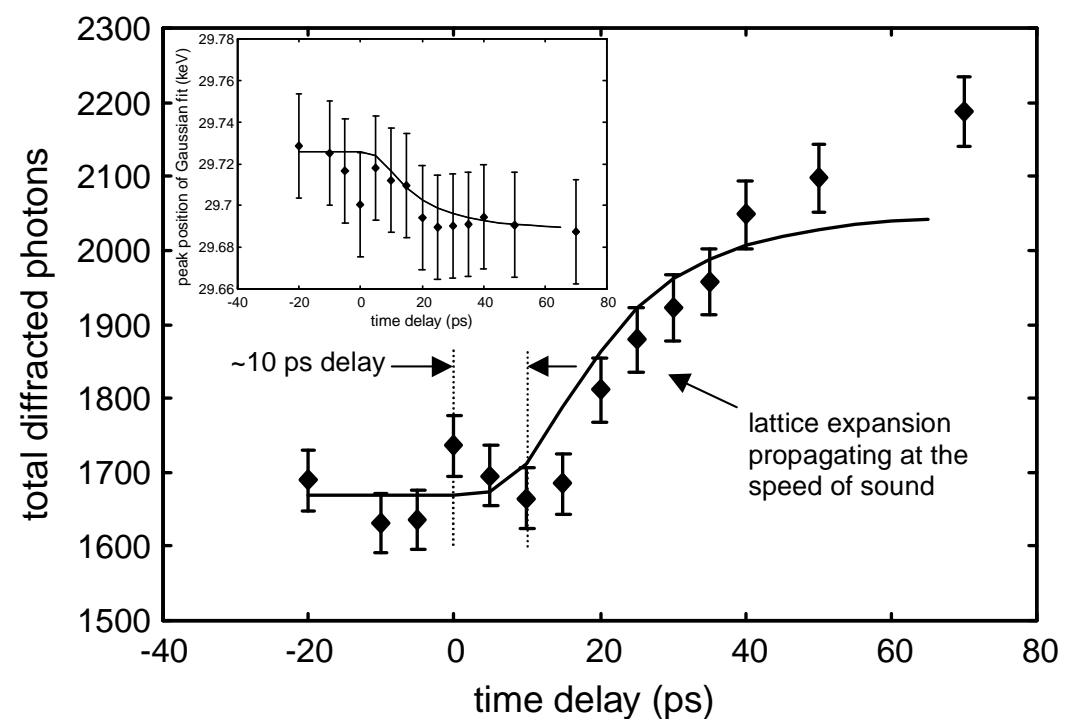

Figure 3. Diffracted $x$-ray photons $(30 \mathrm{keV})$, integrated over the InSb Bragg peak, as a function of time delay following laser pulse excitation. Inset: time-dependent shift in spectral position of Bragg peak. Solid lines are from a model calculation accounting for energy transfer from the electron-hole plasma to LO phonons to acoustic phonon population, and subsequent propagation of strain into the crystal. (Measured at the ALS Thomson-scattering source, from [9]). 
Investigation of non-thermal melting requires greater surface sensitivity since the highest electron-hole densities (which are thought to drive this process) are at maximum in a thin surface layer. Time-resolved diffraction measurements in InSb using $7.5 \mathrm{keV}$ x-rays (Fig. 4) show the first hint of non-thermal ultrafast disordering. (These measurements are more sensitive to surface layers due to the shorter attenuation length compared to $30 \mathrm{keV}$ photons). An overall drop in the diffraction efficiency is observed within 1 ps of excitation, indicative of the formation of a disordered surface layer $\sim 30 \AA$ thick [9]. Spectral measurements of the Bragg peak show a distinction between this overall loss in diffraction efficiency (at $1 \mathrm{ps}$ delay), and the strain propagation as evidenced by a broadening and shifting of the Bragg peak to lower energies (at 10 ps delay).
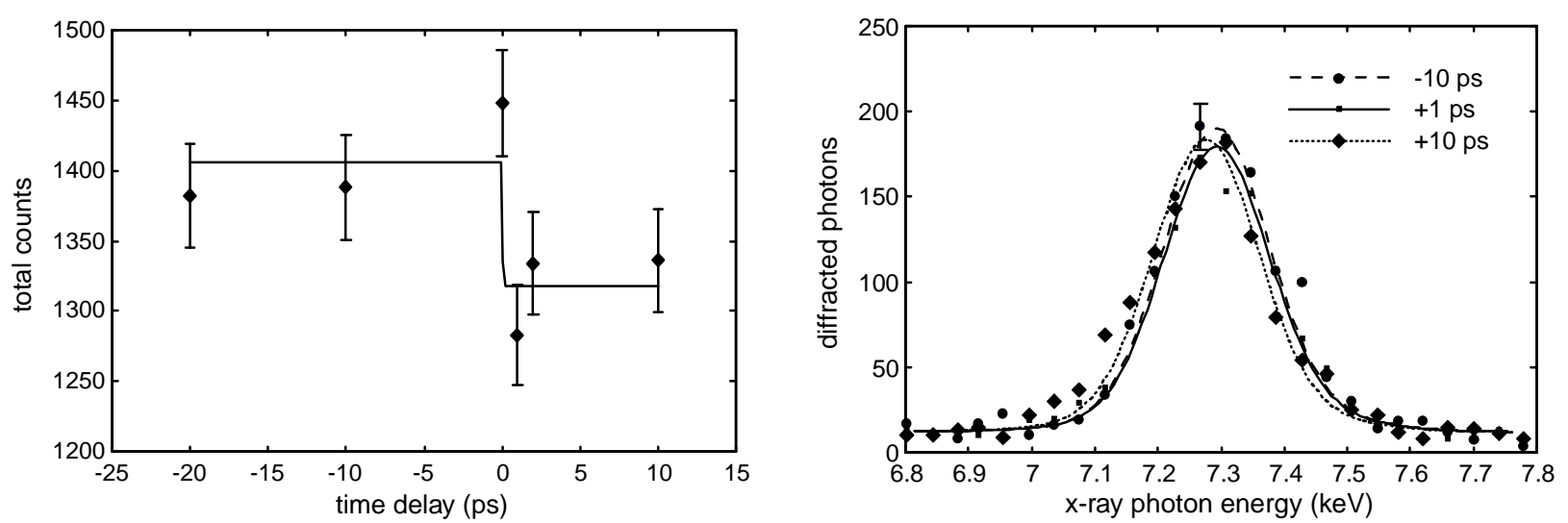

Figure 4. (left) Normalized integrated x-ray diffracted photons as a function of time delay. (right) Representative x-ray diffraction spectra (with simulated profiles) for different time delays, taken with 7.3 keV x-rays. (Measured at the ALS Thomson-scattering source, from [9]).

These experiments and more recent measurements using x-ray pulses generated from a laser produced plasma [10,11], have provided the strongest indication yet of non-thermal disordering driven directly by a high-density electron-hole plasma. They raise important scientific questions about the kinetics of disordering in this non-equilibrium regime as will be discussed later. At the same time, these measurements illustrate the severely limited capabilities of existing short-pulse x-ray sources. In particular, diffraction measurements require highbrightness $x$-ray sources because the angular acceptance of the Bragg rocking curve is on the order of only $100 \mu \mathrm{rad}$. This is in striking contrast with the $2 \pi$ divergence of laser-plasma sources and is a significant limitation even for the $\sim 10 \mathrm{mrad}$ divergence of the Thomsonscattering based source. Furthermore, the acceptance bandwidth $(\Delta \lambda / \lambda)$ of the Bragg rocking curve is $\sim 10^{-3}$ which is poorly matched by the $\sim 15 \%$ bandwidth of the Thomson source[15,48]. Finally, the enhanced x-ray brightness and temporal resolution provided by a synchrotron-based femtosecond $\mathrm{x}$-ray source will enable a quantitative interpretation of such ultrafast measurements.

Recently, the Falcone group (U.C. Berkeley) has developed a complementary approach for time-resolved diffraction measurements by applying an ultrafast $\mathrm{x}$-ray streak camera detector [13] at an ALS bend-magnet beamline. The flux and energy resolution (narrow bandwidth) provided by a synchrotron beamline enable the direct observation of energy-coupling from an optically-created electron-hole plasma to coherent vibrational modes of the lattice in 
InSb [3]. Figure 5 (left) shows the strong sinusoidal modulation of the x-ray diffraction intensity due to a coherent acoustic phonon mode. These measurements indicate that below a given threshold laser fluence, the primary mechanism of energy transfer between hot carriers and the lattice is coherent acoustic phonon generation via deformation potential coupling and LO phonon decay.
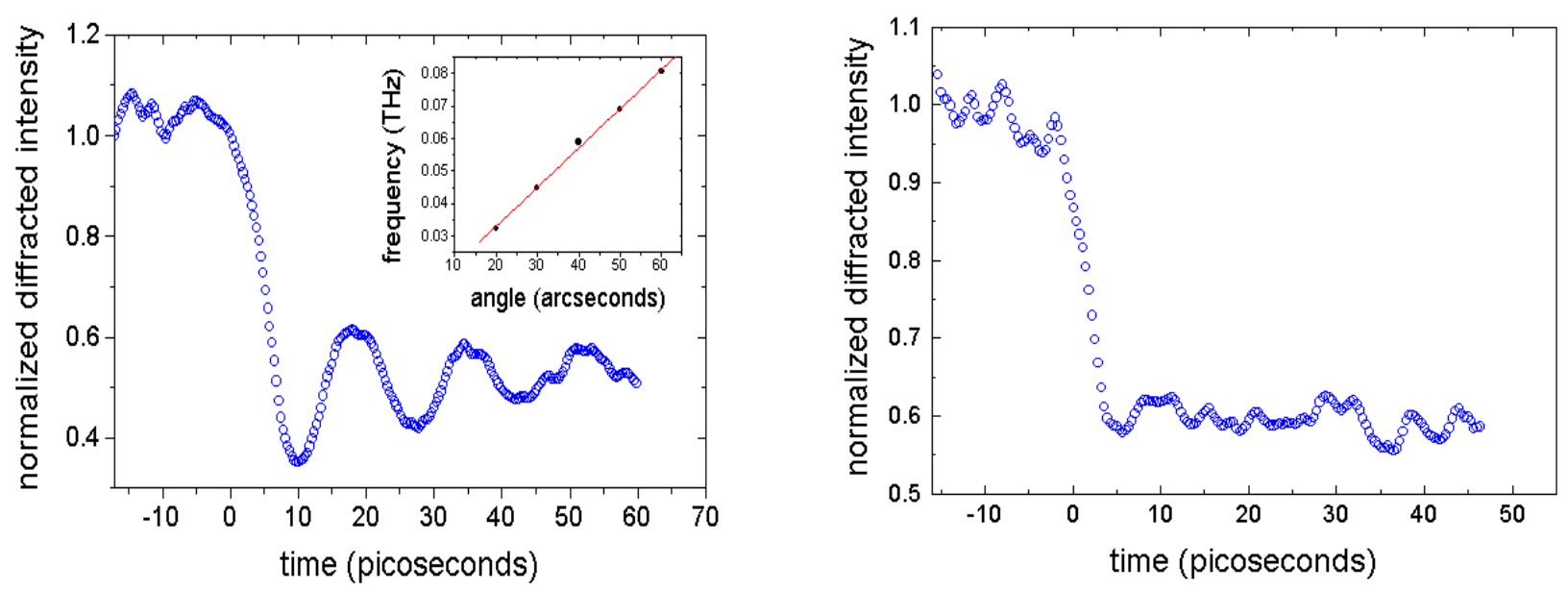

Figure 5. (left) Sinusoidal modulation of $\mathrm{x}$-ray diffraction by coherent acoustic phonons measured at 40 arcsec away from the (111) Bragg peak of InSb. The inset shows the phonon frequencies measured at different angular deviations from the Bragg peak. (right) At higher fluence, rapid disordering is indicated by the prompt drop in the diffraction efficiency and absence of vibrational coherence. (Measured at ALS beamline 7.3.3, from [3]).

The phonon dispersion relation (inset Fig. 5) is measured by sampling acoustic phonons of different momentum by changing the angle of the crystal. This new approach to doing phonon spectroscopy may be applied to a variety of solid-state systems, including ferroelectrics, high- $\mathrm{T}_{\mathrm{c}}$ superconductors, and other complex materials. At high laser-excitation levels, near the damage threshold of the material, strongly anharmonic or nonlinear states will be induced. These will manifest themselves in deviations from the harmonic phonon dispersion relations, which may be directly measured. In InSb, above a threshold in laser fluence (Fig. 5 right), it is found that the atoms no longer coherently oscillate around the equilibrium positions of the lattice. Instead they are driven into a disordered state where no atomic motion of long-range coherence (and thus no oscillation in the x-ray Bragg diffraction) can exist. This occurs on the time-scale of one-half of a phonon period, at which point the atomic displacement exceeds the Lindemann criterion of melting.

The generation of large amplitude coherent phonons is evidently an important step in non-thermal disordering of a crystalline lattice. Large-amplitude atomic motion may also lead to localization of normally extended vibrational states. However, present temporal resolution of $\mathrm{x}$-ray streak cameras, and the limited capabilities of femtosecond x-ray sources prevent a more complete study. Important questions remain about the time scale and mechanism for the order/disorder transition, the local structure of the disordered state, and the dynamics of the recrystallization. A better understand of these issues may have applications, for example, in laserprocessing of semiconductors. 


\section{Time-Resolved EXAFS of Order-Disorder Transitions}

Time-resolved Bragg diffraction measurements provide information about long-range order (and disorder), including strain propagation, and coherent phonon dynamics. Other x-ray techniques can be applied on the femtosecond time scale to elucidate the details of the local structural evolution from the solid to the liquid phase under non-equilibrium conditions created by pulsed laser excitation. Time-resolved EXAFS measurements can be applied to probe changes in short-range structure, bond lengths, and coordination, and thereby identify metastable structural phases that may be created. For example, recent $a b$ initio calculations by Silvestrelli et al. predict a transient metastable liquid phase in Si following heating by an ultrashort laser pulse [38,39]. Figure 6 illustrates the power of time-resolved EXAFS for distinguishing between crystalline silicon ( $\mathrm{Si}$, coordination: $4, \mathrm{r}=2.35 \AA$ ), conventional liquid-phase silicon ( $l$-Si, coordination: $6-7, r=2.4 \AA$ ), and the predicted metastable liquid phase with a high coordination more characteristic of liquid metals, $\left(l^{\prime}-\mathrm{Si}\right.$, coordination $\left.11-13, \mathrm{r} \cong 2.7 \AA\right)$.
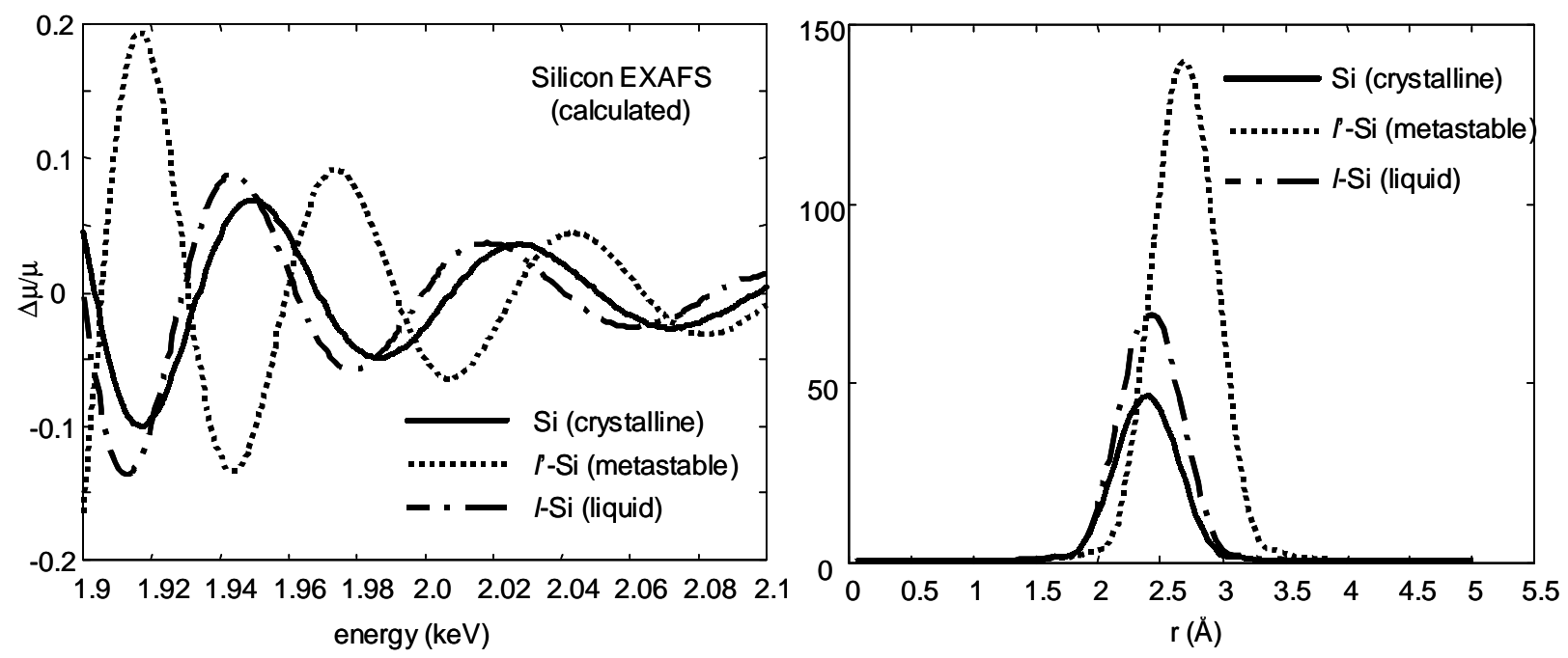

Figure 6. (left) Model EXAFS calculations and corresponding Fourier transforms (right) for crystalline $\mathrm{Si}(300 \mathrm{~K})$, liquid $\mathrm{Si}(1700 \mathrm{~K})$, and a predicted metastable liquid phase $(1700 \mathrm{~K})[38,39]$.

In addition, EXAFS measurements via photoelectron yield will provide the surface sensitivity (as mediated by the photoelectron escape depth, $\lesssim 100 \AA$ ) that is necessary to probe the region of highest electron-hole density within the optical absorption length. Furthermore, because EXAFS is sensitive to local order and coordination, and is element specific, measurements are not restricted to single-crystal samples. This opens the opportunity to investigate phase-transition dynamics in complex materials, nanostructures, and polycrystalline, materials in which material composition and/or domain boundaries may influence the dynamics.

It is known from experimental observation and verified by molecular-dynamics modeling that conventional melting is a heterogeneous, or nucleated process [49]. It proceeds from interfaces and grain boundaries due to the enhanced mobility of the surface atoms. Homogeneous melting, in which the entire crystal lattice becomes unstable to shear deformation, 
is usually observed only when surfaces are carefully passivated, and is manifest by a solid-liquid phase transition occurring at temperatures above the thermodynamic melting point (i.e. superheating) [49]. The non-equilibrium conditions created by femtosecond laser excitation can test the limits of the heterogeneous model of phase transitions. At sufficiently high carrier densities (with a significant fraction of electrons promoted from bonding to anti-bonding orbitals), the mobility of interior atoms should be largely unconstrained, and the solid-liquid phase transition may become a homogeneous process. Time-resolved EXAFS provides an opportunity to understand the behavior of materials in this unique regime by measuring the structural dynamics of solid-liquid phase transitions in polycrystalline materials. In the future, it may be possible to extend these techniques to study phase transitions in buried layers and interfaces as well.

Of particular interest are ultrafast phase transitions in nanostructure materials in which the nucleation model breaks down because the entire crystal is much smaller than a typical domain size. Equilibrium studies of semiconductor nanocrystals of CdS ( 200 $\AA$ diameter with nearly perfect crystalline structure) reveal that the melting temperature is strongly suppressed (by more than a factor of 2) with decreasing size, as a larger fraction of the constituent atoms are on the surface [50]. Essentially the entire nanocrystal starts to resemble a single defect, and the solid-liquid phase transition may occur homogeneously throughout an individual nanoparticle. By exciting a distribution of nanocrystals with a femtosecond optical pulse, the phase transition may be initiated simultaneously throughout the sample. The structural evolution during both disordering and re-crystallization may then be observed via time-resolved EXAFS at the Cd $\mathrm{L}_{\mathrm{I}}$-edge (at $\sim 4 \mathrm{keV}$ ). Preliminary optical-pump and optical-probe experiments on order-disorder phase transitions of $\mathrm{CdS}$ nanocrystals in solution are currently underway. Estimates based on simple equilibrium thermodynamics indicate that $\sim 100$ photons (at $\lambda=400 \mathrm{~nm}$ ) per nanocrystal should be sufficient to elevate the temperature to the melting point. Considering the nonequilibrium conditions created by excitation on the femtosecond time scale, this is likely an overestimate. Optical excitation will be at short wavelengths ( $>3 \mathrm{eV}$ photon energy) far from the exciton (homo-lumo) transition which is easily bleached.

\section{IV.3. Dynamics in Warm Dense Matter}

The construction of a tunable short-pulse x-ray source will provide an important step towards achieving a quantitative research capability into the warm dense matter (WDM) regime. The area of WDM refers to that part of the density-temperature phase space between solids and plasmas where the standard theories of condensed matter physics and/or plasma statistical physics are invalid. Thus, warm dense matter refers to states from liquid to greater than solid density with temperatures comparable to the Fermi energy. It also refers to those states of matter that are plasma-like, but are too dense and/or too cold to admit to standard solutions used in plasma physics. This is the region where plasmas become strongly coupled so that perturbation approaches fail as no small expansion parameters exist. Moreover it is the region where the temperature of a solid nears or exceeds the Fermi temperature, thus indicating the failure of standard cold condensed matter approaches. Warm dense matter is found in planetary interiors, cool dense stars, and in every plasma device which evolves from an initial solid phase (e.g., laser-solid matter produced plasmas as well as all inertial fusion schemes). 
To date, the study of dense plasmas has been severely hampered by the fact that laserbased probe methods are ineffective. The most useful diagnostic of local plasma conditions (the temperature, $T_{e}$, the density, $n_{e}$, and the ionization, $Z$ ) has been Thomson scattering using lasers. However, visible light will not propagate in systems in which the electron density exceeds $\sim 10^{22} \mathrm{~cm}^{-3}$. Thus, all dense matter can not be probed in depth by visible photons. Time-resolved $\mathrm{X}$-ray absorption experiments in the warm dense matter regime at the proposed UXS facility will provide a near-term solution to the problem of probing dense matter on sub-picosecond time scales. In this approach, warm-dense-matter $(10 \mathrm{eV})$ is created via laser excitation, and the evolution of the electronic and atomic structure can be probed via XANES and EXAFS. In the longer term, it is expected that $4^{\text {th }}$ generation X-ray sources will provide ultrashort X-ray pulses of sufficient intensity for creating warm dense matter (in addition to providing an ultrafast $\mathrm{x}$-ray probe capability).

The scientific interest in the warm dense matter regime arises because in dense plasmas the atoms and/or ions start to behave in a manner that is intrinsically coupled to the plasma. That is, the plasma starts to exhibit long- and short-range order due to the correlation effects between the atoms/ions. This intriguing regime, where the plasma can no longer be considered a thermal bath and the atoms are no longer well described by their individual properties, provides a tremendous challenge to researchers. For such a plasma the defining quantity is the coupling parameter $\Gamma$ which is the ratio of the inter-atomic potential energy to the thermal energy given by:

$$
\Gamma=\frac{Z^{2} e^{2}}{r_{0} k T} \text { where } r_{0}=\left(\frac{3 Z}{4 \pi n_{e}}\right)^{1 / 3}
$$

here $Z$ is the ion charge and $r_{o}$ is the inter-particle spacing. The regions of interest span the density-temperature phase space from modestly coupled $(\Gamma \leq 1)$ to strongly coupled $(\Gamma>1)$, while bridging the transition regimes between solid to liquid to plasma. In the limit of dense cool plasmas one arrives at the threshold of condensed matter. Here the perturbative approach is abandoned in favor of ground-state methods requiring complete renormalization of the atom/ion and its environment.

From a condensed matter perspective strong coupling is not the relevant measure, as the matter is highly structured and correlated. In approaching the warm dense matter regime from cold condensed matter, the issue becomes the temperature of the system relative to the Fermi energy, $E_{\text {Fermi }}$. In the case where $T<E_{\text {Fermi }}$ the normal methods of condensed matter theory will work as only a few valence/conduction bands need to be taken into account. However, when $T>E_{\text {Fermi }}$ the number of bands that are required to describe the system become enormous. In addition, one must also include both excited core states and ionized species in the description.

The region of the temperature-density plane (for $\mathrm{Al}$ ) where warm dense matter studies are important is illustrated in Fig. 7. The theoretical uncertainties are largest in the WDM region (indicated in blue) where the standard theoretical approaches fail and experiments are exceedingly difficult. The experimental study of WDM is difficult in part because the creation and isolation of matter in this regime is complicated. Although the plasma evolution of every density-pressure $(\rho-T)$ path that starts from the solid phase goes through this regime and plays an 
important role in its evolution, trying to temporally and spatially isolate warm dense matter remains a significant challenge.

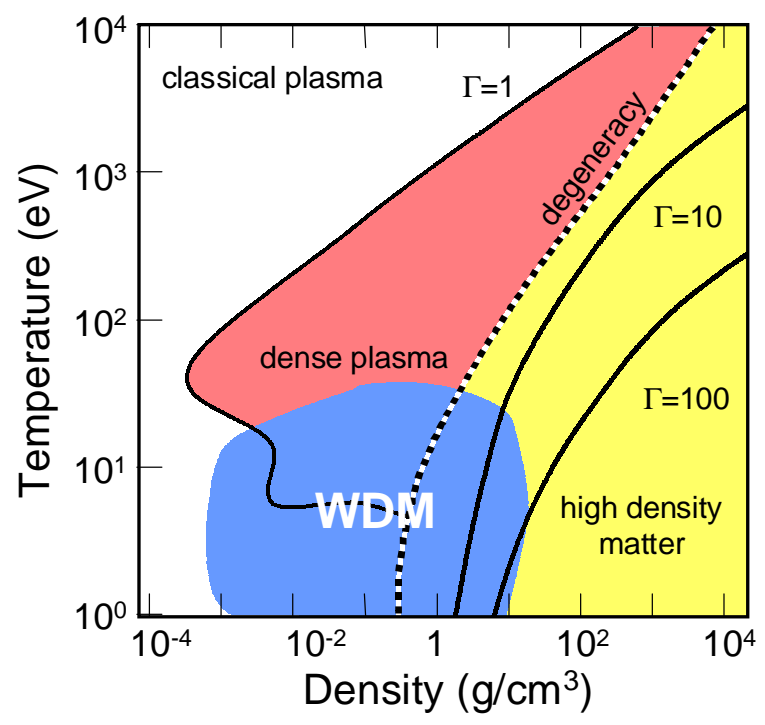

Figure 7. The temperature-density phase diagram for Al. The relevant regimes are noted, as are the various values of the coupling $\Gamma$. The region of greatest uncertainty is noted as WDM (warm dense matter).

The study of warm dense matter offers the possibility of exploring new frontiers in diverse areas including: strongly coupled plasma physics, high temperature condensed matter physics, and high pressure chemistry. Moreover, in many areas of research the transition from a cold dense solid to tenuous hot plasma occurs in a time-dependent manner, which is important for the physical description of the system and its evolution. Examples are numerous, with the most obvious being the $\rho-T$ track of inertial fusion targets, laser produced plasma sources, and shock heated systems.

There is also an astrophysical interest in warm dense matter. Large planets and low-mass stars exist in a state that can be described as warm dense matter. This is true whether the major constituents are hydrogen and helium or more complicated compounds. A correct description of a brown dwarf requires consideration of elements at Mbar pressures over a range of high densities and temperatures. Theories of formation of planetary bodies rely on structure models that are in turn determined by fundamentals like the equation of state (EOS) and constrained by observations. For example, the largest uncertainty in estimates of the structure of extra-solar planets is the EOS, which is the EOS of warm dense matter [51]. 
Three important factors are driving the development of WDM research and now enable significant progress to be made in this field:

- First, with a tunable femtosecond x-ray source one will be able, for the first time, to probe matter in the warm dense regime.

- Second, with the advance of theoretical methods, it is becoming possible to directly compare theory and experiment. New predictions will require verification by experimental data.

- Third, there are important mission oriented research requirements, e.g., inertial fusion and weapons related studies, that involve matter in the warm dense regime.

The objective of WDM experiments at the UXS facility is to obtain X-ray spectroscopic data that is necessary for accurately determining the equation of state for matter in the warm dense regime. The equation of state is a thermodynamic description of matter in terms of pressure, density and temperature, and how matter reacts to any change in one of those three variables. An improved understanding of this region is important because the fundamental physics that effect the EOS in the warm dense regime are precisely the physics that effect the macroscopic properties of matter. Indeed, the conductivity, the opacity, and other properties of warm dense matter all depend on similar levels of understanding. Therefore, the EOS data provides a test of our fundamental understanding of these systems.

The experiments proposed for the UXS facility will be conducted in close collaboration with the theoretical effort at Lawrence Livermore National Laboratory (LLNL). An initiative at LLNL, Creating a Quantitative Capability for the Warm Dense Matter Regime, is organized in a two-part parallel approach. Theoretical techniques that are usually applied to hot plasmas are being extended towards a regime that is colder and more strongly coupled. At the same time, condensed matter methods (such as ab initio quantum molecular dynamics) are being extended from cold solids $\left(\mathrm{T}<<\mathrm{E}_{\mathrm{Fermi}}\right)$ towards higher temperature solids.

At the UXS facility, experiments will be conducted on rapidly-heated thin film targets and the x-ray photoabsorption cross section will be measured as a function of wavelength and compared directly to theory. An energetic laser pulse can rapidly heat an initially cold sample of condensed matter to the warm dense regime. Short x-ray pulses can subsequently probe the sample, providing temporally resolved data related to the structure (via EXAFS), and electronic properties (via XANES), of the material. Experimental methods to probe warm dense matter at $3^{\text {rd }}$ generation light sources have been recently developed at the ALS. Preliminary experiments have been done at the using a bend magnet source (beamline 7.3.3) to measure the timedependent absorption spectrum of Si foils $(0.5 \mu \mathrm{m}$ thick) heated with a $10 \mathrm{~ns}$ laser pulse at an irradiance of $2 \mathrm{~J} / \mathrm{cm}^{2}$. Figure 8 shows the results of this initial experiment. The gray background is the reference spectrum (unheated) and the data show the sample absorption at $50 \mathrm{~ns}$ after laser heating. There is a $1.5 \mathrm{eV}$ shift of the Si K-edge toward lower photon energy that is attributed to the metallic character of the liquid silicon. Similar shifts have been seen recently in XANES spectra of $l$-Ge, which has been interpreted as an increase in the screening of the core hole in the metallic liquid phase [52]. 


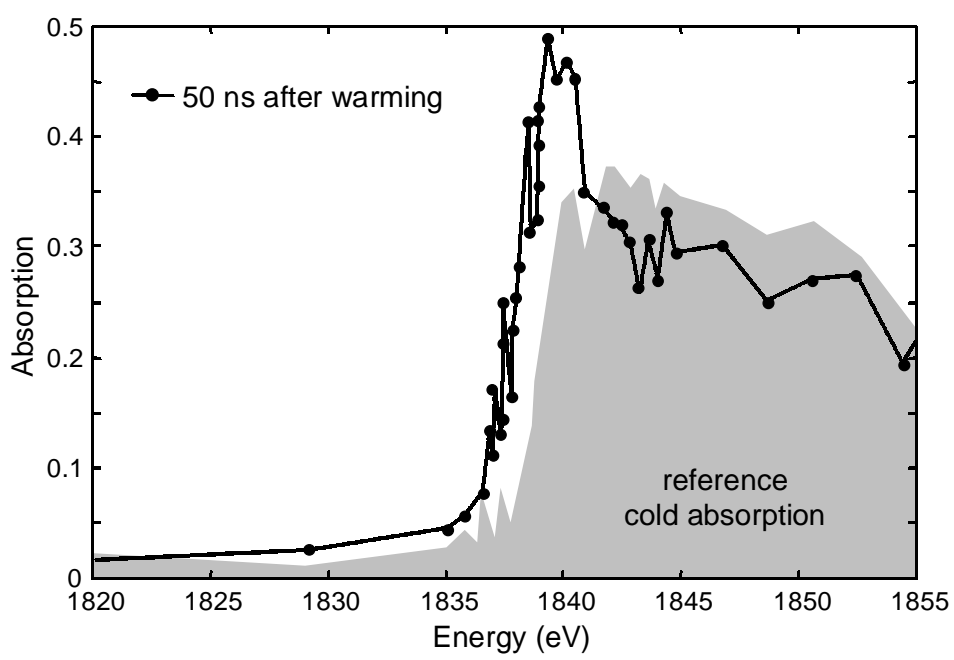

Figure 8. The first data on a warm dense sample using the ALS beamline 7.3.3 showing the x-ray absorption spectrum of a $0.5 \mu \mathrm{m}$ sample of $\mathrm{Si}$ in the spectral region around the K-edge. The gray area is the absorption spectrum of an unheated sample while the circles indicate the absorption at $50 \mathrm{~ns}$ after laser heating. Note the shift of the heated spectrum toward lower energy.

The second stage of experiments are now being implemented in anticipation of the experiments that will make use of a short pulse x-ray probe source from the proposed undulator beamline. Since the nature of the experiments requires that the sample be destroyed with each measurement, one needs to maximize the data acquisition rate by recording absorption spectra in a dispersive mode, thereby enhancing the signal by about two orders of magnitude. Furthermore, it is important to study the evolution of warm dense matter with minimum contamination from underdense material that is ablated from the target. This is achieved by using a short-pulse laser to heat the target rapidly. With these objectives in mind, experiments were recently initiated on ALS beamline 5.3.1 in which a short pulse laser $(<1 \mathrm{ps})$ was used to heat the sample and a variable-line-space grating coupled to a streak camera ( 2 ps resolution) was used to measure the entire absorption edge for each x-ray pulse. Absorption measurements of the Al L-edge (for a cold $1000 \AA$ foil) required only four shots, and indicate a vast improvement in data rate. Further, we use a thin foil to improve the uniformity of the sample that is being probed.

To illustrate the behavior of a thin Al foil irradiated by a short pulse laser we show in Fig. 9 the predicted temperature and density contours at several times after laser heating at an irradiance of $10^{13} \mathrm{~W} / \mathrm{cm}^{2}$. Note that the time scale on which changes occur is on the order of 200 fs. Further, we note that 1 ps after irradiation a relatively uniform temperature profile is established throughout the foil. 

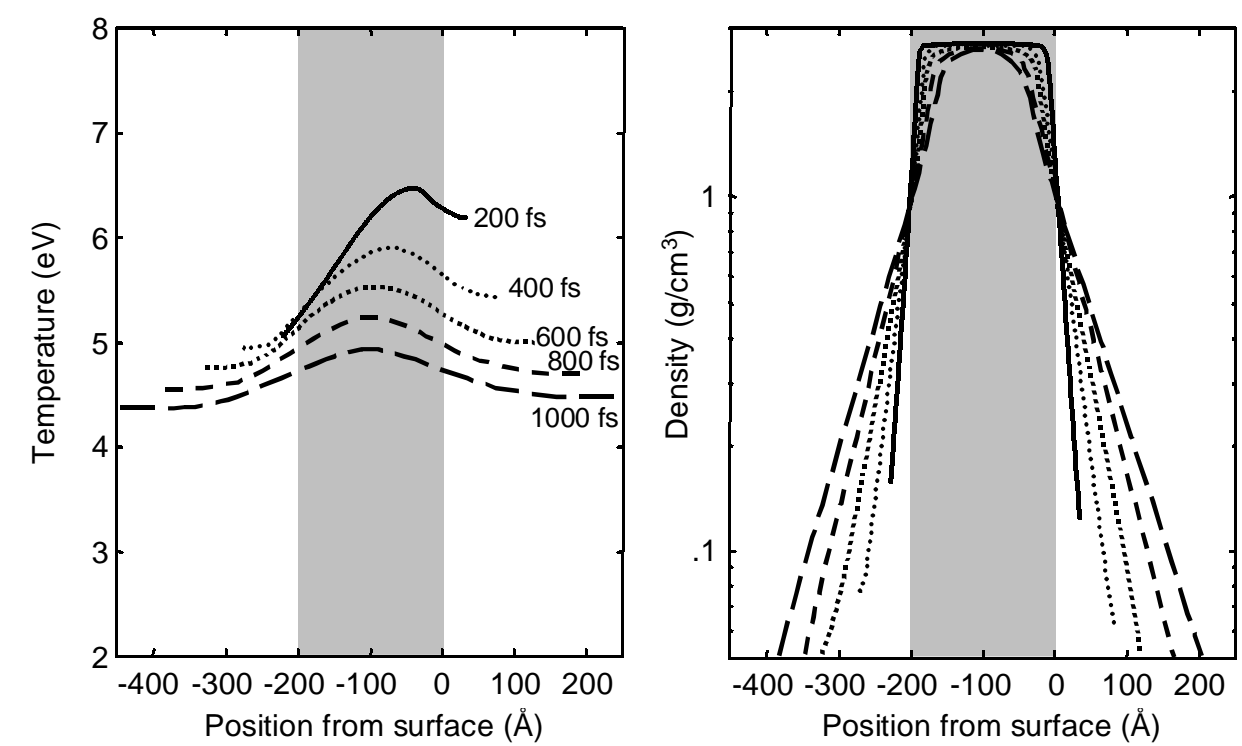

Figure 9. The predicted temperature profile (right) and the density profile (left) of a $200 \AA \mathrm{Al}$ foil at various times after heating by a $100 \mathrm{fs}, 0.3 \mathrm{~mJ}$ laser pulse. Note that changes occur by $\sim 200 \mathrm{fs}$ and that after 1 ps the temperature gradients are small.

Given the steps that have been taken at the ALS on beamlines 7.3.3 and now 5.3.1 it is clear that the capability of the UXS facility will provide a major step forward. The current warm dense matter experiments with the bend-magnet source are already pushing the beamline capabilities. The additional femtosecond $x$-ray flux of the UXS facility is necessary in order to: (1) improve the time resolution to $<1 \mathrm{ps,} \mathrm{which} \mathrm{is} \mathrm{important} \mathrm{for} \mathrm{defining} \mathrm{a} \mathrm{uniform} \rho$ and $T$, and

(2) to simultaneously enhance the signal to noise which is necessary for EXAFS experiments. The plan is to first verify the sub-nanosecond x-ray absorption dynamics of the Al foil using short-pulse laser heating. This will provide critical information about the behavior of the system under short-pulse excitation conditions. Beyond this, the high-flux short-pulse x-rays (from the UXS beamline) will be used to accurately probe the XANES and EXAFS spectra about the $\mathrm{K}$-edge of laser-heated $\mathrm{Al}$ at the appropriate time following excitation. Initial experiments and calculations will focus on $\mathrm{Al}$ because of the wealth of existing data and simulations for this element. Finally, the theoretical development effort at LLNL will be an important resource for a broad range of experimental activities at the UXS facility by providing advanced modeling capabilities for interpreting $\mathrm{x}$-ray spectral measurements, particularly in the complicated nearedge $\mathrm{x}$-ray absorption region.

\section{IV.4. Solid-Solid Structural Changes and Phase Transitions}

Transitions from one distinct solid phase or crystallographic structure to another represent another class of phase transitions in which the underlying dynamics are determined by atomic motion on the time scale of a vibrational period. In extended solids, transformations from one structure to another typically nucleate at defect sites. The macroscopic evolution of such transformations is then determined by the growth rate of the transformed region (at acoustic velocities), domain fracture, and the creation of multiple new defects and nucleation sites at the interface region. The complex evolution of such transformations make it difficult to understand 
the fundamental structural dynamics at the atomic scale occurring at each defect or nucleation site. By initiating such transitions homogeneously throughout a sample region (via direct optical excitation or via optically induced temperature or pressure jumps, for example), and probing the structural evolution with femtosecond x-ray diffraction or EXAFS, we can directly observe such ultrafast structural dynamics for the first time. This will lead to a better understanding of the atomic rearrangement and changes in bonding which are at the heart of such transitions.

Semiconductor nanocrystals are a unique model system for investigating solid/solid phase transitions since they are essentially free of defects. X-ray diffraction and EXAFS studies have shown that nanocrystals undergo a wurtzite (coordination=4) to rock salt (coordination=6) structural transformation at high pressure ( 6 GPa, see Fig. 10) [53-56]. The pressure-induced phase transition is entirely reversible, with essentially no loss of crystalline structure. This is convincing evidence that only single nucleation events occur in each crystallite, in contrast to the extended solid where multiple nucleation and domain formation complicate the dynamics. Thus, for sufficiently small particles, the phase transformation is reduced to the fundamental structural dynamics, which can then be directly probed using time-resolved x-ray techniques.

The scientific challenge is to understand not only the time scale, but the structural kinetics of such phase transformations. For example, the reversibility of the phase transformation suggests that it occurs homogeneously throughout each nanocrystal, analogous to the Néel coherent rotation model for magnetization reversal in a single magnetic domain. As shown in Fig. 10, the pressure dependence of the nanocrystal phase exhibits a pronounced hysteresis. In contrast with bulk phase transitions (in which the hysteresis width is determined by the types of defects present), in nanocrystals, the hysteresis is determined by the total number of unit cells which participate in the nucleation event. For instance, if the transition nucleates by one plane of the nanocrystal sliding against another, then the barrier to the transition is expected to scale with the area of the plane. The implication is that for sufficiently small nanocrystals, the transformation begins to behave as a molecular isomerization [54]. Furthermore, in this size regime the transformation may involve coordinated movement of the atoms throughout an individual nanoparticle (i.e. coherent motion on the time scale of a single vibrational period). Time-resolved EXAFS and/or x-ray diffraction offers unique capabilities for addressing these questions by directly probing the changes in atomic coordination and bond distances through the course of the phase transition. 

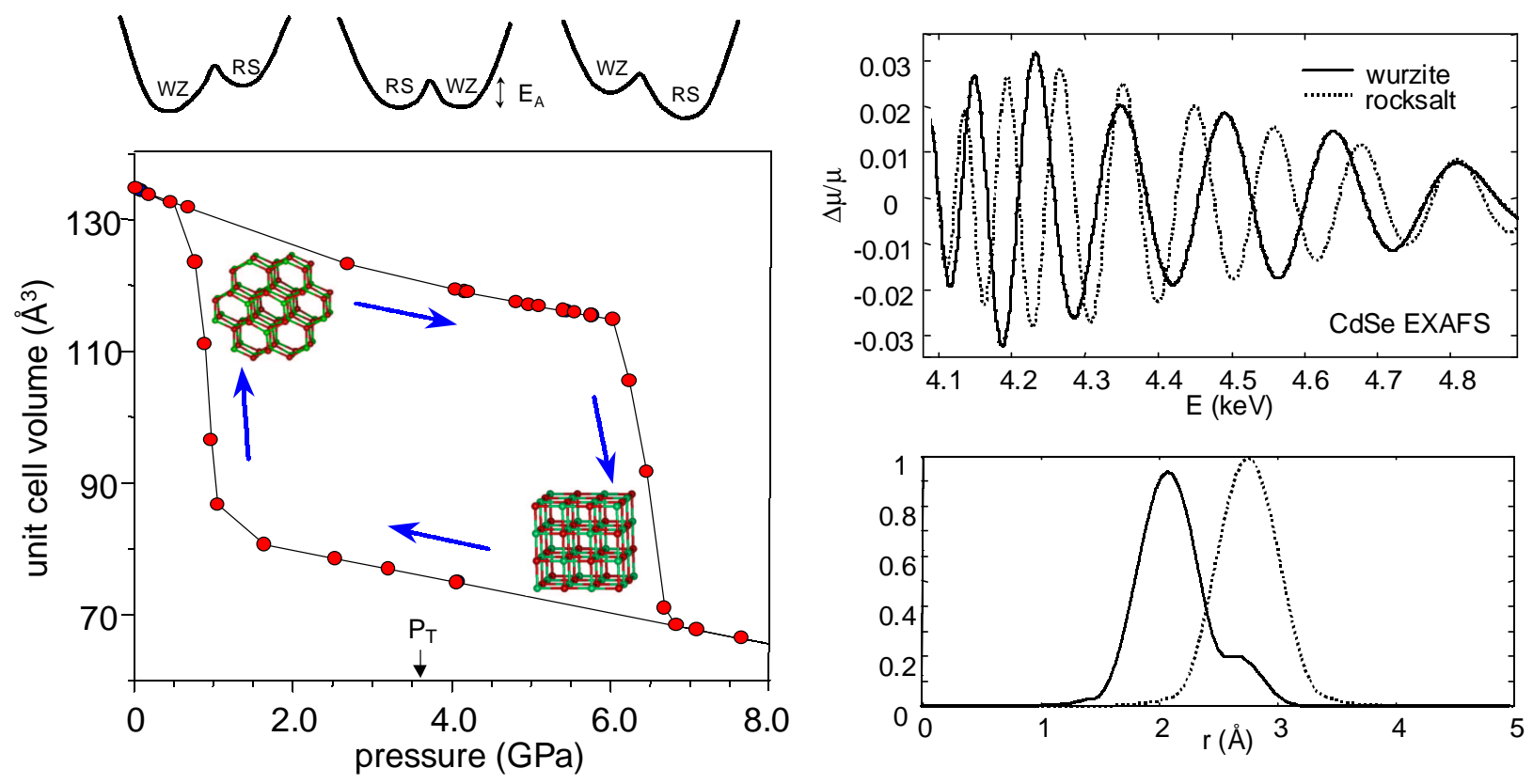

Figure 10. (left) Measurements of the wurtzite/rocksalt phase transformation in CdSe semiconductor nanocrystals. For CdSe particles $\sim 43 \AA$ in diameter, the thermodynamic transition pressure is $\mathrm{P}_{\mathrm{T}} \sim 4 \mathrm{GPa}$, and the activation energy is $\mathrm{E}_{\mathrm{A}} \cong 2 \mathrm{eV}$ (at $\mathrm{P}_{\mathrm{T}}$ ) [53,54]. (right) Model EXAFS calculations (top) and fourier transforms (bottom) for CdSe wurtzite and rocksalt structure $\left(\mathrm{Cd}_{\mathrm{I}^{-}}\right.$ edge).

A critical component for such experiments is the ability to trigger the phase transition simultaneously in a sufficient number of nanocrystals. Several promising approaches for optically triggering the phase transition are currently being explored. These approaches take advantage of what is already known about the kinetics and thermodynamics of the phase transition. As shown schematically in Fig. 10, the activation energy, $\mathrm{E}_{\mathrm{A}}$, can be manipulated via pressure. Furthermore, at a fixed pressure, the energetically favorable transition can be induced by a change in temperature [54] (i.e. the width of the hysteresis curve is temperature dependent). This allows one to choose an optimum condition (with a minimum potential barrier) for triggering the phase transition with an optical pulse. For example, operating at pressures below $\mathrm{P}_{\mathrm{T}}$ may enable one to transiently induce a wurtzite $\rightarrow$ rocksalt transition. Under such conditions, the relaxation time of the rocksalt $\rightarrow$ wurtzite back-transition is known to exhibit an Arrhenius dependence on the activation energy (as determined by pressure) [54]. The activation energy at $\mathrm{P}_{\mathrm{T}}$ has been measured to be only $1.5 \mathrm{eV}$ for $30 \AA$ diameter nanocrystals [54].

In a simple scheme, the phase transition may be triggered through femtosecond pulse laser heating of the sample. More sophisticated techniques include triggering the transition through optical excitation of coherent LO phonon modes in the excited state (via resonant absorption), or in the ground state (via impulsive Raman). Previous studies have shown that femtosecond optical pulses can excite strong coherent LO phonon modes due to enhancement of the Fröhlich coupling by quantum confinement effects [57]. Since the wurtzite/rocksalt transformation involves relative motion between basis atoms, it is likely that the LO phonon mode has a significant projection along the reaction coordinate of the phase transition. Finally, the modest pressure requirements $(<10 \mathrm{GPa})$ will enable the transition dynamics to be probed via 
X-ray diffraction or EXAFS (at the $\mathrm{Cd}_{\mathrm{I}}$-edge at $4 \mathrm{keV}$ ) in a diamond anvil cell, probing either through a thin diamond window or though a small diameter Be gasket $\left(1 / \alpha_{\mathrm{Be}} \cong 650 \mu \mathrm{m}\right.$ at $4 \mathrm{keV}$ ) [58]. Figure 10 (right) illustrates the distinct signature of this phase transition in the CdSe EXAFS spectra.

\section{Ultrafast X-ray Spectroscopy of Complex Materials}

Time-resolved x-ray measurement techniques offer powerful new tools for unraveling the complicated dynamical properties of "complex" materials. Complex materials include strongly correlated materials and man-made novel structures in which the effects of strong interactions and/or reduced dimensionality play an important role in determining the material properties. Ultrafast optical measurements of complex materials provide only limited information about the underlying dynamics because the optical properties in these systems are complicated functions of numerous degrees of freedom (charge, spin, lattice, etc.) which strongly interact. Complex materials are often multi-element and require element-specific or depth-sensitive probes for a thorough understanding. Furthermore, the energy scales of the elementary excitations cover a wide range, of which only a small portion can be covered by optical methods.

Strongly correlated materials exhibit many fascinating phenomena, such as colossal magnetoresistance $(\mathrm{CMR})$ or high- $\mathrm{T}_{\mathrm{c}}$ superconductivity, due to the strong interactions among charge, spin and lattice. A better understanding of the interaction between charge, spin, and lattice can be obtained by time-resolving their individual responses to external perturbations. For example, ultrafast lasers can instantaneously introduce carriers into the charge-ordered, antiferromagnetic, insulating ground state of heavily hole-doped CMR manganites. Measurements of the subsequent excitation and relaxation of the charge, spin, and lattice will provide new information about the physics of the CMR materials. The strength of the chargespin and charge-lattice interactions can be estimated from the time-scales of the excitation and relaxation, for instance. Ultimately, the form of the interaction Hamiltonians responsible for the dynamics can be inferred from these measurements.

Time-resolved measurement techniques can be implemented in virtually all x-ray spectroscopic methods, which have proven to be excellent probes of the static properties of complex materials. Time-resolved x-ray Bragg diffraction can be used to monitor changes in the long-range lattice order induced by carrier excitation via the Jahn-Teller (JT) interaction. Resonant time-resolved x-ray diffraction at the $\mathrm{Mn} \mathrm{K}$-edge $[59,60]$ will provide additional information on the dynamics of the $d$-orbitals of the manganese ions: the ordering of $\mathrm{Mn} d$ orbitals is believed to be an important ingredient in the formation of the charge stripes [61]. Time-resolved EXAFS can probe the evolution of the short-range lattice order, bond lengths, and bond angles. The dynamics of charge-ordering can be observed by time-resolved XANES [62] or by changes in the superlattice Bragg diffraction due to the charge ordering.

The utility of time-resolved x-ray measurement techniques for strongly correlated materials has been demonstrated by recent observation of the polaron dynamics in chargeordered $\mathrm{Nd}_{1 / 2} \mathrm{Sr}_{1 / 2} \mathrm{MnO}_{3}$ using time-resolved x-ray Bragg diffraction, as shown in Fig. 11 . Transient modifications in the lattice structure induced by the JT interaction are monitored following optical excitation with $150-\mathrm{fs}$, 800-nm laser pulses. The laser wavelength overlapped 
with the broad $\mathrm{Mn}^{3+}$ to $\mathrm{Mn}^{4+}$ charge-transfer band [63]. The x-ray rocking curve for Pbnm orthorhombic (112) reflection, measured $50 \mathrm{ps}$ after the laser pulse, is shown in the inset of Fig. 11 with the unperturbed rocking curve. The observed dynamics of the diffracted intensity at different points on the rocking curve are illustrated in the left panel of Fig. 11. The increases in both the shorter and longer wavelength sides of the rocking curve signify the development of compressive and expansive strains in the crystal.
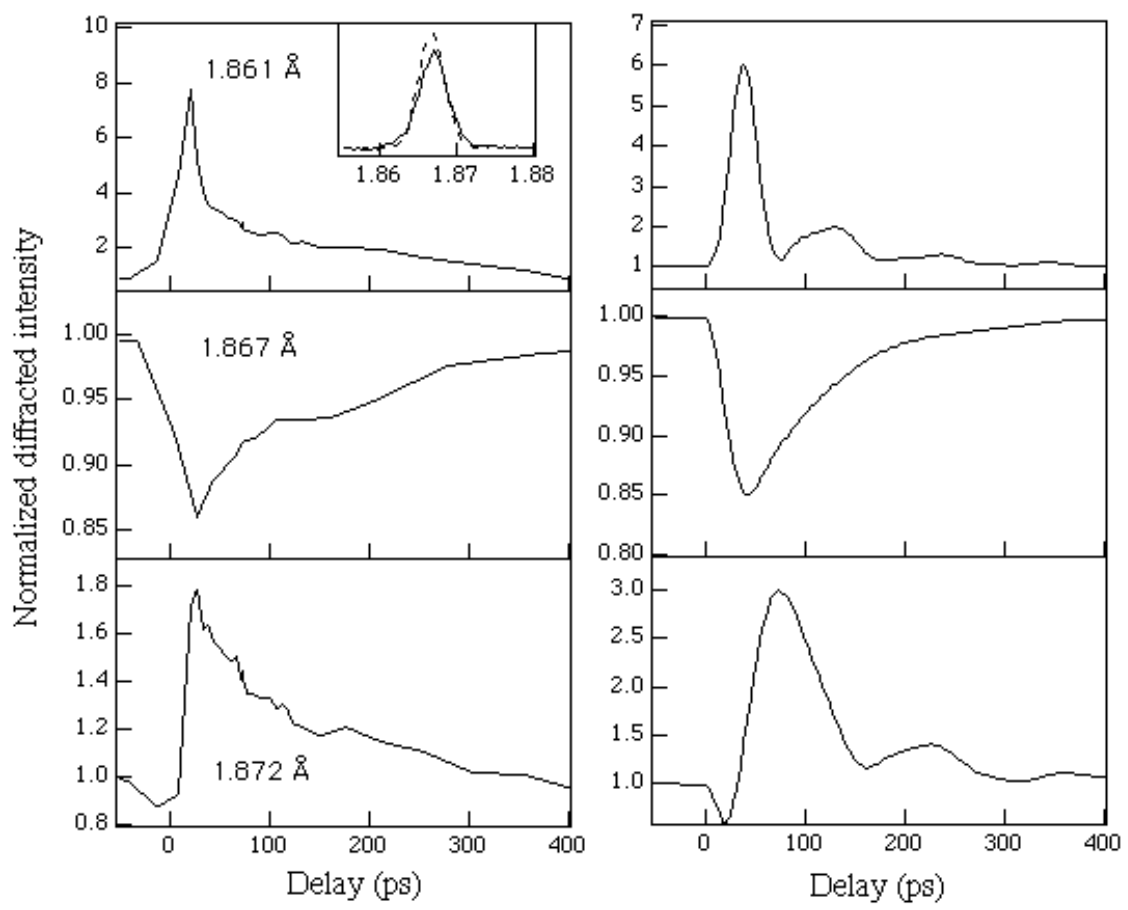

Figure 11. (left) The measured dynamics of the diffracted $x$-ray intensity at different points of the rocking curve. (right) Theoretical modeling of the data assuming laser-generated transient JahnTeller lattice distortion in the crystal. The inset in the left panel shows the rocking curve measured $50 \mathrm{ps}$ after the laser pulse (solid curve) and the unperturbed rocking curve (dashed curve). In the figure, the unperturbed x-ray intensity for each point of the rocking curve is set to unity. (Measured at ALS beamline 7.3.3).

The laser-induced modification of the $\mathrm{x}$-ray Bragg diffraction is a direct result of the strong coupling between the JT lattice distortion and the charge-transfer transition in this system. Impulsive (faster than the lattice can respond) charge-transfer from ions to ions creates the JT lattice distortions shown in Fig. 12. The laser-induced lattice modulation appears be anisotropic (unlike simple thermal expansion) as both the compressive and expansive components are observed in the data. The data is modeled under the assumption that the charge-transfer transition creates the JT distortions depicted in Fig. 12. Relative to the lattice plane, which corresponds to (101) of the cubic perovskite, these distortions amount to longitudinal expansion and transverse compression. The longitudinal and transverse strain associated with the JT distortions propagate into the crystal with the relevant speed of sound. Modifications of the diffracted intensity due to the strain waves are calculated and shown in the right panel of Fig. 11. From the calculation, the relaxation time of JT distortions is estimated to be $\sim 50 \mathrm{ps}$ and the JT interaction energy is $\sim 1 \mathrm{eV}$, independent of temperature. The fact that similar dynamics are 
observed between 20 and $300 \mathrm{~K}$ suggests that the JT interaction strength is larger than the bandwidth of the band derived from the itinerant $\mathrm{e}_{\mathrm{g}}$ electrons.

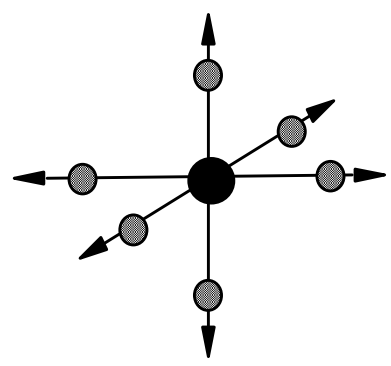

$\mathrm{Q}_{1}$

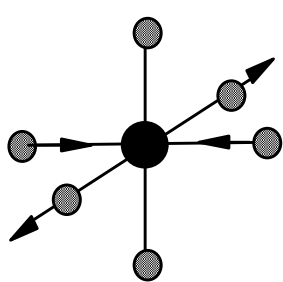

$\mathrm{Q}_{2}$

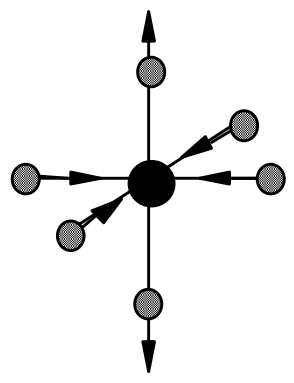

$\mathrm{Q}_{3}$

Figure 12. The Jahn-Teller distortion of the oxygen octahedra around Mn ions which strongly interact with the optical charge-transfer transition.

The proposed undulator beamline provides a much improved capability for studying the dynamics of strongly correlated materials compared to the current bend-magnet beamline. The additional $\mathrm{x}$-ray flux will allow time-resolved $\mathrm{x}$-ray powder diffraction measurements. This will significantly increase the scope of materials whose dynamics can be studied since novel complex materials are rarely available as high-quality single crystals. The bright sub-picosecond soft xray pulses are especially suited for studying the electronic and magnetic dynamics at the $L$-edges of these transition-metal compounds. The sensitivity of the streak camera measurements can also be greatly improved at soft $x$-ray range due to the enhanced quantum efficiency of the photocathodes.

\section{IV.5. Ultrafast Photochemical Reactions}

The conventional model of photochemical reactions in the condensed phase is based on the notion of a lowest-energy excited-state which is common to both reactant and photoproduct. In such a model, photoexcitation to the Franck-Condon region is followed by intramolecular vibrational relaxation (IVR) in the excited state followed by internal conversion (IC) or partitioning to photoproduct and reactant ground states. Such a model assumes that structural changes along the reaction coordinate are slow compared to IVR (which occurs on a picosecond time scale). A necessary consequence of such a model is that the reaction dynamics are determined by the lowest-energy excited state and are essentially independent of the initially excited Franck-Condon states.

The application of femtosecond lasers to photochemistry has demonstrated the inadequacy of such a model. Clear experimental observations of photochemical reactions involving structural changes on the time scale of a vibrational period include isomerization reactions in stilbene [64], bacteriorhodopsin [65], and rhodopsin [66-68], charge transfer reactions [69,70], photodissociation reactions in iodine [71,72], and myoglobin [73], to name just a few. Such, observations raise an important scientific question about the role of structural dynamics in influencing the reaction pathway. Recently ultrafast reactions have been described 
theoretically using dynamic, non Born-Oppenheimer models such as Landau-Zener tunneling [66,74], or conical intersections [75,76], which attempt to account for the role of structural (vibrational wavepacket) dynamics on the evolution of the transition state. Our present experimental understanding is limited by our inability to directly probe the molecular structure of these transition states during the course of a reaction. Femtosecond lasers have been successfully used to probe the optical properties of molecules in the transition state (as well as the ultrafast creation of photoproduct), because they can provide the necessary time-resolution. However, it is generally not possible to infer detailed structural information from optical properties. Optical properties provide only indirect information about the underlying atomic structure because visible light interacts with extended electronic states as opposed to core levels which are closely bound to the atomic nuclei.

\section{Organo-metallic Spin-Crossover Molecules}

The extension of EXAFS and XANES techniques to the femtosecond time domain opens the possibility of directly investigating the transition state of ultrafast chemical reactions. One particularly interesting class of molecules are the Fe-based organo-metallic complexes [77,78]. This is a model system for understanding the fundamental interplay between structural dynamics, charge-transfer, and spin-state interconversions. The spin-crossover transition which occurs in $\mathrm{Fe}^{\mathrm{II}}$ complexes is of particular interest because it is closely related to the electron transfer reactions in heme proteins. Of practical interest is the potential application of the light-induced excited spin-state trapping (LIESST) for opto-magnetic storage [79].

The Fe ${ }^{\text {II }}$ complex (illustrated in Fig. 13) consists of an Fe atom within a shell of organic ligands characterized by 6-fold coordination of $\mathrm{N}$ [78]. Optical excitation promotes an electron from the low-spin $\mathrm{Fe}^{\mathrm{II}}$ complex to a metal-to-ligand charge-transfer ( ${ }^{1} \mathrm{MLCT}$ ) state that is thought to be localized on a single ligand. The system subsequently undergoes a rapid spincrossover to a high-spin state $\left({ }^{5} \mathrm{~T}_{2}\right)$ of the $\mathrm{Fe}^{\mathrm{II}}$ complex. Femtosecond optical pump-probe experiments have demonstrated that the spin transition $(\Delta S=2)$ occurs in less than $700 \mathrm{fs}[77,80]$. The speed and efficiency of this reaction are inconsistent with the conventional model for such reactions (which assumes that the reaction proceeds from a thermally-equilibrated excited state) and suggests that the initial wavepacket dynamics in the Franck-Condon region play an important role in determining the reaction pathway. Static x-ray diffraction [78] and EXAFS measurements [81] demonstrate that the high-spin state is associated with significant $(\sim 10 \%)$ changes in the $\mathrm{Fe}-\mathrm{N}$ bond lengths. Model calculations suggest that the increase in $\mathrm{Fe}-\mathrm{N}$ bond lengths together with an increase in trigonal distortion facilitate the fast rate of spin interconversion in $[\mathrm{Fe}(\mathrm{tpen})]^{2+}$ by modifying the spin-orbit coupling [78]. 

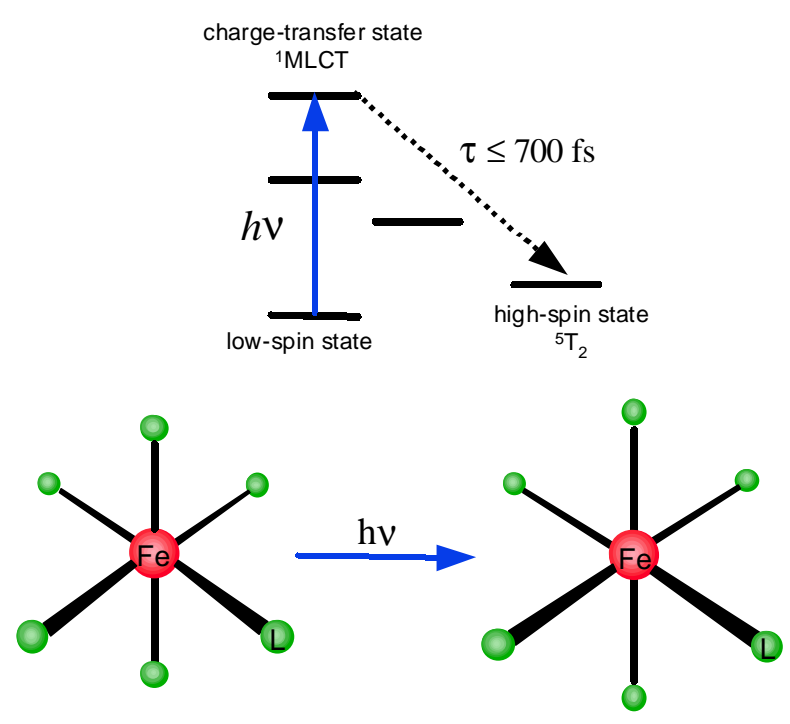
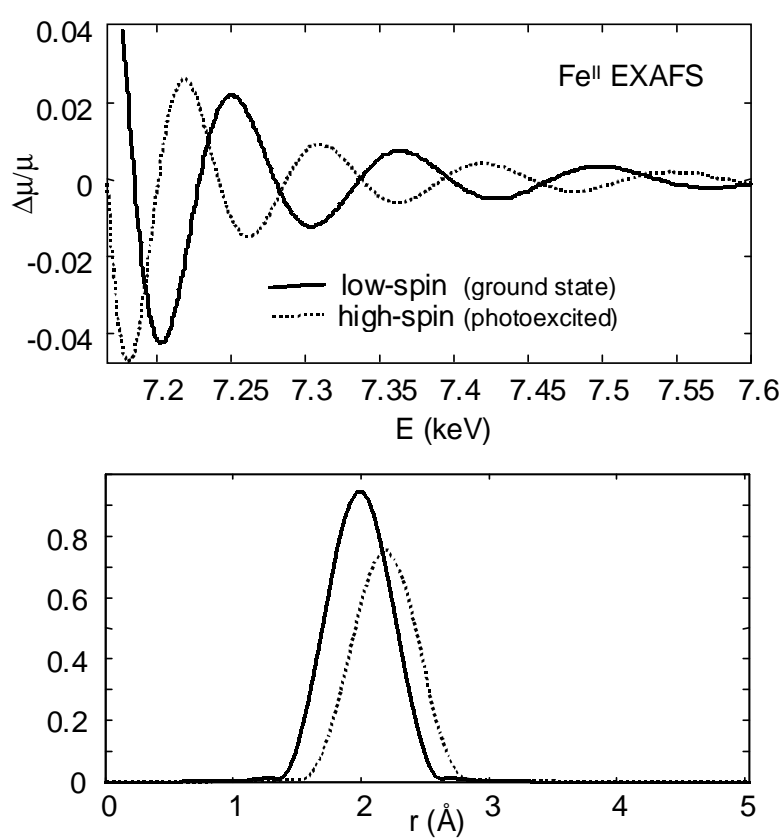

Figure 13. (left) Ultrafast spin-crossover reaction in $\mathrm{Fe}^{\mathrm{II}}$ organo-metallic complex. The high spin state is associated with an $\sim 10 \%$ increase in the Fe-N(ligand) bond distance. (right) Model EXAFS calculations (top) and corresponding Fourier transforms (bottom) for $\mathrm{Fe}^{\mathrm{II}}$ low-spin and high-spin structures.

A new understanding of the structural dynamics, and the role they play in the ultrafast spin-crossover conversion may be obtained by monitoring the changes in the $\mathrm{Fe}-\mathrm{N}$ bond distances during the reaction using time-resolved EXAFS measurements on the Fe K-edge at $7.1 \mathrm{keV}$ (or on the $\mathrm{L}_{\mathrm{I}}$-edge at $845 \mathrm{eV}$ ) in $[\mathrm{Fe}(\mathrm{tpen})]^{2+}$. Of significant advantage for these experiments is the wealth of existing static x-ray measurements of the temperature-isolated highspin and low-spin states [78,81-83], as well as femtosecond optical measurements of the excitedstate dynamics [77]. Initial experiments may be done in microcrystalline solids. A significant advantage of the superconducting undulator is the enhanced flux which will enable time-resolved EXAFS measurements in solution. In the case of the $\mathrm{Fe}^{\mathrm{II}}$ complexes, solution measurements are crucial to understanding the influence of solvent environment and intermolecular interactions on the ultrafast dynamics.

\section{Solvent-Solute Structural Dynamics}

Understanding chemical reactions in solution remains an important scientific challenge. In contrast to gas-phase reactions, solution reactions are strongly influenced by the solvent environment. Caging effects of the solvent can trap reactive species in close proximity allowing them to geminately recombine. In addition, coupling to the solvent can strongly influence the vibrational and electronic relaxation of the solute, and thereby influence the reaction pathway. The $\mathrm{I}_{2}$ photodissociation reaction is a simple model system for understanding molecular dynamics in solution, and there have been extensive theoretical and time-resolved experimental studies [71,84-88]. Measurements with femtosecond optical pulses indicate that vibrational coherence is maintained as photo-excited molecules in solution undergo curve-crossing to a dissociative state within a few hundred femtoseconds [71]. Geminate recombination has also 
been shown to occur within 2 ps [87]. Nevertheless, the dissociation dynamics, even for such an apparently simple diatomic molecule in solution, are not well understood. This is a consequence of the difficulty in inferring the structure of the molecular transition state from measurements of the optical properties. Time-resolved EXAFS measurements at the iodine $\mathrm{L}_{\mathrm{III}}$-edge $(4.6 \mathrm{keV})$ will provide the first direct measurement of the I-I bond distance during the dissociation process, and will provide new information about the subsequent solvent caging and geminate recombination.

The related tri-atomic system, $\mathrm{I}_{3}{ }^{-}$, exhibits more complicated structural dynamics during the photodissociation reaction:

$$
\mathrm{I}_{3}^{-}+h \mathrm{v} \rightarrow \mathrm{I}_{2}^{-} \text {(vibrationally excited) }+\mathrm{I}
$$

In solution (e.g., $\mathrm{H}_{2} \mathrm{O}$ ), geminate recombination occurs after vibrational relaxation from highlying nascent $\mathrm{I}_{2}^{-}$vibrational levels, and vibrational coherence has been measured to persist for several picoseconds [72,89-92]. Time-resolved XANES and EXAFS with femtosecond x-ray pulses will allow a direct observation of this rapid bond-breaking process involving all 3 iodine atoms. By resolving the structural evolution of the photodissociation, we can gain a better understanding of the steric interaction between the caged fragments $\mathrm{I}$ and $\mathrm{I}_{2}{ }^{-}$.

An important goal is to apply time-resolved x-ray techniques to understand the structural dynamics of more complicated reactions in a solvent environment. Of particular current interest is the photochemical reaction dynamics of aqueous chlorine dioxide $(\mathrm{O}-\mathrm{Cl}-\mathrm{O})$ which exists in stratospheric polar clouds and plays a significant role in sunlight-induced atmospheric chemistry [93] due to its ability to produce atomic $\mathrm{Cl}$. Recently, a number of groups have applied femtosecond optical techniques to understand the pathways and the dynamics of this reaction [93-96]. The data suggest that in solution, photo-dissociation and geminate recombination occur within 1 ps. However, there are several competing reaction pathways that appear to be solvent dependent [96].

$$
\begin{gathered}
\mathrm{OClO}+h v \rightarrow \mathrm{ClO}+\mathrm{O} \\
\mathrm{OClO}+h v \rightarrow \mathrm{Cl}+\mathrm{O}_{2} \\
\mathrm{OClO}+h v \rightarrow \mathrm{ClOO} \rightarrow \mathrm{Cl}+\mathrm{O}_{2}
\end{gathered}
$$

Optical measurements have not been able to clearly distinguish between these pathways due to the difficulty in uniquely identifying the optical absorption spectra of the various transient species. Solvent caging further complicates the problem since reactants that geminately recombine will have substantial excess vibrational energy in the electronic ground state. Thus, the corresponding optical absorption signature evolves on the picosecond time scale as the excess vibrational energy is coupled to the solvent. We can achieve a clearer understanding of OClO photodissociation by using time-resolved EXAFS and XANES to measure the time-scale of the reaction dynamics, and to distinguish between the various reactant species during the course of the reaction. EXAFS measurements at the $\mathrm{Cl} \mathrm{K}$-edge $(2.8 \mathrm{keV})$ can be used to monitor changes in the $\mathrm{Cl}$ coordination while XANES measurements can be used to distinguish between 
$\mathrm{OClO}, \mathrm{ClOO}$, and $\mathrm{Cl}$ due to their distinct absorption signatures and chemical shifts in the absorption edge.

The structural dynamics of the solute molecule is only one aspect of reactions in solution. Equally important is the structural response of the solvent environment since the mutual interaction between solvent and solute can influence the reaction dynamics. Recent attempts to address this issue using femtosecond optical pulses have focused on the solvated electron as a model probe of the solvent environment [97-102]. Analysis of the transient absorption and dephasing provide information about the statistical fluctuations of the solvent, but cannot provide any direct structural information. By using a solvated halogen anion as a probe, time-resolved EXAFS and XANES can be applied to directly capture the structural details of the solvent dynamics.

For example, the EXAFS structure of $\mathrm{Br}^{-}$or $\mathrm{I}^{-}$in water is quite pronounced due to the large coordination number of oxygen atoms surrounding the anion. An ultrashort optical pulse is then used to initiate the following reaction:

$$
\mathrm{X}^{-}+h \mathrm{v} \rightarrow \mathrm{X}_{\text {neutral }}+\mathrm{e}_{\text {solvated }} \quad(\mathrm{X}=\mathrm{I}, \mathrm{Br})
$$

thus photo-detaching the electron from the ion and generating a nascent radical and an electron (see Fig. 14). The local structural environment of the solvent will react to this ultrafast change in the solute charge distribution, and the caging $\mathrm{H}_{2} \mathrm{O}$ molecules will tend to repel from the halogen atom. While the response of the cage molecules occurs on an ultrafast time scale, the lifetimes of both the "wet" electron and the radical are mainly diffusion-limited (the probability for geminate recombination is very low). In an optical pump-probe experiment, the lifetime of the solvated electron following UV-photolysis of $\mathrm{I}^{-} / \mathrm{H}_{2} \mathrm{O}$ has been shown to include an 8 ps and a $60 \mathrm{ps}$ component [100]. The group of M. Chergui (U. Lausanne) has observed the same dynamics for $\mathrm{I}^{-}$, and for $\mathrm{Br}^{-}$. They have further verified that more than $\sim 25 \%$ of the photogenerated electrons survive on a nanosecond to microsecond time scale (depending on concentration). At low concentrations the neutral survival probability is expected to behave similarly, since the neutral loss reaction $\mathrm{I}+\mathrm{I}^{-} \rightarrow \mathrm{I}_{2}^{-}$[103] remains insignificant. At larger concentrations the loss reaction contributes to a reduced survival lifetime of I down to the picosecond regime, which can then be probed with femtosecond x-ray pulses. Therefore, this system offers several advantages for time-resolved x-ray measurements of solvent structural dynamics. First, the longer lifetime of the iodine radical together with the pronounced EXAFS signal enables us to measure the cage structure around a radical in solution for the first time. Second, the $200 \mathrm{fs}$ time resolution provided by the superconducting undulator beamline, will allow the ultrafast response of the solvent cage to the initial excitation of the charge-transfer to solvent (CTTS) state to be directly monitored via time-resolved EXAFS. 

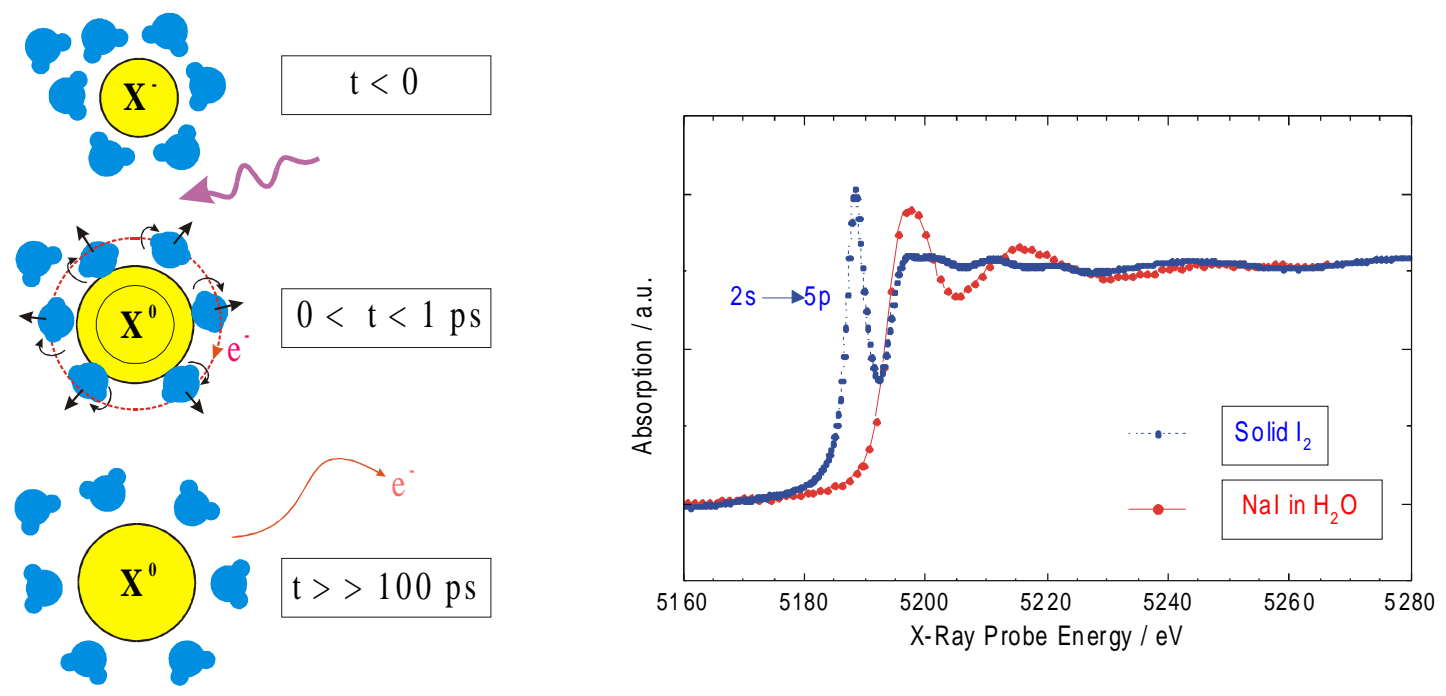

Figure 14. Schematic illustration of the halogen anion, $\mathrm{I}^{-}$, photo-detachment experiment in the liquid phase (left), and expected appearance of the $2 s \rightarrow 5 p$ resonance (right: dashed curve). This resonance has been observed in the X-ray absorption spectra of diatomic halogens at BM29 of the ESRF (A. Filipponi, unpublished data). The displayed halogen spectra will change shape after photolysis yielding the pre-edge resonance together with altered EXAFS modulations due to the changing I-O distance. The solid curve shows the measured EXAFS of $\mathrm{I}^{-}$in water under pumpprobe conditions (gated detection at $1 \mathrm{kHz}$ repetition rate - measured at ALS beamline 7.3.3).

Femtosecond optical experiments at the U. Lausanne have already verified that iodide can be efficiently ionized with UV pulses at $266 \mathrm{~nm}$ (via a one-photon process), or at $400 \mathrm{~nm}$ (via 2-photon absorption). A particular advantage of this model system is the enormous X-ray absorption changes expected after photolysis. Photodetachment of $\mathrm{I}^{-}$will free a $5 p$ hole. This hole is easily detectable in the XANES region due to its large absorption cross section. Figure 14 (right) illustrates this for the case of iodine. The anticipated $2 s \rightarrow 5 p$ transition is displayed by the Lorentzian-shaped pre-edge feature. The solid curve shows the static EXAFS spectrum of $\mathrm{I}^{-} / \mathrm{H}_{2} \mathrm{O}$, recorded by the Chergui group at BL7.3.3 of the ALS. The $5 p$ hole resonance is expected to be even stronger than the edge absorption itself.

\section{Structural Dynamics in Biological Systems}

Biological processes are ultimately governed by chemical reactions, and the fundamental time scale for the structural dynamics associated with these reactions (even in a protein environment) is a vibrational period. Thus, a thorough understanding of the basic driving mechanisms behind many biological processes requires direct structural measurements on an ultrafast time scale. Femtosecond optical techniques have been widely applied to investigate ultrafast biological processes [2], and coherent vibrational motion has been observed to play a role in biological reactions such as the first step in vision [66-68], photosynthetic charge transfer [69], and photodissociation in heme proteins [73]. Of course quantitative structural information requires x-ray measurements, and to date x-ray techniques have been applied on time scales from milliseconds to nanoseconds to investigate structural dynamics in biological systems such as heme protein [21,104], bacteriorhodopsin [22], and photoactive yellow protein [12]. Extending structural measurements of biological systems to the femtosecond time scale is a 
significant challenge due to demanding x-ray source requirements, but it is nevertheless an important long-term goal.

The UXS facility will be an important first step in this direction by enabling initial ultrafast time-resolved x-ray experiments in relatively simple biological systems such as metallo-proteins in solution. In addition to studying the metal atom in various oxidation states, it should be possible to apply EXAFS and XANES to determine the transient changes in the metal-ligand bond distance with high accuracy. For example, in heme proteins it is well established that a short optical pulse can induce photodissociation by absorption into the Soret bands. The first experiments will focus on the $\mathrm{Hb}-\mathrm{H}_{2} \mathrm{~S}$ system, which is currently investigated by optical pump-probe techniques at U. Lausanne (M. Chergui group) in collaboration with the University of Puerto Rico (UPR) at Mayagüez. This newly discovered sulfide-reactive hemoglobin from the clam (Lucina pectinata, $\mathrm{Hb} 1-\mathrm{H}_{2} \mathrm{~S}$ ) is a fascinating biological system which functions as a carrier protein for $\mathrm{H}_{2} \mathrm{~S}$ [105]. Read et al. first called attention to $\mathrm{Hb} 1-\mathrm{H}_{2} \mathrm{~S}$ in 1962 [106]. Nearly 30 years later it was finally possible to solve the static crystalline structure on a $\mathrm{Hb} 1$ compound with a resolution of $1.5 \AA$ [107]. The structural dynamics including the binding, transportation and release of the $\mathrm{H}_{2} \mathrm{~S}$ ligand, remain unknown. Here, time-resolved $\mathrm{x}$-ray absorption measurements may play an important role in furthering our understanding.

Probing structural dynamics in biological systems is a very challenging task, in part because of the low sample concentrations that are possible in real physiological media. Although dilute biological samples have already been successfully studied via EXAFS and XANES [108], the x-ray flux requirements are substantial. Estimates based on the performance of the proposed undulator beamline indicate reasonable data acquisition times for XANES and even EXAFS measurements at 30 ps resolution (see Appendix).

\section{IV.6. Atomic and Molecular Physics with Femtosecond Optical and X-ray Pulses}

Femtosecond optical techniques are widely used in gas-phase atomic, molecular, and optical (AMO) physics for time-resolved photoemission and ionization; generation and manipulation of non-stationary vibrational and Rydberg wavepackets; and in the investigation of atoms and molecules in high fields [2]. The availability of high-brightness femtosecond EUV and $\mathrm{x}$-ray pulses (in combination with femtosecond optical pulses) will open new areas of research in AMO physics by providing core-level photon energies (atom-specificity) and atomicscale resolution combined with ultrafast time resolution and high laser fields. These tools will enable for the first time, the direct characterization of molecular energy-level structure and bonding, as well as the photoionization dynamics as a function of the evolving molecular geometry.

\section{AMO Research with Femtosecond X-ray Pulses - Low Field Regime}

Femtosecond optical techniques are commonly used to study the time evolution of complex systems by preparing a state of interest with a pump pulse and interrogating the system as a function of the time delay with a probe pulse. In simpler systems for which the time evolution is known, pump-probe experiments can be used to study the behavior of the system as a function of geometry. For example, ultrashort optical pulses can prepare spatially localized 
vibrational wavepackets in the excited electronic state of a diatomic molecule. These nonstationary wavepackets then oscillate between the classical turning points of the potential energy surface (until coherence is lost via dephasing and vibrational energy relaxation). By probing such systems with a delayed ultrashort X-ray pulse, it is possible to study the electronic energy level structure as a function of the internuclear distance. This approach may also be applied to specific polyatomic molecules, thus allowing the study of the molecular electronic structure as a function of a normal coordinate of interest.

The ability to perform inner-shell x-ray studies as a function of molecular geometry will provide insight into a wide range of molecular phenomena. Near-edge absorption spectra often show a number of intense resonances which can be assigned to: transitions to unoccupied valence orbitals below threshold, to Rydberg orbitals, to states with multielectron excitation, and to shape resonances. It is of particular interest to understand how the positions, widths, and intensities of the resonances depend on the molecular geometry. The lowest energy resonances can often be correlated with the lowest unoccupied molecular orbital (LUMO) of the molecule, an important concept in models of chemical reactivity and the behavior of molecules on surfaces. The ability to probe these resonances as a function of geometry (for example, along a reaction coordinate) is expected to provide important new insight.

The internuclear distance $(\mathrm{R})$ dependence of the energy of the $\sigma^{*}$ shape resonances has been the subject of considerable discussion for more than 20 years. Studies of large classes of molecules have led some researchers to propose a quantitative correlation between bond length and $\sigma^{*}$ resonance position [62]. If valid, such a correlation would provide a powerful tool for the characterization of molecules on surfaces, in reactions, and in other novel environments. However, the existence of such a correlation has been questioned by a number of researchers [109]; the principal objection being the assignment (and even existence) of the $\sigma^{*}$ shape resonances in many of the molecules used to make the correlation. Some information on the $\mathrm{R}$ dependence of the $\sigma^{*}$ resonances can also be obtained from non-Franck-Condon distributions in vibrationally resolved photoelectron spectra. Unfortunately, such results are limited to very small molecules, require high-level theory to extract even semi-quantitative information, and are limited to a small range of R. Time-domain, pump-probe techniques will provide near-edge absorption spectra as a function of $\mathrm{R}$, and thus directly address all of these issues.

As an example, consider the near-edge absorption spectrum of $\mathrm{ICl}$ near the $\mathrm{I} \mathrm{L}_{\mathrm{I}}$ or $\mathrm{Cl} \mathrm{K}$ edges. Optical pump-probe experiments [110] on $\mathrm{ICl}$ have shown that it is possible to create wavepackets in the $\mathrm{A}^{3} \Pi_{1}$ state that oscillate between $\sim 2.3$ and $4.6 \AA$ with a vibrational period of $\sim 1$ ps. By probing such wavepackets with an ultrashort $\mathrm{x}$-ray pulse, it should be possible to record "fixed R" near-edge spectra over this large range of internuclear distances. It is important to note that the $\sigma^{*}$ resonances are predicted to depend strongly on $\mathrm{R}$--near $\mathrm{R}_{\mathrm{e}}$ in $\mathrm{N}_{2}$, a change in $\mathrm{R}$ of $0.3 \AA$ moves the resonance approximately $20 \mathrm{eV}$. Thus, in the proposed experiments, the $\sigma^{*}$ resonance should be shifted a considerable distance from the "probe-only" resonance position, improving the ability to distinguish the two. The extension of this approach to polyatomic molecules and their bending coordinates is expected to be quite rewarding. For example, although the $\pi^{*}$ resonances in triatomic molecules are thought to depend only weakly on bond length, theoretical considerations indicate that they may depend strongly on bond angle. 
There is little experimental information on this effect. Pump-probe experiments producing a wavepacket in the bending coordinate of such molecules would allow the recording of near-edge spectra as a function of bond angle, and directly address this issue. The $200 \mathrm{fs}$ duration of the ultrashort x-ray pulse is particularly well suited for such studies in heavier molecules (in which spatially localized wavepackets can be excited at relatively low vibrational frequencies). Pump probe studies of the bond angle dependence of the $\pi^{*}$ resonances in lighter molecules can still be done, but in this case the pump laser will excite a vibrational level (or group of levels) that is well-defined in the frequency domain.

One triatomic system which illustrates the idea of this approach is nitrogen dioxide $\left(\mathrm{NO}_{2}\right)$. Nitrogen dioxide is a nonlinear free radical with one electron outside a closed shell ion core. Although the ground electronic state of $\mathrm{NO}_{2}$ is bent, the ground state ion and the Rydberg states converging to it are linear. The first absorption band system of $\mathrm{NO}_{2}$ consists of a long progression of bands assigned to transitions to the $\mathrm{A}^{2} \mathrm{~B}_{1}$ state. This progression is extremely complex but can be simplified by cooling in supersonic molecular beams. By varying the pump laser wavelength, it should be possible to excite selected bands and subsequently to probe the inner-shell spectrum of the excited molecule with the femtosecond x-ray pulses.

Because the nuclei in a vibrating molecule spend most of their time at the classical turning points of their motion, the wave function in the time-independent picture has most of its amplitude near the classical turning points. Thus, in a nominally linear triatomic molecule with the bending vibration excited, the molecule will spend most of its time in the bent geometry with the bond angle determined by the bending quantum number. For the $v_{2}$ bending vibration in $\mathrm{NO}_{2}$, this means that the effective O-N-O angle will depend on the particular value of $v_{2}$, with larger $\mathrm{v}_{2}$, corresponding to smaller $\mathrm{O}-\mathrm{N}-\mathrm{O}$ angles. By performing inner-shell absorption studies on a series of vibronic levels with different values of $v_{2}$, we will be able to gain insight into the bond-angle dependence of the resonances in the near-edge region. Thus, detailed studies of the $\pi^{*}$ resonances will become possible. Similarly, because the two turning points of the asymmetric stretching vibration have the same geometry, excitation to a series of vibronic bands of the asymmetric stretch of $\mathrm{NO}_{2}$ would provide information on how these features depend on the relative bond lengths of the two N-O bonds. These experiments should be feasible based on the current ability to perform experiments on stable molecules and assuming that $10 \%$ of the sample can be excited to the level of interest.

Pump-probe techniques can also be applied to study the $\mathrm{R}$ dependence of the inner-shell orbitals. For example, photoelectron spectroscopy and photoelectron angular distributions could be used to probe how the molecular orbitals derived from the M-shell atomic orbitals in $\mathrm{I}_{2}$ change as the bond length varies from 2.5 to $5 \AA$, and in particular, to determine the degree to which the orbitals are localized on the individual atoms. Interestingly, at $2.5 \mathrm{keV}$ the $\mathrm{x}$-ray wavelength is $\sim 5 \AA$, the same size as the molecule at the outer turning point. In this situation, the dipole approximation is expected to be severely compromised. On the other hand, at the inner turning point the molecule is considerably smaller than the x-ray wavelength and the dipole approximation should be more accurate. Thus, at the inner turning point the wavelength of light is too long to distinguish the individual atoms, while at the outer turning point this is becoming possible. Under these conditions, the photoionization dynamics will be quite sensitive to the degree of delocalization of the atomic M-shell orbitals. 
In contrast to vibrational wavepackets, in which the nuclei of the molecule are spatially localized, it is also possible to prepare Rydberg states in which the Rydberg electron is spatially localized. Pump-probe experiments using such electronic wavepackets can then be used to study properties of the molecule as a function of the electron-ion core distance. For such experiments, it is desirable to stretch the excitation laser pulse to $\sim 1$ ps (with a bandwidth of about $30 \mathrm{~cm}^{-1}$ ). This requirement stems from the fact that on any classical orbit, the electron spends only a few picoseconds in the vicinity of the atomic nucleus. Classically, the Rydberg period scales as $n^{3}$, and for $\mathrm{n} 47$ the period is $\sim 15$ ps. By exciting a coherent superposition of several Rydberg levels around $n=47$, it is then possible to create a Rydberg wavepacket with a classical period of this magnitude. Most of this period is spent with the electron far from the ion core. Timeresolved x-ray absorption spectra measured during the outer excursion of the Rydberg wavepacket should reveal an inner-shell ionization spectrum similar to that of the corresponding bare ion. Conversely, x-ray absorption spectra obtained at time delays corresponding to the electron "in" the ion core should correspond more closely to that of the neutral species.

Time-resolved studies of Rydberg wavepackets have been performed previously with optical probes; however, the emphasis of these earlier studies has been primarily on the dynamics of the Rydberg electron (see, for example [111]). In the proposed experiments, the emphasis will be on the effect of the Rydberg electron on the inner-shell absorption spectrum of the core, particularly when the Rydberg wavepacket is localized in the core. As an example, in an alkali atom, single-photon excitation will prepare a wavepacket composed primarily of $n p$ Rydberg states, while two-photon excitation will prepare a wavepacket composed primarily of $n s$ and $n d$ Rydberg states. Because the short-range behavior of the $n p$ and $n s / n d$ wavefunctions are very different, they are expected to have different effects on the core electrons. The comparison of near-edge absorption spectra recorded when the Rydberg wavepackets are localized in the core should provide new insight into the correlated behavior of the core electrons.

Another example where ultrafast time-resolved core-level studies would be a powerful new tool in the chemical physics toolbox is in the study of the photodissociation of polyatomic molecules. Much attention has been given to studies of photofragmentation mechanisms [112]. Large molecules can have complex valence spectral features that are often impossible to uniquely assign to individual molecular orbitals. Core level spectra of large polyatomic molecules are typically much simpler and it is easy to separate features from different atoms due to the large energy shifts between core-level binding energies of different types of atoms. We propose to exploit this atomic site specificity in core-level studies of $\mathrm{Fe}(\mathrm{CO})_{5}$ by initiating the photoexcitation process with the femtosecond x-ray photon and by probing the time evolution of the molecular fragmentation using laser multiphoton ionization. The initial excitation can be done at the oxygen K-edge or at the $\mathrm{Fe} \mathrm{L}$ and K-edges. The ionic states can be well characterized by methods such as high resolution photoelectron spectroscopy. Using the x-ray photon as a pump will allow the deposition of energy at a specific location in the molecule, initiating electronic and nuclear rearrangements that can be probed with a variably delayed uvvisible pulse. Alternatively, the molecular photodissociation can be initiated with a femtosecond laser pulse and the time evolution of the molecular rearrangement probed with atomic site specificity using photoelectron spectroscopy with the ultrashort $\mathrm{X}$-ray pulse. 
In most of our studies we will use photoelectron spectroscopy since it is a universal detection technique that is a sensitive probe of the electronic structure of the molecule $[113,114]$. This method has already been applied in a time-resolved fashion for the study of ultrafast nonadiabatic processes [115-117] and spin-orbit coupling [113] in isolated polyatomic molecules.

Finally, the past decade has seen tremendous improvement in the peak power of lasers. We would also like to explore new frontiers in photochemistry, by investigating the effect of high field photons introduced to the interaction region on the above proposed experiments. Processes such as bond-selective chemistry can be explored in which a strong programmed optical field creates a rovibrational or electronic wavepacket in a molecule, which may induce a specific change in molecular chemistry. Multiple interfering pathways toward specific ionization or dissociation products have already been demonstrated [118].

\section{AMO Research with Femtosecond X-ray Pulses - High Field Regime}

Advances in high-power, femtosecond laser technology have enabled experimenters to subject matter to electromagnetic fields on the order of an atomic unit of field strength $\left(5 \times 10^{11} \mathrm{~V} / \mathrm{m}\right)$. Interesting new phenomena arise (high harmonic generation [119], laser particleacceleration [120], production of x-ray lasers [121], etc.) and physical systems can be placed in novel regimes of phase-space which create new problems and opportunities for the theorist. While 'high-intensity' matter-field interactions have been investigated for well over a decade, even the basic atom-field interaction is poorly understood: typically perturbation theory is inadequate since the field cannot be regarded as a small perturbation. Accordingly, one is forced to develop and/or test non-perturbative methods of describing complex systems.

The UXS facility provides a unique opportunity to study two important aspects of highintensity atom-field interactions. First, femtosecond x-rays can be used to probe the structure of an atom or molecule dressed by an intense laser field. While measurement of electron and ion spectra in 'laser only' experiments has shed light on the interaction of intense lasers with atoms [122-124], the energy-level structure of a tightly coupled atom-field system has not been directly probed and is not well understood in the high-field, non-perturbative regime. Using wavelength-tunable, femtosecond x-rays one can probe the structure of the laser-dressed valence states by driving transitions from well-defined and unperturbed core states to the laser-dressed valence states of interest. Since femtosecond laser pulses are used in high-field experiments (both to obtain high intensity and because atoms in intense fields exist only transiently before ionizing), femtosecond $\mathrm{x}$-ray pulses are needed to probe the dynamic system.

Second, x-ray absorption and electron/ion spectroscopies can be used to probe how intense laser fields modify both the interaction of $x$-rays with inner-shell electrons and the relaxation of an x-ray-excited or ionized atom. The filling of the inner-shell hole by Auger processes is very fast $(<2 \mathrm{fs})$. However the presence of the laser during relaxation will modify the autoionization of the upper states as well as the postcollision interaction, thereby changing the end products. In addition to its importance from a fundamental perspective, gaining knowledge on the relaxation of atoms and small molecules in intense femtosecond fields is an important stepping stone to understanding the processes by which more complex molecules that are x-ray excited come apart in intense fields. This situation is very likely to occur in experiments planned for fourth generation light sources. 


\section{Continuum Dressing}

A central aspect of understanding atoms and molecules dressed by intense laser fields is understanding the atom-field wavefunction. An exact analytic solution to the laser-field + Coulomb-field system remains an unsolved problem and one typically resorts to analytic or quasi-analytic solutions at various levels of approximation. Experimental confirmation of the model predictions is important. Continuum dressing experiments will be performed as an initial attempt to probe the atom-field wavefunction in the high-field regime.

Here an x-ray photon projects an electron from a core state into a laser-dressed continuum and one measures the resulting electron spectrum. Continuum scattering is attractive since it is the presence of the Coulomb potential which creates the theoretical difficulties and since the importance of the Coulomb potential can be adjusted by tuning the X-ray wavelength. Effects of the Coulomb potential are expected to be most significant near an x-ray photoabsorption edge (where the photoelectron energy is low and more easily perturbed by the Coulomb potential) [125]. One can therefore begin far from an absorption edge (where the Coulomb potential can be neglected and physics is relatively well understood) and subsequently tune near an absorption edge where effects of the Coulomb potential become important.

Preliminary investigations far from threshold (Coulomb potential unimportant) have been conducted in France [126] and at LBNL [127]. In the LBNL experiments femtosecond VUV pulses ( $\sim 30 \mathrm{eV}$, from high harmonic generation) photoionize a helium gas sample in the absence (Fig. 15, thin line) or presence (Fig. 15, thick line) of a 'dressing' laser pulse. The dressing laser pulses stimulate transitions within a laser-dressed continuum producing additional peaks in the photoelectron spectrum. In addition, the spectrum is observed to shift to lower energy due to an AC Stark shift of the continuum level. A comparison to theory shows that to within the accuracy of the data $(\sim 20 \%)$ the process is well described by Volkov wavefunctions.

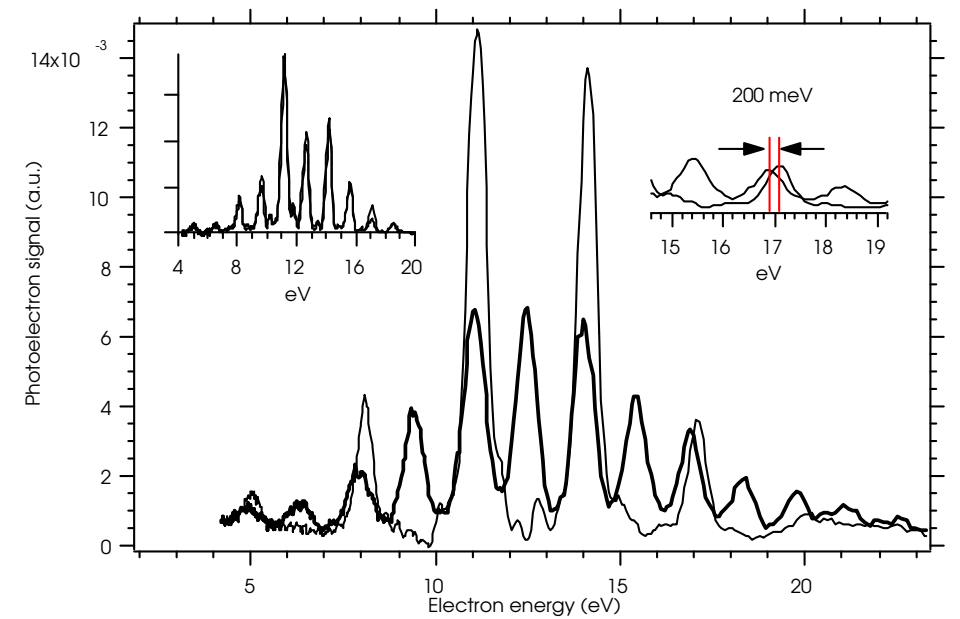

Figure 15. Helium VUV photoelectron spectra obtained in the absence (thin line) and presence (thick line) of a laser field. Right inset: laser-induced spectral shift. Left inset: comparison to theory [127].

The UXS facility offers a unique opportunity to extend this preliminary work to study effects of the Coulomb potential. One can therefore directly assess (via experiments) the need- 
for and accuracy-of more sophisticated approximations to the atom-field wavefunction (such as Coulomb-Volkov wavefunctions [125]). Continuum dressing will be studied by working at moderate laser intensities $\left(<10^{14} \mathrm{~W} / \mathrm{cm}^{2}\right)$ and with core-state electrons (i.e. states where it is a good approximation to neglect effects of the laser). Both angle-integrated (initially) and angleresolved (subsequent) x-ray photoelectron spectra can be measured as a function of the X-ray wavelength (and laser intensity). An inert gas sample such a neon is an attractive candidate for study since its $1 s$ core state is deeply bound $(\sim 870 \mathrm{eV})$ yet well within the range of available $\mathrm{x}$-ray photon energies. This core state should be relatively unperturbed for laser intensities less than $10^{13}-10^{14} \mathrm{~W} / \mathrm{cm}^{2}$. These continuum-scattering experiments will probe the continuum states of the atom-field system and will help to develop an understanding of the more complicated bound state structure of laser-dressed atoms and molecules.

\section{Bound state structure and modification of $x$-ray absorption}

Arguably one of the most interesting and challenging aspects of these AMO studies will be the study of the bound state structure of a femtosecond laser-dressed atom and the evolving distribution of population within this tightly bound light-matter system. While the strong interaction of light with matter has been investigated for decades, these studies have typically relied on resonances to enhance the atom-field coupling; here the major changes to the atomic structure are directly related to single-electron interactions with the field. In the currently proposed AMO studies we rely on high field intensity rather than resonances to access the strong-interaction regime.

One can expect modifications to the (field-free) bound state structure through mechanisms which include Stark shifts (and associated transient multiphoton resonances [122]), laser-dressing of bound states, and the possible mixing of states via multiphoton couplings (multiphoton couplings may even be sufficiently strong to permit observation of the multiphoton analog to Autler-Townes splitting [128]). Importantly, since an atom is a multielectron system that responds as a whole when perturbed by a laser field, modifications to the atomic structure by a femtosecond laser (acting on the outer electrons only) can indirectly extend to the inner-shells through electron correlation. These modifications are sizable and can modify dramatically the interaction of the atomic system with soft or hard x-rays. For example the binding energy of the $2 p$ electron varies from $38 \mathrm{eV}$ for a ground state $\mathrm{Na}$ atom to $44.9 \mathrm{eV}$ when the outer electron is promoted to $5 s$ using two dye lasers [129-131]. This represents an energy shift of $6.9 \mathrm{eV}$ for the $2 p$ core level, a shift that is much larger than the corresponding shifts due to the laser-induced Stark effect for the $2 p$ electron at the laser power of interest here $\left(\sim 10^{14} \mathrm{~W} / \mathrm{cm}^{2}\right)$. Our goal here is to extend the measurements of inner-shell photoionization and target relaxation to the time domain using femtosecond laser pulses in combination with femtosecond x-rays. An important difference with the $\mathrm{cw}$ laser experiments done to date is that the intense femtosecond laser fields will interact with many electrons of the atom and strongly perturb the structure and the population of the outer states. Although $\mathrm{x}$-ray induced inner vacancies relax on a much shorter time scale than the laser pulse width, the complete relaxation of the atom through autoionization will be modified by the presence of the strong laser field and can occur on a much longer time scales. Postcollision interaction and recapture of the photoelectron following the relaxation of an atom with a core hole has been extensively studied over the last decade. The postcollision interaction, autoionization and shake up processes involving the outer-shells have an important effect on the charge state distribution of the ions. The influence of an intense laser on these processes will be probed using x-ray absorption and electron/ion spectroscopies. Significant 
modifications due to the presence of a laser are expected and should, for instance, lead to very different charge state distributions and electron distributions for a field free atom versus laserdressed atom.

\section{Relation to U.S. Department of Energy Programs}

The Ultrafast X-ray Science facility supports the Strategic Plan of the Office of Science in several respects. The scientific motivation driving this facility is the need to understand the behavior of matter at the fundamental level - that is understanding structure at the atomic level within the fundamental (femtosecond) time scale in which structure evolves via the making and breaking of chemical bonds and the rearrangement of atoms. This research is important to condensed matter physics, chemistry, AMO physics, materials science, and biology. Structural dynamics ultimately dictate the genesis of unique states of matter, novel microstructure and nanostructures, as well as the pathways for chemical reactions, and even the function and efficiency of biological processes. One of the motivating factors behind the National Nanotechnology Initiative is the goal of controlling and manipulating matter at the atomic scale. Understanding the basic structural dynamics of matter is an important step toward that goal. Furthermore, the UXS facility supports specific existing BES programs within the Division of Materials Sciences, as well as the Division of Chemical Sciences. These include programs in ultrafast dynamics in condensed matter, nanostructure materials, chemical dynamics, and AMO physics. The UXS facility is also in support of current DOE Defense Programs research in highenergy-density physics.

Finally, the UXS facility is consistent with the DOE mission to provide national user facilities and research instrumentation that are beyond the capability and scale of what can be achieved in university laboratories. The UXS beamline at the ALS represents a significant facility enhancement for an existing national user facility by improving the measurement timeresolution that can be achieved at a $3^{\text {rd }}$ generation synchrotron by several orders of magnitude. This approach has been strongly endorsed by the BESAC Panel on Novel Coherent Light Sources (S. Leone - chair). The report of this panel specifically recognized that an important capability for future light sources is to combine ultrafast time resolution with structural information. Furthermore, one of the five key recommendations of the panel was "better utilization of $3^{\text {rd }}$ generation sources and table-top lasers as proving grounds for innovative experiments planned for light sources of the future" [28]. The UXS facility pushes the practical limits for ultrafast $\mathrm{x}$-ray measurements at existing $3^{\text {rd }}$ generation synchrotrons, and will open a new area of scientific research. The facility will play a critical role in developing a scientific field that will in the future take advantage of a $4^{\text {th }}$ generation source providing ultrashort $\mathrm{x}$-ray pulses. In the long term, the unique capability of the UXS facility for time-resolved measurements at high repetition rates (advantageous for signal averaging) will complement future X-ray FEL sources that are expected to operate at low repetition rates and high peak flux.

\section{Technical Description of the UXS Facility}


A detailed floor plan for the UXS facility at the ALS (beamline 6.0.1) is shown in Fig. 16. The superconducting undulator occupies straight-sector 6 of the storage ring. The hutch houses two experimental endstations (ES1 and ES2). Adjacent to the hutch is an enclosed area for the optical tables and the femtosecond laser system.

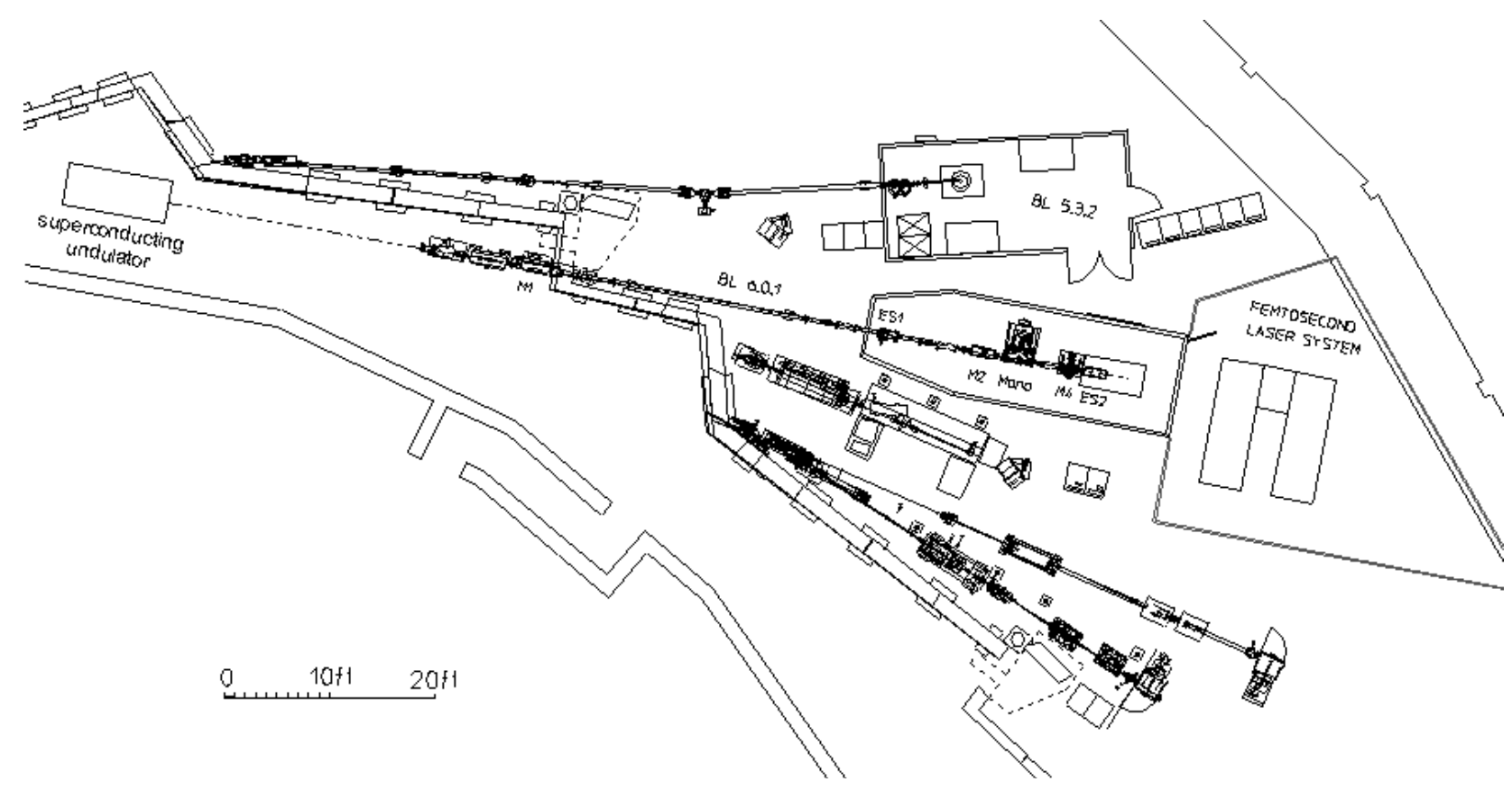

Figure 16. Layout of the Ultrafast X-ray Science facility (beamline 6.0.1) in sector 6 at the Advanced Light Source.

\section{VI.1. Generation of Femtosecond Synchrotron Pulses}

The duration of $\mathrm{x}$-ray pulses generated by synchrotrons is determined by the duration of each stored electron bunch, typically greater than $30 \mathrm{ps.} \mathrm{Storage} \mathrm{of} \mathrm{ultrashort} \mathrm{bunches} \mathrm{of}$ appreciable charge in a ring is problematic due to the effects of bunch-induced wakefields which give rise to bunch lengthening and other instabilities [132]. However, short electron bunches can exist in a storage ring for a limited time. We have recently demonstrated experimentally that a femtosecond laser pulse can be used to create femtosecond time structure on a long electron bunch via energy modulation. The energy modulation is then used to create an ultrashort slice of electrons that is spatially separated from the long bunch. Femtosecond x-rays are generated from the ultrashort electron slice [4,30]. Natural energy damping of the stored electrons allows the electron bunch to completely recover between laser interactions. Furthermore, because the modulation is created by interaction with a femtosecond optical pulse, there is absolute synchronization between the x-ray probe pulses and a convenient pump source for triggering dynamic processes.

Figure 17 shows a schematic illustration of the bunch modulation which is accomplished by co-propagating a femtosecond laser pulse with the stored electron bunch through a wiggler. The high electric field of the femtosecond laser pulse $\left(\sim 10^{9} \mathrm{~V} / \mathrm{m}\right)$ produces an energy modulation in the underlying electrons as they traverse the wiggler (electrons are accelerated or decelerated 
depending on the optical phase $\phi$ as seen by each electron at the entrance of the wiggler). The optimal interaction occurs when the central wavelength of the spontaneous emission from an electron passing through the wiggler, $\lambda_{S}=\lambda_{\mathrm{W}}\left(1+\mathrm{K}^{2} / 2\right) / 2 \gamma^{2}$, satisfies the resonance condition, $\lambda_{S}=\lambda_{\mathrm{L}}$, where $\lambda_{\mathrm{L}}$ is the laser wavelength, $\lambda_{\mathrm{W}}$ is the wiggler period, $\gamma$ is the Lorentz factor, and $\mathrm{K}$ is the deflection parameter of the wiggler [133]. In addition, the spectrum and transverse mode of the laser beam must match the spectrum and transverse mode of the spontaneous radiation from the electron passing through the wiggler. Under these conditions, the electron energy modulation, $\Delta \mathrm{E}$, is given by [30].

$$
\Delta E \cong 2\left(A_{L} A_{W} \frac{M_{W}}{\sqrt{2} M_{L}} \eta_{e m i t}\right)^{1 / 2} \cos \phi \quad \text { for } \quad M_{L} \geq \frac{M_{W}}{\sqrt{2}}
$$

where $A_{L}$ is the laser pulse energy, $M_{W}$ is the number of wiggler periods, and $M_{L}$ is the laser pulse length in optical cycles (FWHM). $A_{W} \cong 4.1 \alpha \hbar \omega_{L} K^{2} /\left(2+K^{2}\right)$ is the energy spontaneously radiated by a single electron passing through the wiggler [134], where $\alpha$ is the fine structure constant, and $\omega_{\mathrm{L}}=2 \pi \mathrm{c} / \lambda_{\mathrm{L}}$. The coefficient, $\eta_{\mathrm{emit}} \approx 0.7$, accounts for the non-zero electron beam size $[4,29]$. For the UXS beamline, simultaneous operation with protein crystallography beamline 5.0 requires that the wiggler operate at minimum gap (to maintain the wiggler flux at $1 \AA$ ). Under these conditions, the laser is in resonance with the third harmonic of the undulator, $\lambda_{\mathrm{S}}=3 \lambda_{\mathrm{L}}$, and Eq. 1 becomes:

$$
\Delta E \cong 2\left(\frac{A_{L}}{n^{4 / 3}} A_{W} \frac{n M_{W}}{\sqrt{2} M_{L}} \eta_{\text {emit }}\right)^{1 / 2} \cos \phi \quad \text { for } M_{L} \geq \frac{n \cdot M_{W}}{\sqrt{2}}
$$

where $n$ is the undulator harmonic. Based on Eq. 2, operating in resonance with the third wiggler harmonic $(n=3)$, we estimate that a $75 \mathrm{fs}$ laser pulse (FWHM of the intensity, or $\mathrm{M}_{\mathrm{L}}=40=2.12 \mathrm{M}_{\mathrm{W}}$ ) with a photon energy of $1.55 \mathrm{eV}$, and a pulse energy $\mathrm{A}_{\mathrm{L}} \approx 600 \mu \mathrm{J}$ will produce an energy modulation amplitude $\Delta \mathrm{E} \approx 9 \mathrm{MeV}$ using a wiggler with $\mathrm{M}_{\mathrm{W}}=19$ periods. The significance of the energy modulation $\Delta \mathrm{E}$, is in comparison with the rms beam energy spread, $\sigma_{\mathrm{E}}$ (for the ALS operating at a nominal energy of $1.9 \mathrm{GeV}, \sigma_{\mathrm{E}}=1.5 \mathrm{MeV}$ ). This is critical since it determines the efficiency with which the femtosecond synchrotron pulses can be isolated from the long synchrotron pulses, as described below. Note that since $\sigma_{\mathrm{E}} \propto \gamma^{2}$, Eq. 2 implies that for a fixed ratio of $\Delta \mathrm{E} / \sigma_{\mathrm{E}}$, the required laser pulse energy must scale as $\mathrm{A}_{\mathrm{L}} \propto \gamma^{4}$. Thus this technique is particularly well suited for low-energy $3^{\text {rd }}$ generation synchrotron storage rings. 


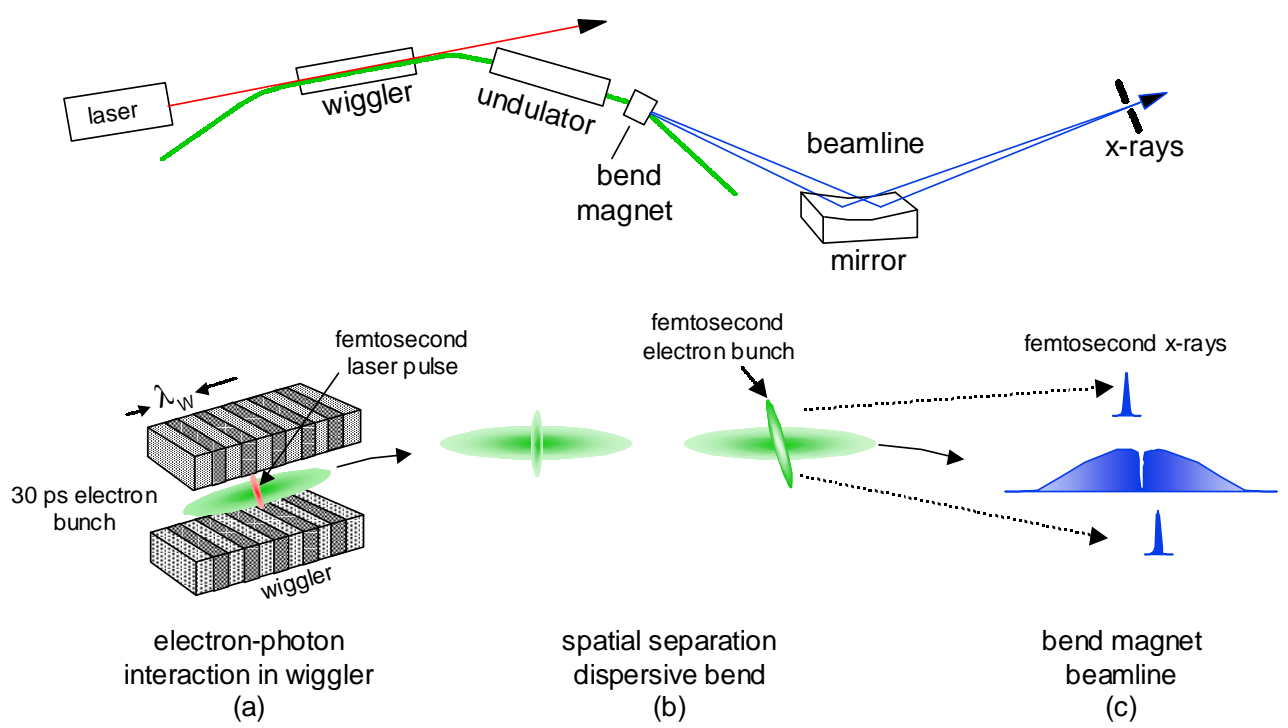

Figure 17. Schematic illustration of the technique for generating femtosecond synchrotron pulses, (a) laser/electron beam inter-action in resonantly-tuned wiggler, (b) separation of accelerated femtosecond electron slice in a dispersive section, (c) generation of femtosecond x-rays at an undulator or bend-magnet beamline.

As described above, the laser-induced energy modulation can be more than five times larger than the rms beam energy spread at the ALS, and only a thin slice of electrons (temporally overlapped with the laser pulse) experience this modulation. The energy-modulated electrons are spatially separated from the rest of the electron bunch, in a dispersive section of the storage ring, by a transverse distance that is several times larger than the horizontal size of the electron beam (Fig. 17b). For the UXS facility, a vertical dispersion bump will be incorporated in the storage-ring lattice upstream of the superconducting undulator (see section VI.6). Thus, energy modulated electrons will be vertically displaced, and the extremely low vertical emittance of the storage ring will thus allow for optimum separation of the femtosecond $\mathrm{x}$-ray pulses. Finally, by imaging the synchrotron radiation from the displaced electrons to the experimental area, the femtosecond X-rays can be separated from the long-pulse using an aperture (Fig. 17c). (The relatively narrow spectral bandwidth of the undulator emission provides an additional means of isolating the femtosecond x-ray pulses, i.e. by photon energy, see section VI.3). Furthermore, the extraction of an ultrashort slice of electrons leaves behind an ultrashort hole or "dark pulse" in the core of the electron bunch (Fig. 17c). This dark pulse will appear in the generated x-rays, and can also be used for time-resolved spectroscopy.

The average flux and brightness of the femtosecond $\mathrm{x}$-rays is determined from the full undulator flux and brightness scaled by three factors: $\eta_{1}=\tau_{L} / \tau_{e}, \eta_{2}=f_{L} / f_{B}$, and $\eta_{3} \approx 0.2$, where $\tau_{L}$ and $\tau_{e}$ are the laser pulse and electron bunch durations, $f_{L}$ and $f_{B}$ are the laser and electron-bunch repetition rates, and $\eta_{3}$ accounts for the fraction of electrons that are in the proper phase of the laser pulse to get the maximum energy exchange suitable for creating the large transverse separation. Synchrotron radiation damping provides for recovery of the electron beam between interactions. Since the laser interacts sequentially with each bunch, the time interval between interactions is given by $\mathrm{N}_{\mathrm{B}} / f_{\mathrm{L}}$ where $\mathrm{N}_{\mathrm{B}}$ is number of bunches in the storage ring. Furthermore, since the bunch slice is only a small fraction of the total bunch an interaction 
interval of $\sim 3 \mathrm{msec}$ ( $30 \%$ of the storage ring damping time for the ALS [135]) is sufficient to allow recovery of the electron beam between laser interactions [30]. Thus, with 300 bunches in the storage ring, femtosecond $\mathrm{x}$-rays can be generated at repetition rates as high as $100 \mathrm{kHz}$ without adversely affecting the other beamlines at the ALS. Based on the known parameters of the ALS at a beam energy of $1.9 \mathrm{GeV}$ [135] and the specifications for the proposed superconducting undulator $\left(1.5 \mathrm{~m}\right.$ long, $3 \mathrm{~cm}$ period, $\mathrm{B}_{\max }=1.5 \mathrm{~T}$ - see section VI.2) with $\eta_{1} \eta_{2} \eta_{3} \approx 5 \times 10^{-8}$, (e.g. laser pulses of $100 \mathrm{fs}, 600 \mu \mathrm{J}$ at $40 \mathrm{kHz}$ ) we expect an average femtosecond flux of $\sim 10^{8} \mathrm{ph} / \mathrm{sec} / 0.1 \% \mathrm{BW}$, and an average brightness of $\sim 10^{11} \mathrm{ph} / \mathrm{sec} / \mathrm{mrad}^{2} / \mathrm{mm}^{2} / 0.1 \% \mathrm{BW}$ over a spectral range from 0.2 to $10 \mathrm{keV}$ (see Figs. 18 and 19). Note for comparison that the Thomson-source at the ALS used for the time-resolved diffraction experiments described in section IV.2 provided an average femtosecond flux $\sim 2 \times 10^{3} \mathrm{ph} / \mathrm{sec} / 0.1 \%$ and an average brightness of $\sim 3 \times 10^{3} \mathrm{ph} / \mathrm{sec} / \mathrm{mrad}^{2} / \mathrm{mm}^{2} / 0.1 \% \mathrm{BW}$. Also shown in Figs. 18 and 19 is the flux and brightness available in 30 ps pulses for pump-probe experiments at $40 \mathrm{kHz}$ repetition rate.

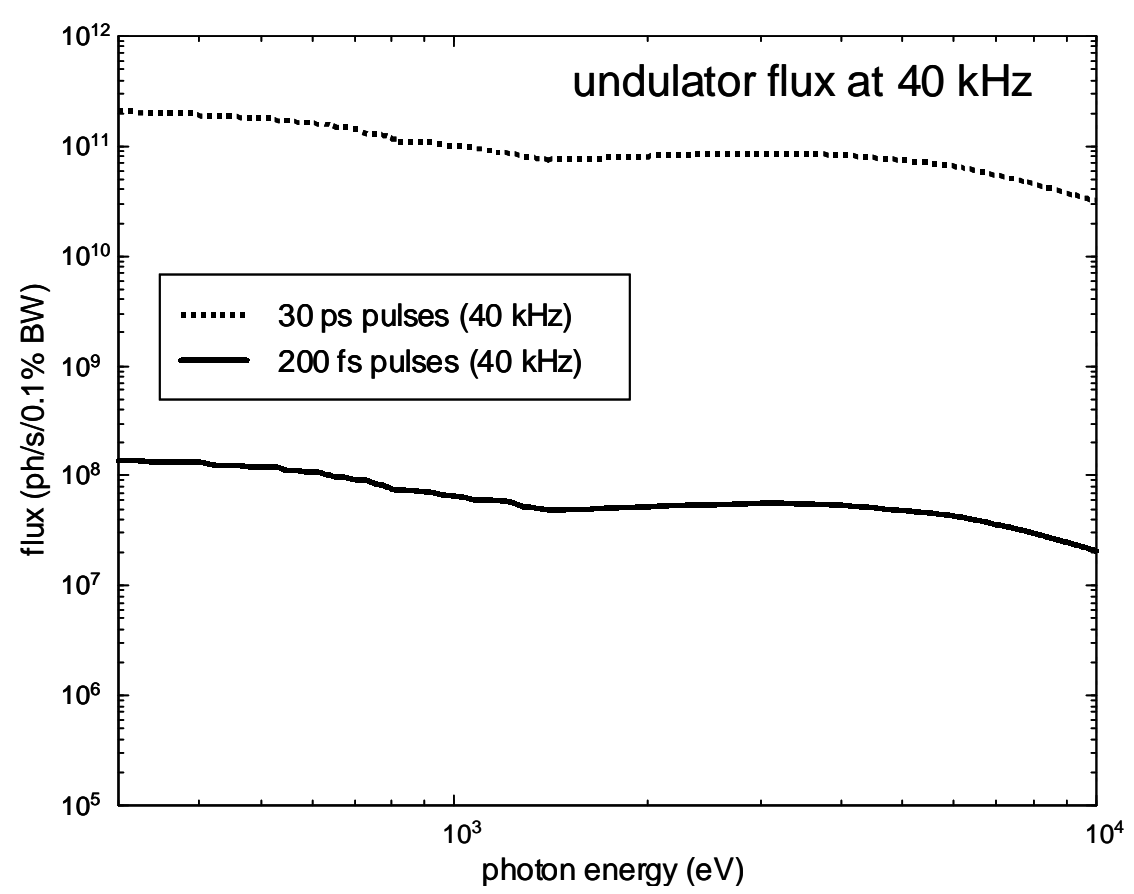

Figure 18. X-ray flux from the superconducting undulator at $1.9 \mathrm{GeV}$, for $30 \mathrm{ps}$ and 200 fs $\mathrm{x}$-ray pulse durations, at $40 \mathrm{kHz}$ (corresponding to the repetition rate of the laser system). Curves are a locus of points from the tuning range of the $1^{\text {st }}$ and $3^{\text {rd }}$ undulator harmonics, plus the wiggler emission (for energies above $\sim 2 \mathrm{keV}$ ). Note that the full beamline flux is at a repetition rate of $500 \mathrm{MHz}$ (scale 30 ps curves by $1.25 \times 10^{4}$ ). 


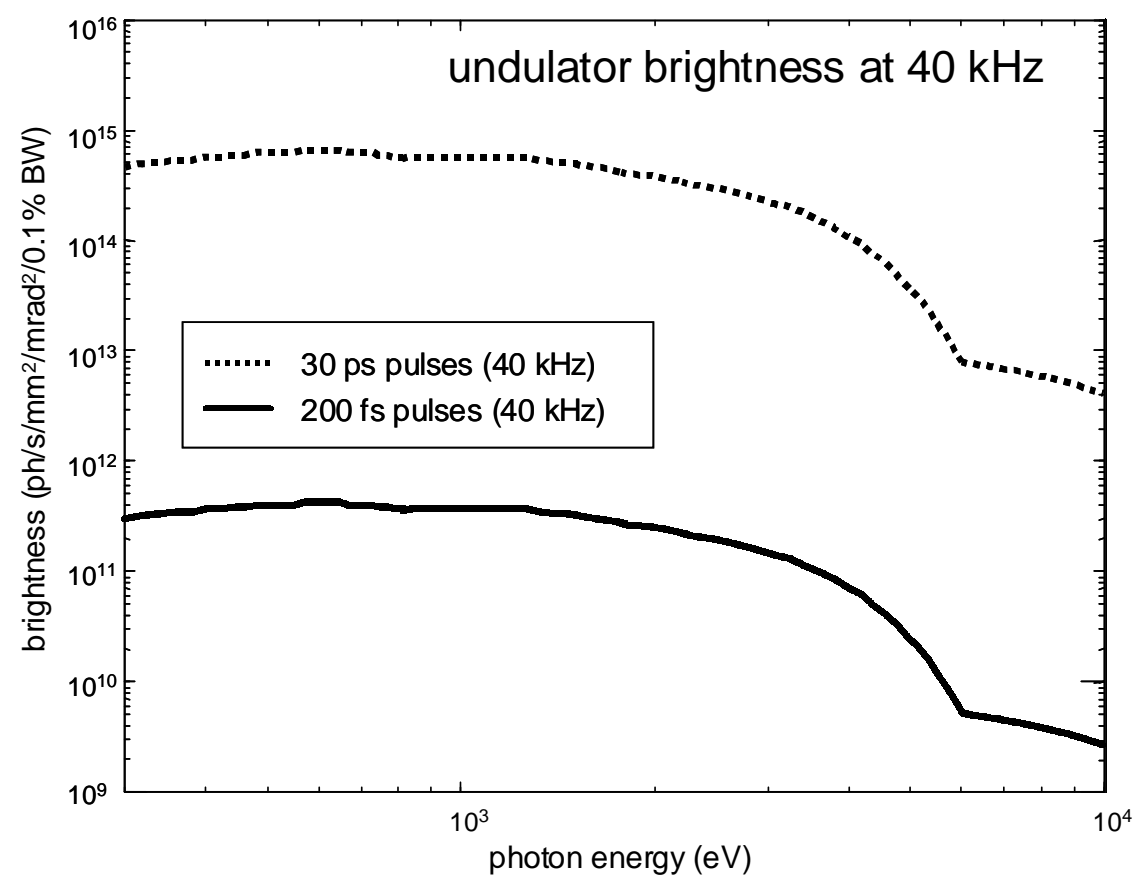

Figure 19. X-ray brightness from the superconducting undulator at $1.9 \mathrm{GeV}$, for $30 \mathrm{ps}$ and $200 \mathrm{fs}$ $\mathrm{x}$-ray pulse durations, at $40 \mathrm{kHz}$ (corresponding to the repetition rate of the laser system). Curves are a locus of points from the tuning range of harmonics 1,3,5,6 and 9, plus the wiggler emission (for energies above $\sim 6 \mathrm{keV}$ ). Note that the full beamline brightness is at a repetition rate of $500 \mathrm{MHz}$ (scale $30 \mathrm{ps}$ curves by $1.25 \times 10^{4}$ ).

\section{Proof-of-Principle Experiments: Femtosecond Synchrotron Pulses from the ALS}

Proof-of-principle experiments have been conducted at the ALS storage ring operating at $\mathrm{E}=1.5 \mathrm{GeV}$ (rms beam energy spread $\sigma_{\mathrm{E}}=1.2 \mathrm{MeV}$ [135]) using a wiggler (W16) with $\mathrm{M}_{\mathrm{W}}=19$ periods, $\lambda_{\mathrm{W}}=16 \mathrm{~cm}$, with the gap adjusted to provide a deflection parameter of $\mathrm{K} \cong 13$. Femtosecond pulses $\left(\tau_{\mathrm{L}}=100 \mathrm{fs}, \mathrm{A}_{\mathrm{L}}=400 \mu \mathrm{J}, \lambda_{\mathrm{L}}=800 \mathrm{~nm}, f_{\mathrm{L}}=1 \mathrm{kHz}\right.$, ) from a Ti:sapphire laser system [136] are synchronized to the storage ring RF using phase-locking techniques [137], and are directed into the main vacuum chamber and co-propagate with the electron beam through the wiggler. Following the interaction region, a mirror directs the fundamental spontaneous wiggler emission and the laser beam out of the storage ring for diagnostic purposes (temporal and spectral overlap, and spatial mode matching between the laser beam and the wiggler emission). The efficiency of the laser/e-beam interaction is tested by measuring the gain experienced by the laser beam passing with the electron beam through the wiggler. The gain is a direct indication of the energy exchange, $\Delta E$, between the laser and the electron beam and is equivalent to the singlepass gain that occurs in free electron lasers [4,29].

The femtosecond time structure of the synchrotron radiation is directly measured using cross-correlation techniques. Visible light ( $2 \mathrm{eV}$ photon energy) from ALS bend-magnet beamline 6.3.2 is imaged onto a nonlinear optical crystal along with a delayed $50 \mathrm{fs}$ pulse from the laser system. Photons at the sum frequency are counted as a function of delay between the modulating laser pulse (propagating through the wiggler) and the laser pulse used for crosscorrelation. An adjustable knife edge located in the beamline at an intermediate image plane of 
the synchrotron radiation (before the nonlinear optical crystal) provides a means to select radiation from different transverse regions of the electron beam.

Figure 20 shows a series of cross-correlation measurements for various knife edge positions. Measurements of the central core of the synchrotron beam (Fig. 20 top) reveal the femtosecond hole or dark pulse that is created due to the acceleration of electrons by the laser pulse. The bright pulse is measured with the knife edge at the $3 \sigma_{x}$ position (Fig. 20 bottom) such that the central core of the beam is blocked. Measurements at $4 \sigma_{\mathrm{x}}$ (not shown) reveal a similar femtosecond synchrotron pulse which is shifted slightly later in time, and demonstrate that the laser pulse provides at least $6 \mathrm{MeV}\left(\Delta \mathrm{x}=4 \sigma_{\mathrm{x}}\right.$ corresponds to $\left.\Delta \mathrm{E}=5 \sigma_{\mathrm{E}}\right)$ energy modulation to a femtosecond slice of the electron bunch.

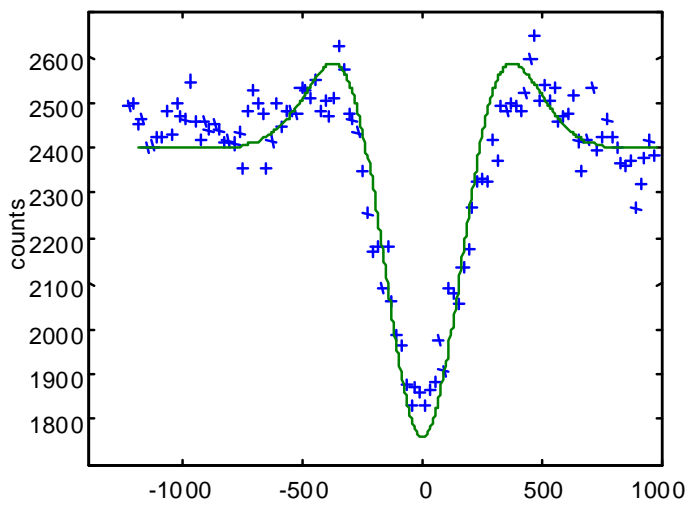

Figure 20. Femtosecond synchrotron pulses measured via cross-correlation between a delayed laser pulse and synchrotron radiation originating from an energy-modulated electron bunch. In (a), synchrotron radiation from the central core $\left(-3 \sigma_{\mathrm{x}}\right.$ to $\left.+3 \sigma_{x}\right)$ of the electron bunch is selected. In (b), synchrotron radiation from the horizontal wings $\left(+3 \sigma_{\mathrm{x}}\right.$ to $\left.+8 \sigma_{\mathrm{x}}\right)$ of the electron bunch is selected. Solid lines are from a model calculation of the spatial and temporal distribution of the energy-modulated electron bunch following propagation through 1.5 arc-sectors

at the

ALS.

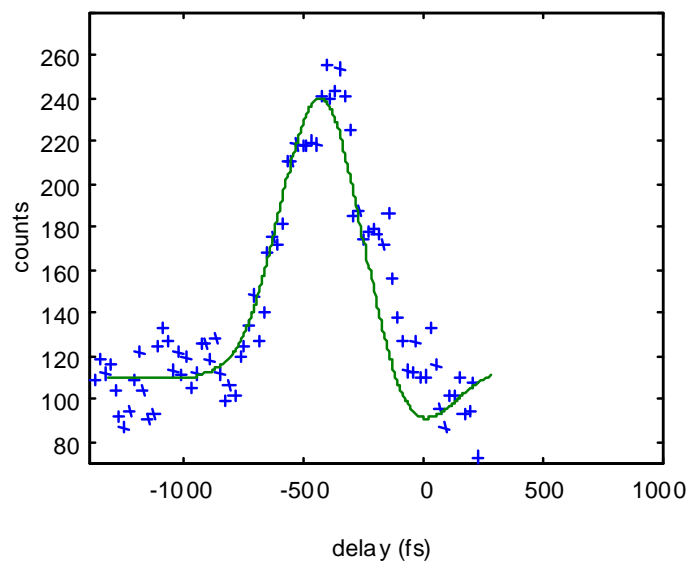

The solid lines in Fig. 20 are based on a model calculation of the electron bunch distribution [4,29]. We additionally account for the measured transverse profile of the visible synchrotron light at the image plane which exhibits non-Gaussian tails beyond $3 \sigma_{\mathrm{x}}$ due to the limited collection aperture of the optics in beamline 6.3.2. This diffraction of visible wavelengths accounts for the relatively large background levels in the measurements shown in Fig. 20 (bottom), but is not an issue at x-ray wavelengths [138]. A peak energy modulation of $\Delta \mathrm{E}=6.4 \mathrm{MeV}$ is assumed and is essentially the only free parameter in the model, aside from an amplitude scaling of the signal. The energy modulation is consistent with that predicted by Eq. 1 using the measured laser pulse energy $\left(A_{L}=400 \mu \mathrm{J}\right)$ and duration at the wiggler $\left(\tau_{\mathrm{L}}=110 \mathrm{fs}\right.$ FWHM), with an interaction efficiency of $\sim 50 \%$ as indicated by the measured gain. The agreement between the theoretical predictions and the experimental measurements (including the 
amplitude of the dark pulse, the time shifts of the bright pulses, and the signal to background ratios) indicates that the model can be confidently applied to predict the characteristics of the femtosecond $\mathrm{x}$-rays and their dependence on experimental conditions. In particular, the location of the bend-magnet beamline used in these demonstration experiments (as shown in Fig. 17) is less than optimum due to the distance (1.5 arc-sectors) from the wiggler in which the laser/e-beam interaction occurs. The UXS facility will collect radiation from the superconducting undulator located one arc-sector after the wiggler. This will reduce the stretching of the electron pulse length due to time-of-flight effects by a factor of 1.5 , resulting in an $\mathrm{x}$-ray pulse duration of $\sim 200 \mathrm{fs}$.

\section{VI.2. Superconducting Undulator}

The UXS facility will rely on a superconducting undulator since such an insertion device can provide the highest possible flux and brightness in the $0.2-10 \mathrm{keV}$ range for the ALS operating at a beam energy of $1.9 \mathrm{GeV}$. An important consideration is that the relatively large vacuum aperture $(12 \mathrm{~mm})$ of this insertion device makes it directly compatible with the current beta functions of the ALS storage ring. This eliminates the need for any modifications to the storage ring lattice. Furthermore, the narrow spectral emission from the undulator lines provide additional means of isolating the femtosecond $\mathrm{x}$-rays by combining spatial separation (as described in section VI.1) with energy separation (as described in section VI.3).

Advances in superconducting magnet technology are beginning to have a major impact on the performance of synchrotron storage rings, with a number of facilities incorporating superconducting insertion devices [139]. The Berkeley Lab has extensive experience in superconducting magnet technology, and the Superconducting Magnet Group is a world leader in this area. The ALS has taken maximum advantage of this expertise as witnessed by the very successful Superbend Project [140]. The Superbend project represents the first time superconducting bend magnets have been introduced into the magnet lattice of a synchrotron light source. The incorporation of these $5 \mathrm{~T}$ bend magnets provides a major performance upgrade for the ALS, and the success of the project is a testament to the effective collaboration between the ALS Accelerator Physics Group, the LBNL Engineering Division, and Wang NMR (the industrial partner responsible for final magnet construction). The superconducting undulator/wiggler will be developed by the same collaborative group that developed the superbends for the ALS.

The radiation source incorporates a $1.5 \mathrm{~m}$ undulator of period length $3 \mathrm{~cm}$. By employing superconducting magnet technology, the device will generate an on-axis peak field of $1.5 \mathrm{~T}$, corresponding to a fundamental wavelength of $116 \mathrm{eV}$, and simultaneously providing useful wiggler emission up to $\sim 10 \mathrm{keV}\left(\mathrm{K}_{\max }=4.2\right)$ in addition to the undulator lines. The undulator will operate with a cold bore with a $12 \mathrm{~mm}$ vacuum chamber aperture and a magnetic gap of $16 \mathrm{~mm}$. A schematic of the undulator is shown in Fig. 21. The undulator itself operates cryogenically at a temperature of $\sim 4 \mathrm{~K}$. Shown in the figure are the $80 \mathrm{~K}$ surfaces as well as the room temperature cryogenic vessel. Space in the vertical and transverse directions amply accommodates superinsulation layering. In the axial direction the shields are positioned optimally so as to accommodate shielding, minimize conduction heat transfer losses, and allow for a smooth RF transition between the insertion device beam vacuum section and the adjacent 
ring vacuum chamber sections. Support for the cryogenic assembly is provided via eight support straps of the type used in the ALS superbend construction [140]. Cooling is provided via two Gifford-McMahon (two-stage) 1.5W cryo-coolers [140].

For the ALS operating at $1.9 \mathrm{GeV}, 400 \mathrm{~mA}$, with emittance parameters $\varepsilon_{\mathrm{x}}=6.75 \times 10^{-9} \mathrm{~m} \cdot \mathrm{rad}$, and $\varepsilon_{\mathrm{y}}=1.5 \times 10^{-10} \mathrm{~m} \cdot \mathrm{rad}$, this undulator will provide a flux of $1 \times 10^{15}$ photons $/ \mathrm{sec} / 0.1 \% \mathrm{BW}$ and a brightness of $8 \times 10^{18}$ photons $/ \mathrm{sec} / \mathrm{mm}^{2} / \mathrm{mrad}^{2} / 0.1 \% \mathrm{BW}$ at a photon energy of $2 \mathrm{keV}$ [141]. The spectral tuning range of this insertion device (flux and brightness at $40 \mathrm{kHz}$ repetition rate) is shown in Figs. 18 and 19 (section VI.1). Note that the maximum flux and brightness from the undulator (500 MHz repetition rate) is a factor of 12,500 higher than the dashed curves shown in Figs. 18 and 19 (which are for $40 \mathrm{kHz}$ operation, corresponding to the laser repetition rate).

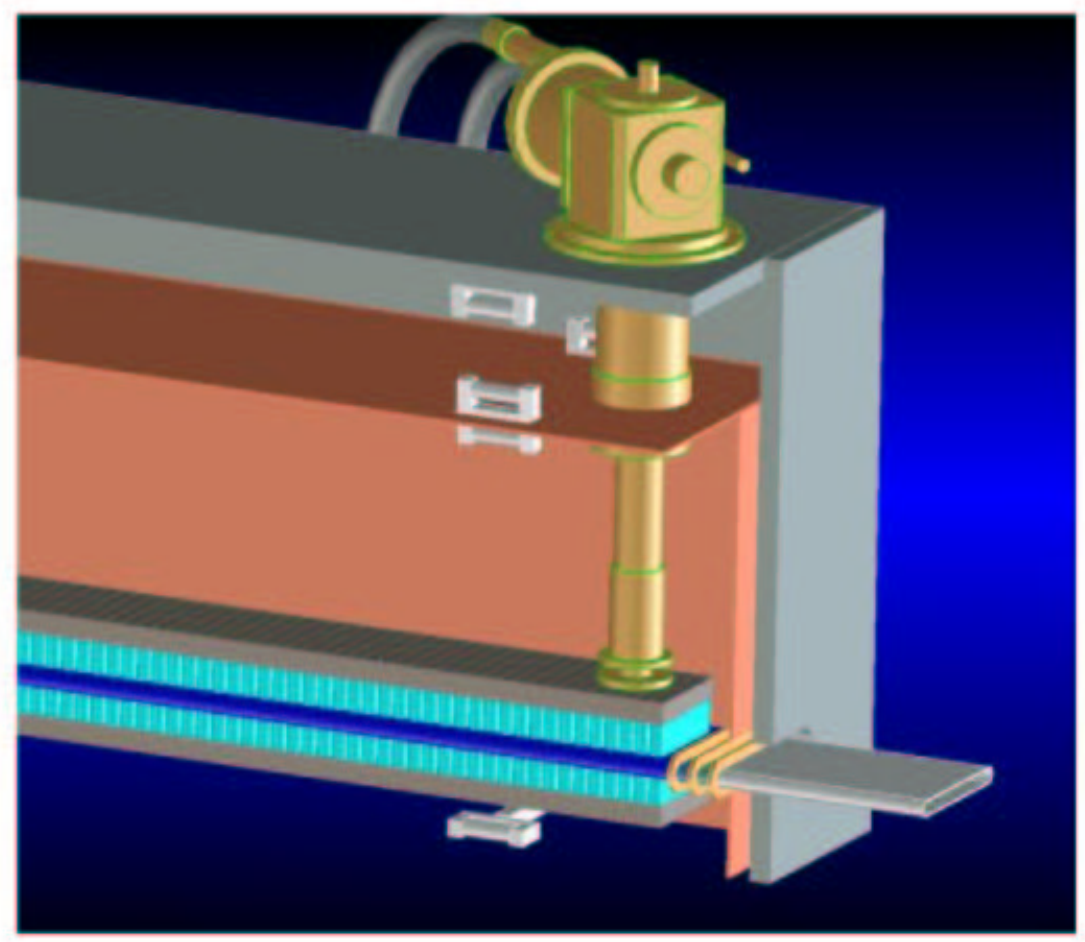

Figure 21. Superconducting undulator design. Room temperature outer chamber is shown in gray, $80 \mathrm{~K}$ surfaces are shown in red. The magnetic coil structure operates at $\sim 4 \mathrm{~K}$ with a magnetic gap of $16 \mathrm{~mm}$. The bore is cold, with a vacuum aperture of $12 \mathrm{~mm}$. Also shown is one of the two-stage cryo-coolers.

The fixed undulator magnetic gap will be $\sim 16 \mathrm{~mm}$, to accommodate the $12 \mathrm{~mm}$ bore vacuum chamber. RF continuity needs to be maintained within the undulator and in the transitions at the undulator ends. The RF transition external to the insertion device will have a maximum slope of 1:10. The upper and lower magnet arrays will be bolted to horizontal support beams and cryogenic contact surfaces, within a cryostat. This entire assembly will be mounted on a stage with standard ALS 6-strut positioning capability. 


\section{VI.3. Beamline Description}

The undulator beamline 6.0.1 will be dedicated for time-resolved x-ray science. It is specifically designed to provide the highest possible flux and brightness over a photon energy range including both hard x-rays and soft x-rays. The beamline incorporates optical components specifically chosen to accommodate femtosecond $\mathrm{x}$-ray pulses by providing low non-specular scattering and high image contrast. The high-average-power, femtosecond laser system will be located at the end of the beamline adjacent to the endstation hutch. The location of beamline 6.0.1 at the straight section immediately following the wiggler minimizes the electron time-offlight contribution to the x-ray pulse duration. Based on the known storage ring parameters and our characterization measurements, we expect that x-ray pulses of $200 \mathrm{fs}$ can be generated from this beamline.

Since the time-resolved x-ray experiments will generally operate at a fraction of the synchrotron bunch repetition frequency $(500 \mathrm{MHz})$, high efficiency in the beamline design is required in order to deliver the maximum possible number of x-ray photons onto the sample. The first consideration is to maximize the laser repetition rate. At a repetition rate of $40 \mathrm{kHz}$ and an average power of $40 \mathrm{~W}$, the laser system for the UXS facility represents a significant advance in the state-of-the-art for femtosecond lasers (see section VI.4). To optimize beamline efficiency, the number of reflections is kept to a minimum. In the soft $x$-ray range, dispersive methods are employed. White beam is imaged onto the sample and subsequently dispersed in a grating spectrometer, thus making use of the full bandwidth of the undulator source.

Another important consideration in the beamline design is the isolation of the femtosecond $\mathrm{x}$-ray pulse from the long-pulse x-ray background originating from unperturbed electrons. The laser pulse modulation of the electron beam energy combined with a vertical dispersion bump in the storage-ring lattice, will cause a fraction of the particles $\left(\sim 10^{-3}\right.$ of the electrons in a bunch) to be vertically displaced within the undulator by a distance of $5 \sigma_{\mathrm{y}}$. A pair of slits at the image plane of the undulator source (displaced $5 \sigma_{\mathrm{y}}$ above the optic axis) will then select the femtosecond $\mathrm{x}$-ray pulses and block the long-pulse background. Previous measurements have verified that the electron beam in the ALS follows a Gaussian profile to $5 \sigma$ [142]. Thus, creating a clean image of the source is critical for minimizing the background. In particular, non-specular scattering in the vertical plane is minimized by using a sagittal focusing mirror. For an x-ray mirror with a grazing incidence angle, in-plane scattering dominates over out-of-plane scattering because of the different length scales of roughness sampled [143]. Measurements of the x-ray beam profile from a similar mirror at ALS beamline 7.3.3 have demonstrated that the out-of-plane scattering can be suppressed by nearly four orders of magnitude compared to the peak specular intensity. This is dominant contribution to the background, and scattering levels in the $10^{-4}$ range or less insures that the integrated femtosecond flux will be larger than the long-pulse background (integrating over one long synchrotron pulse).

An additional method for separating the femtosecond x-ray pulses takes advantage of the relatively narrow spectral bandwidth of the undulator emission combined with the fact that the femtosecond x-rays originate from energy-modulated electrons. Thus the femtosecond x-rays are shifted in energy according to: 


$$
h v=950 \mathrm{n} \mathrm{E}^{2} /\left(1+\mathrm{K}^{2} / 2\right) \lambda_{\mathrm{u}},
$$

where $h v(\mathrm{eV})$ is the undulator harmonic energy, $\mathrm{E}(\mathrm{GeV})$ is the electron beam energy (including the laser-induced modulation), and $\lambda_{\mathrm{u}}(\mathrm{cm})$ is the undulator period. For the third harmonic, the energy separation allows the long-pulse $\mathrm{x}$-ray flux to be suppressed by a factor of $\sim 20$. The two isolation methods based on spatial position and harmonic energy may be applied simultaneously.

In order to take advantage of the entire photon energy range of the undulator source, $0.2-10 \mathrm{keV}$, the beamline is designed to operate alternatively with grating or crystal optics. For $\mathrm{x}$-ray energies greater than $2 \mathrm{keV}$, a pair of crystals will be used in the monochromator. For soft $\mathrm{x}$-ray experiments, the crystals will be translated out of the beam and a plane mirror and plane grating will be employed as a spectrometer for dispersive measurements or as a monochromator. By employing differential pumping instead of a Be window both hard and soft x-rays are transmitted through the same beamline.

Figure 22 shows a schematic diagram of the beamline components, and Table 2 lists the optical elements. The first mirror collects a solid angle of the undulator radiation $1 \mathrm{mrad}$ horizontally by $0.2 \mathrm{mrad}$ vertically. This angular acceptance includes the undulator $2^{\text {nd }}$ harmonic, which helps to cover the photon energy range from 1 to $2 \mathrm{keV}$. The M1 mirror provides a 1:1 image of the source, $500 \mu \mathrm{m}$ horizontal by $70 \mu \mathrm{m}$ vertical. This side-cooled mirror is polished as a cylinder and bent into a toroid. By sagittally imaging the vertical source, the slope error requirement of the mirror is reduced by the grazing angle, $7.5 \mathrm{mrad}$, and so this mirror is relatively easy to manufacture.

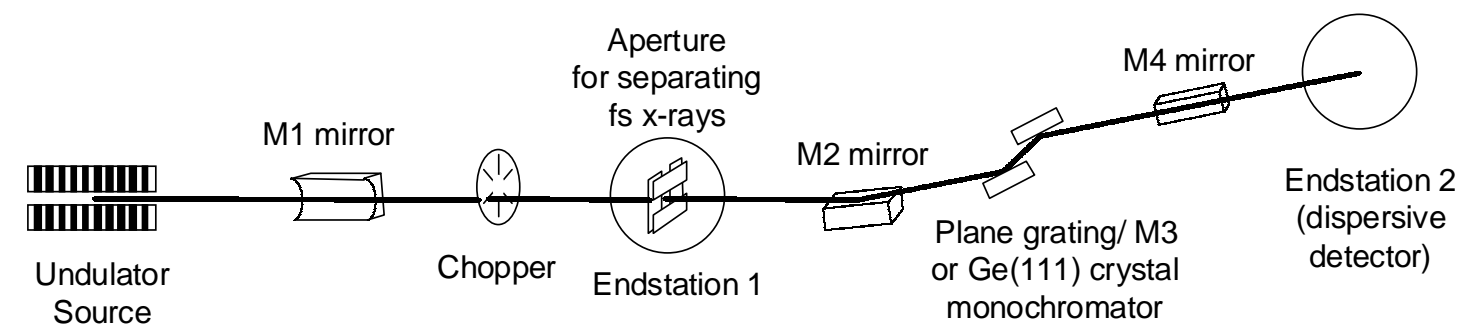

Figure 22. Schematic layout of beamline 6.0.1.

\begin{tabular}{|c|c|c|c|c|c|c|}
\hline & Type & $\begin{array}{c}\text { Coating and } \\
\text { blank material }\end{array}$ & $\begin{array}{c}\text { Dimensions } \\
(\mathrm{mm})\end{array}$ & $\begin{array}{c}\text { Radius } \\
(\mathrm{m})\end{array}$ & $\begin{array}{c}\text { Incidence angle } \\
(\mathrm{deg})\end{array}$ & $\begin{array}{c}\text { Grating period }(\mathrm{mm}) \\
\text { order }\end{array}$ \\
\hline M1 & $\begin{array}{c}\text { toroidal } \\
\text { mirror }\end{array}$ & $\begin{array}{c}\text { Pt-coated } \\
\text { silicon }\end{array}$ & $1200 \times 75$ & $\begin{array}{c}933(\mathrm{R}) \\
0.0525(\rho)\end{array}$ & 89.57 & - \\
\hline M2 & $\begin{array}{c}\text { cylindrical } \\
\text { mirror }\end{array}$ & $\begin{array}{c}\text { Pt-coated } \\
\text { silicon }\end{array}$ & $150 \times 20$ & 356,462 & 89.57 & - \\
\hline M3 & plane & $\begin{array}{c}\text { Pt-coated } \\
\text { silicon }\end{array}$ & $100 \times 20$ & $\infty$ & $85.8-89.1$ & - \\
\hline G1 & $\begin{array}{c}\text { plane } \\
\text { grating }\end{array}$ & $\begin{array}{c}\text { Au-coated } \\
\text { silicon }\end{array}$ & $40 \times 20$ & $\infty$ & $84.5-87.5$ & $-1 / 600$, \\
\hline X1, X2 & crystal & $\begin{array}{c}\text { Germanium } \\
(111)\end{array}$ & $20 \times 20$ & $\infty$ & $7-70\left(\theta_{\mathrm{B}}\right)$ & - \\
\hline M4 & $\begin{array}{c}\text { Plane elliptical } \\
\text { mirror }\end{array}$ & $\begin{array}{c}\text { Pt-coated } \\
\text { silicon }\end{array}$ & $600 \times 65$ & 222 & 89.57 & - \\
\hline
\end{tabular}

Table 2. Optical elements of beamline 6.0.1. 
Following the M1 mirror is an x-ray chopper to match the time-structure (duty cycle) of the x-ray beam to the laser system. The chopper is located at the intermediate focus produced by the M1 mirror. Consequently, the chopper openings (slits) can be narrow enough to provide an opening time of $200 \mathrm{~ns}$. This gate window is short enough to isolate individual $\mathrm{x}$-ray pulses during ALS two-bunch operation. Mechanically chopping single x-ray pulses allows the use of averaging detectors such as CCDs and has been used very successfully at the ESRF for measuring time-resolved Laue diffraction patterns of protein crystals [12,14]. Following the chopper, a pair of slits (as described previously) is used to select the femtosecond x-ray pulses.

The M2 and M4 mirrors relay and demagnify the x-rays from the intermediate focus into the second endstation. In the Kirkpatrick and Baez geometry, the M2 mirror produces a vertical focus at 1:1 magnification while the M4 mirror demagnifies the beam horizontally by 10:1. The M2 mirror is side cooled. Mirrors, similar to the M2 and M4 mirrors, have been successfully developed at the ALS by the bending of initially flat substrates [144]. These methods have worked well for a range of applications including microscopic x-ray diffraction, where a similar mirror system routinely produces a $1 \mu \mathrm{m}$ spot size. For the M2 and M4 mirrors, ray tracing shows a focus of $100 \mu \mathrm{m}$ horizontally by $70 \mu \mathrm{m}$ vertically in endstation 2 .

The monochromator, commercially available from Accel Instruments, will accommodate (in vacuum) both a plane mirror and plane grating, M3 and G1, and a pair of crystals, X1 and X2. $\mathrm{Ge}(111)$ crystals, which have high integrated reflectivity, will be used to monochromatize X-rays with energies above $2 \mathrm{keV}$ with a resolution of $\Delta \lambda / \lambda \approx 1 / 3000$. The M3 mirror and G1 grating comprise a plane grating spectrograph/monochromator. In spectrograph mode the sample is located in endstation 1, either in reflection or transmission geometry, and a multi-channel detector is located in endstation 2. In monochromator mode, endstation 2 will house an exit slit followed closely by the sample. The cylindrical M2 mirror will be bendable to compensate the fixed magnification of the grating. Plane grating monochromators (PGM) have the advantage of covering a wide energy range with a single grating [145]. A spectral resolution of $2.5 \mathrm{eV}$ is calculated at $1450 \mathrm{eV}$ (Al K edge) with a $70 \mu \mathrm{m}$ slit at the intermediate focus. We are currently developing methods to apply grating spectrometry to the entire photon energy range of the undulator, up to $10 \mathrm{keV}$. This will be particularly advantageous for dispersive absorption measurements, by increasing the data acquisition rate.

An important consideration in the design of the beamline is the effect of the beamline optics on the x-ray pulse duration. The most significant contributions to the pulse stretching come from diffractive optics (ruled gratings for example) which introduce wavelength-dependent path lengths, and from Bragg optics in which a number of Bragg planes can contribute to the specular reflection (analogous to optical reflections from multi-stack dielectric mirrors). Stretching of an x-ray pulse upon scattering from a grating arises from the variable path length that is introduced across the profile of the x-ray beam. The maximum path length difference is given by: $\mathrm{m} \cdot \mathrm{N}_{\mathrm{g}} \cdot \lambda_{\mathrm{x} \text {-ray }}$, where $\mathrm{m}$ is the diffraction order and $\mathrm{N}_{\mathrm{g}}$ is the number of illuminated grooves, $N_{g}=D /\left(\theta_{i} \Lambda\right)$, where $D$ is the beam diameter, $\theta_{i}$ is the grazing incident angle, and $\Lambda$ is the groove spacing. In this beamline the contribution of the grating monochromator to the soft $\mathrm{x}$-ray pulse duration is $\sim 30 \mathrm{fs}$. The small magnitude of this effect is a result of the relatively short maximum wavelength of the beamline, and the moderate resolving power of the grating 
monochromator (relatively few illuminated grooves). In the case of Bragg crystals (which are used in the hard $\mathrm{x}$-ray range, $\lambda_{\mathrm{x} \text {-ray }}<6 \AA$ ) the pulse stretching is determined by the path length difference between rays diffracting from the first lattice plane and rays diffracting from the last lattice plane, and is given by $2 \cdot N_{p} \cdot d \cdot \theta_{i}$ where $N_{p}$ is the number of lattice planes contributing to the diffracted beam, and $d$ is the lattice spacing. $N_{p}$ is determined by the effective attenuation length due to dynamical diffraction, and corresponds to the resolving power of the crystal, typically $\$ 3000$. From the Bragg law, $\lambda_{x \text {-ray }}=2 \mathrm{~d} \sin \theta_{i}$., we conclude that two Bragg crystals can generate a total path length difference $\sim 2 \mathrm{~N}_{\mathrm{p}} \lambda_{\mathrm{x} \text {-ray }}$ or $\sim 12$ fs for $2 \mathrm{keV} \mathrm{x}$-rays.

The flux delivered to the sample is calculated for the different operating modes of the beamline and is shown in Fig. 23. The x-ray intensity displayed corresponds to the $200 \mathrm{fs}$ pulse duration at $40 \mathrm{kHz}$ repetition (corresponding to the repetition rate of the laser). The output of the undulator harmonics $1,3,5$ (circles) is evaluated with varying undulator gap ( $\mathrm{K}$ parameter) together with the wiggler radiation (circles). The source intensity is normalized to $0.1 \%$ bandwidth. The crystal monochromator curve at high energies uses the wiggler radiation while the grating monochromator curves use the undulator harmonics. The beamline flux is shown using the Ge(111) crystals (triangles), for the plane grating monochromator (filled squares), and for the plane grating spectrograph (open squares). The reflectivity of the mirrors and the diffraction efficiency of the crystals and gratings are included as appropriate. The $\mathrm{Ge}(111)$ crystal diffraction efficiency is calculated with the program XOP [146]. The grating diffraction efficiencies are calculated from the electromagnetic theory of gratings [147]. Using the grating optics in spectrograph mode enables a large x-ray bandwidth to be detected in parallel. The flux of the undulator beamline is three orders of magnitude higher than that of ALS beamline 5.3.1, the bend magnet beamline presently available for femtosecond x-ray science (see section VII.1 for a detailed performance comparison).

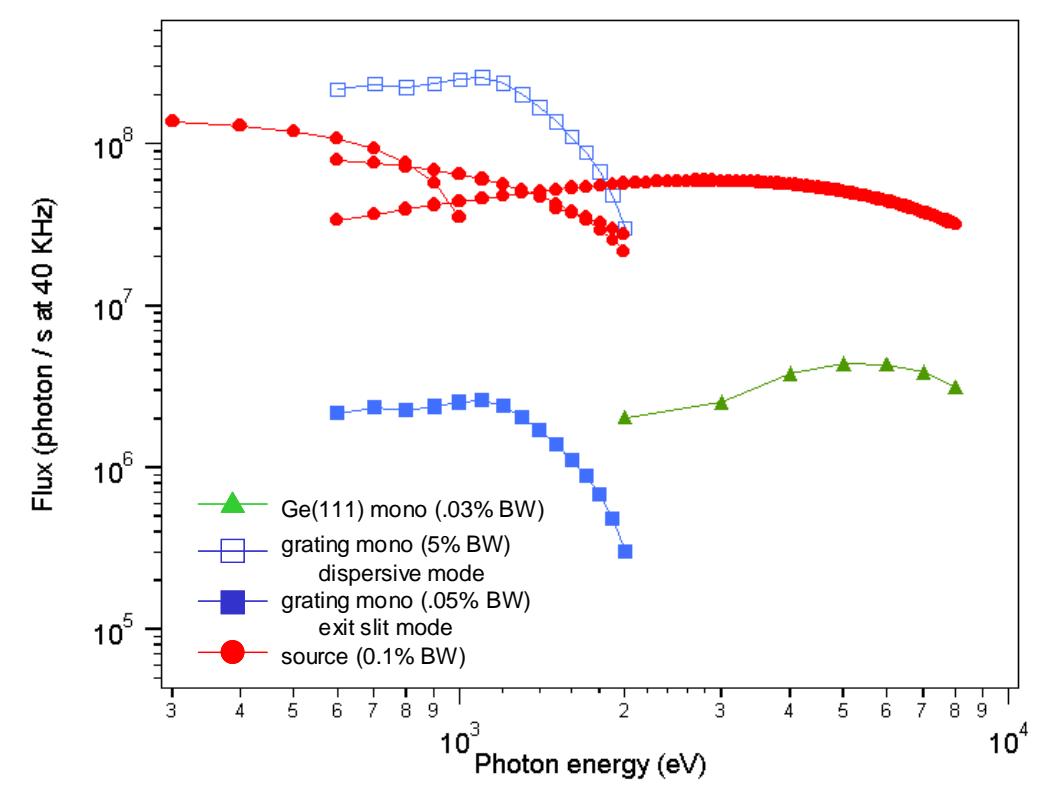

Figure 23. The calculated flux delivered to the sample from beamline 6.0.1 (200 fs pulses, $40 \mathrm{kHz}$ repetition rate). In dispersive mode, the bandwidth is determined by both the spectrograph and the undulator. 


\section{VI.4. Laser System}

The UXS facility represents a complete integration of high-power femtosecond lasers and a $3^{\text {rd }}$ generation synchrotron. The laser system in this facility serves dual purposes, by simultaneously providing (1) ultrashort pulses for modulation (time-slicing) of the relativistic electron beam for subsequent generation of femtosecond x-ray pulses, and (2) suitable ultrashort excitation pulses over the largest possible spectral range for optical excitation of the dynamic process being studied. The technical requirements for the laser system are outlined below:

\section{Laser requirements for electron beam interaction (see VI.1):}

(a) $\sim 1 \mathrm{~mJ}$ pulse energy, $75 \mathrm{fs}$ FWHM, at $\sim 760 \mathrm{~nm}$

(for sufficient energy modulation of the electron beam)

(b) $40 \mathrm{kHz}$ repetition rate, $40 \mathrm{~W}$ average power (average femtosecond $\mathrm{x}$-ray flux scales linearly with repetition rate up to $100 \mathrm{kHz}$ )

(c) diffraction limited focusing, beam parameter: $\mathrm{M}^{2} \leq 1.1$

(for efficient interaction with the electron beam)

\section{Laser excitation pump pulses for time-resolved experiments:}

(a) $\sim 1 \mathrm{~mJ}$ pulse energy for high-field experiments and driving an optical parametric amplifier (at least $1 \mu \mathrm{J}$ pulse energy, tunable over the range from $260 \mathrm{~nm}$ to $\sim 1000 \mathrm{~nm}$ via parametric amplification and frequency mixing techniques)

(b) 75 fs pulse duration, $40 \mathrm{kHz}$ repetition rate

(from common oscillator, synchronized with pulses for electron beam modulation)

(c) $~ 500 \mathrm{~ns}$ delay relative to the pulse used for modulating the electron beam

(to account for propagation delay of the laser pulses as well as the x-rays, see Fig. 1)

\section{System Overview}

The laser system will be based on chirped-pulse-amplification (CPA) [148] in Ti:sapphire (Ti: $\left.\mathrm{Al}_{2} \mathrm{O}_{3}\right)$. The unmatched thermal properties and broad bandwidth of this material allow Ti:sapphire lasers to be scaled to high powers while maintaining excellent beam quality. Ti:sapphire can be efficiently pumped with the second harmonic of solid-state Nd:YAG lasers. Recent development of high-average-power frequency-doubled Nd:YAG lasers now enable Ti:sapphire amplifiers to be scaled to the multi-100 watt level. Pulse compression of highaverage-power sources is made possible by master holographic gratings in which grooves are etched directly into the optical substrate. Such gratings can tolerate power densities of $100 \mathrm{~W} / \mathrm{cm}^{2}$ without beam distortion.

The total average output from the laser system is $80 \mathrm{~W}$ (divided between two $40 \mathrm{~W}$ femtosecond pulse trains). High-power femtosecond laser systems providing $\gtrsim 10 \mathrm{~W}$ of average power have already been demonstrated using Ti:sapphire as the gain medium [149-151], and these techniques can be readily extended to the $40 \mathrm{~W}$ range. The critical components for the laser system are the front-end (oscillator and regenerative preamplifier) and the high-power pump lasers, which are commercial products available from Coherent Inc. (oscillator and pump 
lasers) and from PositiveLight (pre-amplifier). These systems have a proven record of reliability, an important consideration for a user facility.

The block diagram in Fig. 24 illustrates the basic components of the CPA laser system. First, femtosecond pulses from a mode-locked oscillator are stretched using a grating stretcher to reduce the peak power for amplification. Second, the stretched pulses are amplified in a highgain (moderate average power) regenerative amplifier. The pulse is then split, and two individual pulses (one of which is delayed by $\sim 500 \mathrm{~ns}$ ) are amplified in two parallel 2-pass power amplifiers (all operating at $40 \mathrm{kHz}$ repetition rate). The $500 \mathrm{~ns}$ delay line will rely on a multi-pass arrangement between two spherical mirrors, and will incorporate interferometric feedback control to maintain path-length stability. The use of parallel power-amplifiers divides the total laser power, thereby simplifying the thermal loading problem. Finally, each amplified chirped pulse is compressed using a grating compressor.

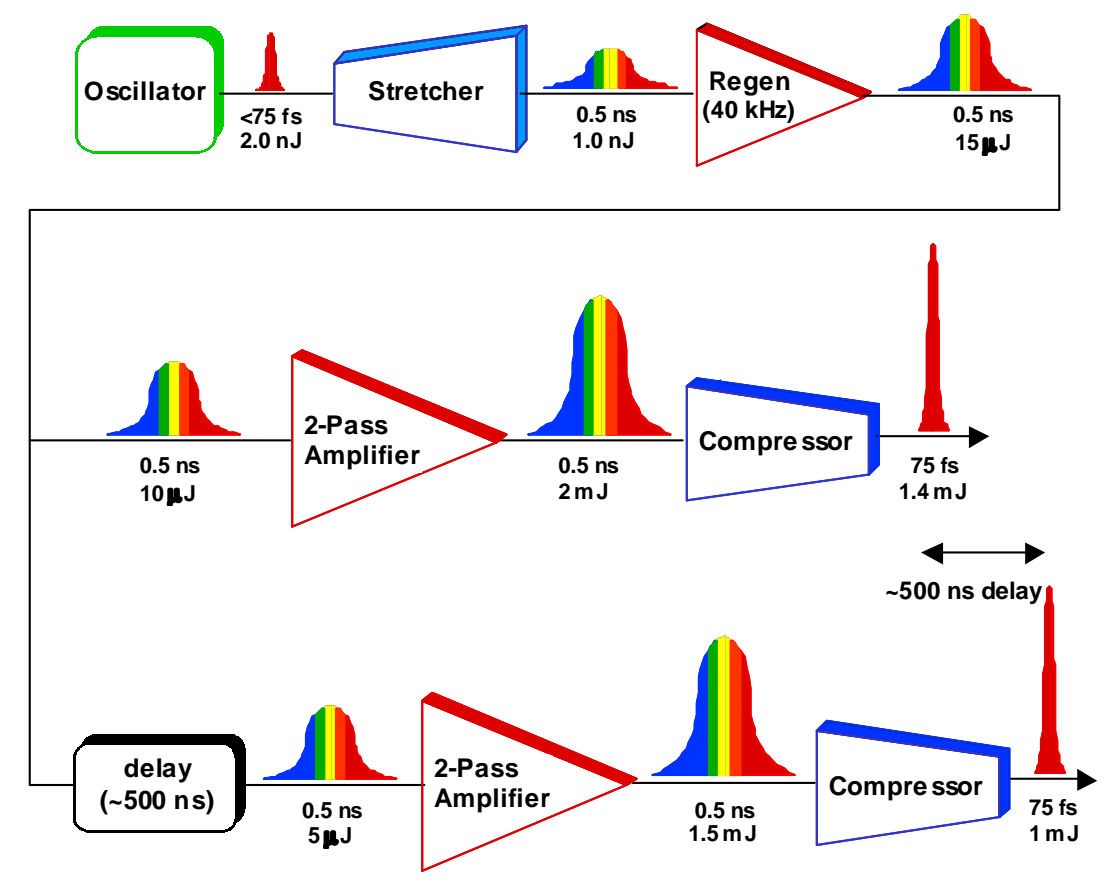

Figure 24. Block diagram of CPA laser system design.

\section{Oscillator/Regenerative Amplifier}

The laser design will employ commercial components where possible to reduce cost and development time and enhance system reliability. Mode-locked Ti:sapphire oscillators suitable for this design are available from several laser vendors including Coherent, Spectra Physics, IMRA America Inc., etc. Experiments at the ALS have already demonstrated the synchronization of such lasers to the storage ring within $\sim 1 \mathrm{ps}[4,15]$. In addition, PositiveLight manufactures a Ti:sapphire regenerative amplifier (Spitfire) that is an established workhorse for ultrafast spectroscopy. This amplifier (pumped by a Q-switched frequency-doubled Nd:YAG laser ) can produce $\sim 15 \mu \mathrm{J}$ pulses (uncompressed) at pulse repetition rates of $40 \mathrm{kHz}$ and will be used as the pre-amplifier. 


\section{Power Amplifier}

The power amplifiers represent the most challenging part of the design due to the high average powers involved. A conceptual design for the power amplifiers is shown in Fig. 25. Each power amplifier consists of two Ti:sapphire rods, which are cryogenically cooled with liquid nitrogen. At $77 \mathrm{~K}$, the thermal conductivity of Ti:sapphire increases by a factor of 30 (to $10 \mathrm{Wcm}^{-1} \mathrm{~K}^{-1}$ ) and the dependence of the refractive index on temperature, $\mathrm{dn} / \mathrm{dT}$, decreases by a factor of 7 (to $1.9 \times 10^{-6} \mathrm{~K}^{-1}$ ) compared to the respective values at $300 \mathrm{~K}$. These effects greatly reduce the thermal gradients in the rod under high pump power conditions and allow Ti:sapphire to be scaled to the 100-watt level without issue. Cryogenic cooling of Ti:sapphire has been pioneered at Lawrence Livermore National Laboratory [152] and at MIT Lincoln Laboratory where cw output powers of $350 \mathrm{~W}$ [153] have been demonstrated. Recently these techniques have been applied to femtosecond amplifiers using chirped-pulse-amplification [150,154-156]. Commercial re-circulating cryogenic chillers with the required capacity for this application are readily available from commercial vendors (Oxford Instruments, IGC-Polycold, etc.). The laser rods are housed in a small vacuum vessel to prevent condensation on the rod surfaces. Each rod is end-pumped with two counter-propagating pump beams. The signal beam enters the amplifier through a polarizer, making two passes through each laser rod. A Faraday rotator in combination with a $\lambda / 2$-waveplate is used to rotate the polarization after the second pass so that the output beam is reflected off of the polarizer. The Faraday rotator allows the signal beam to propagate along the high-gain optical axis of Ti:sapphire during both passes through the amplifier for efficient energy extraction. Also, the rods can be Brewster cut eliminating the need for antireflection coatings, which could be damaged under the high fluences.

The pump lasers for the power amplifiers are diode-pumped, Q-switched, frequency-doubled Nd:YAG lasers with an output power of $\sim 75 \mathrm{~W}$ each at $532 \mathrm{~nm}$. These lasers have recently been developed by Coherent (Corona model) and are based on a simple and robust design. They represent nearly an order of magnitude improvement over previous high-repetition-rate pump lasers. The pump lasers (four for each power amplifier) will operate at a pulse repetition rate of $10 \mathrm{kHz}$, and the pulses are interlaced in time to generate the required $40 \mathrm{kHz}$ repetition rate. Each Ti:sapphire amplifier rod will therefore operate at $20 \mathrm{kHz}$ generating $30-40 \mathrm{~W}$ per rod for a total output power from each amplifier chain of 60 to $80 \mathrm{~W}$ at $40 \mathrm{kHz}$ (before compression). 


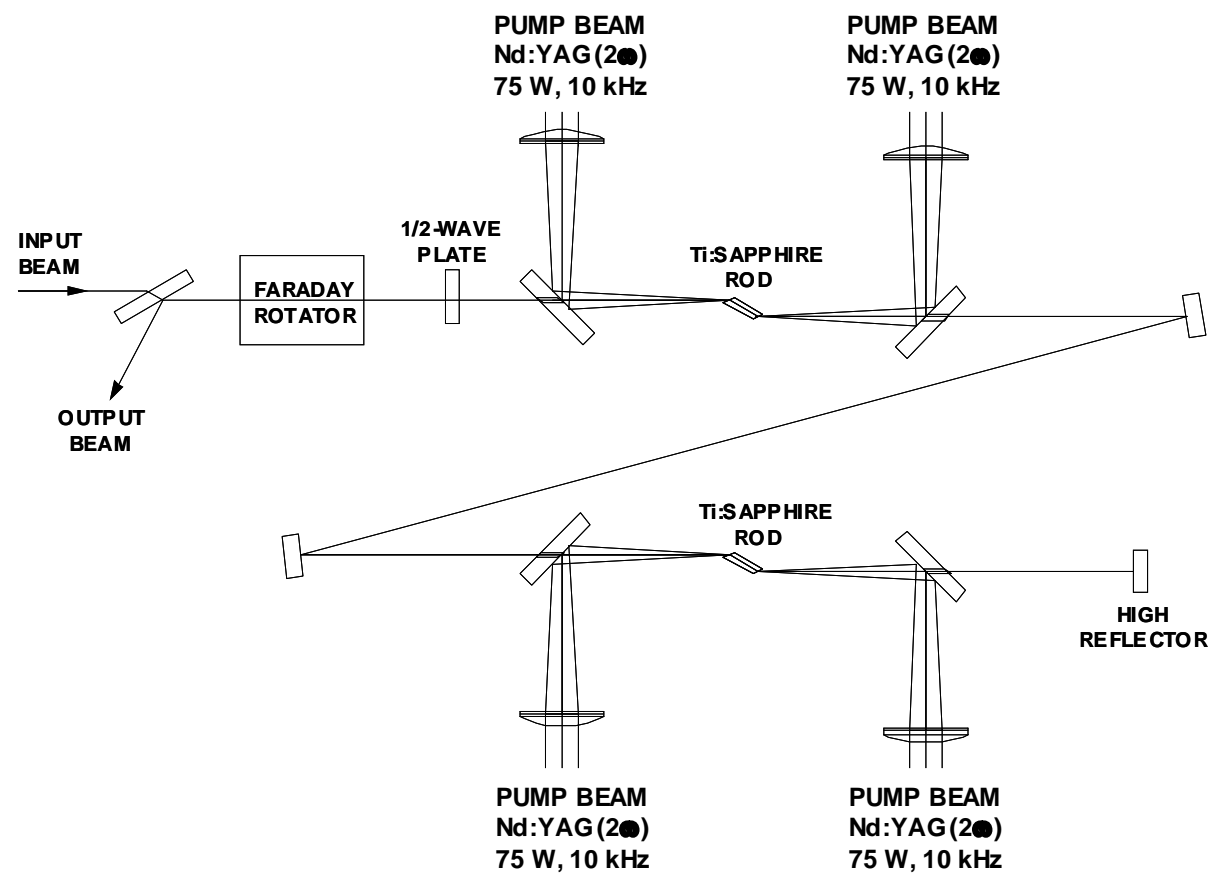

Figure 25. Optical schematic of 2-pass Ti:sapphire power amplifier.

\section{Stretcher/Compressor}

The pulse is stretched and compressed using diffraction gratings in the standard technique developed for CPA systems. High efficiency, master holographic diffraction gratings have been developed by J.-Y. Instruments SA for use in high average power CPA systems. A compressor design using a single grating (to minimize cost and complexity) will be used. The transmission throughput of the compressor is expected to be $\sim 75 \%$, which requires $54 \mathrm{~W}$ from the amplifier to deliver $40 \mathrm{~W}$ out of the compressor.

\section{VI.5. End Station Description}

The two endstations for the UXS beamline are designed to accommodate a variety of time-resolved (and static) x-ray experiments including Bragg and Laue diffraction; photoelectron spectroscopy; and EXAFS and XANES in transmission, reflection, fluorescence, and photoelectron yield modes. The first endstation will consist of a UHV-compatible vacuum chamber with a goniometer and sample manipulator, and will be located at the intermediate focus of the white beam. The second chamber (endstation 2) will be equipped with a 4-axis sample manipulator and will be located following the monochromator. The second chamber will be used for AMO photoelectron spectroscopy and high-field AMO experiments in which the plane-grating and slits serve as a soft x-ray monochromator. This chamber will also house a gated imaging detector for use in dispersive soft x-ray experiments. The endstation will be designed so that either chamber can be easily exchanged for special-purpose chambers that might be required for particular experiments. In addition to the chambers, the endstation-hutch will include an optical parametric amplifier, laser optics, and delay stages for delivering femtosecond excitation pulses at a variety of wavelengths. 
Detectors for the UXS facility are an important consideration. Initially, we will make use of existing commercially-available technology. In the future, detector development will be required to make optimum use of the $\mathrm{x}$-ray flux from the superconducting undulator (e.g. lineararray avalanche photodiode detectors for dispersive EXAFS). Since the repetition rate of the synchrotron is substantially greater than the laser repetition rate, we will make use of high-speed avalanche photodiodes and microchannel plates (MCP's) as detectors for x-rays and photoelectrons in optical-pump/x-ray-probe experiments. The detectors will be gated on the time scale of a few nanoseconds in order to separate the femtosecond $\mathrm{x}$-ray flux (arriving at the laser repetition rate) from the long-pulse x-ray flux (arriving at $500 \mathrm{MHz}$, corresponding to the storage-ring fill pattern). Gating will be accomplished using standard boxcar integrators providing a 2 ns gate width. In addition, the MCP detectors may be pulse-biased either for 1D detection (e.g. dispersive EXAFS) or 2D detection (e.g. Laue diffraction) as part of a gated image intensifier (in combination with a CCD camera). The streak camera described below already incorporates a gated MCP with a phosphor which is coupled to a CCD camera via a fiber bundle. These components can be used independently of the streak tube for gated 1D and 2D detection. Finally, due to the $200 \mathrm{~ns}$ opening time of the beamline chopper, it will also be possible to run experiments without gated detectors during two-bunch or camshaft operation of the storage ring (1.3 $\mathrm{MHz}$ repetition rate).

\section{High-Time-Resolution Streak Camera}

An ultrafast x-ray streak camera is an important end-station component for the UXS facility. The camera will be useful as a diagnostic for the femtosecond $\mathrm{x}$-ray pulses, and can also be put to use in direct detection of $\mathrm{x}$-rays for some time-resolved experiments. The streakcamera will be particularly advantageous for measurements involving the direct detection of $\mathrm{x}$-rays (e.g. Bragg diffraction) in the time range of 1 to $30 \mathrm{ps}$ because it can collect the entire time-evolution of a dynamic process for each 30 ps synchrotron pulse. In the past several years, the use of x-ray streak cameras at $3^{\text {rd }}$ generation synchrotrons has been demonstrated in a number of scientific applications $[3,13,14,16]$. At synchrotron beamlines, streak-cameras operate in integration mode due to the relatively low photon flux per pulse. At present, the timing jitter between successive streak images is one of the major limitations to the achievable temporal resolution.

To meet the requirements of the UXS facility users, we propose to develop a camera operating in the 0.2 to $10 \mathrm{keV}$ range with $\sim 1 \mathrm{ps}$ resolution in integration mode at a repetition rate of $40 \mathrm{kHz}$. This effort will be led by Dr. Zenghu Chang from the Center for Ultrafast Optical Science (CUOS) at the University of Michigan who has considerable previous experience in streak camera development, and their application at synchrotron beamlines.

The camera will be based on a design (shown schematically in Fig. 26) which has already demonstrated 0.5 ps resolution in single-shot operation [157]. The key features of this design are the magnetically-focused streak tube with meander-type deflection plates, and a laser-driven photo-conductive switch for synchronous triggering. The new camera will include improvements in both the streak tube design, the voltage-ramp generator, and the photoconductive switch, which will further enhance the temporal resolution. 


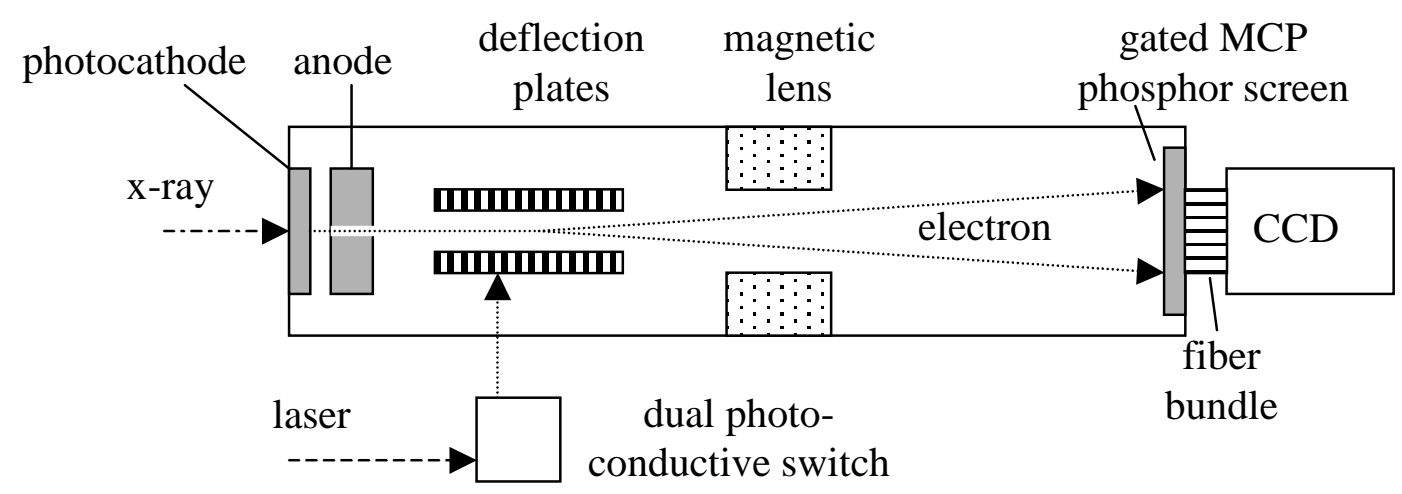

Figure 26. Schematic illustration of the sub-picosecond x-ray streak camera.

\section{Streak Camera Single-shot Temporal Resolution}

The single-shot resolution (presently $\Delta \mathrm{t}_{\mathrm{S}} \sim 0.5 \mathrm{ps}$ ) is determined by three factors, the width of the slit image on the phosphor screen, $w_{S}[\mu \mathrm{m}]$, the deflection sensitivity, $S_{D}[\mu \mathrm{m} / \mathrm{V}]$, and the ramp voltage $\mathrm{K}[\mathrm{V} / \mathrm{sec}]$ :

$$
\Delta \mathrm{t}_{\mathrm{S}}=\mathrm{w}_{\mathrm{S}} /\left(\mathrm{S}_{\mathrm{D}} * \mathrm{~K}\right)
$$

In the present streak-tube design, the width of the slit image is in the range of $80 \mu \mathrm{m}$, as determined by aberrations introduced by the focusing lens and the deflection plates. The deflection sensitivity is $S_{D}=8 \mathrm{~cm} / \mathrm{kV}$. To achieve $\Delta \mathrm{t}_{\mathrm{S}}<0.5$ ps requires a ramp voltage $\mathrm{K}>2 \mathrm{kV} / \mathrm{ns}$. However, the deflection sensitivity is inversely proportional to the electron speed. Since space charge effects are not a constraint for synchrotron beamline applications (due to the relatively low flux per pulse), $S_{\mathrm{D}}$ can be increased to $16 \mathrm{~cm} / \mathrm{kV}$ by reducing the electron speed. In principle this can improve the single-shot resolution by a factor of two using the existing voltage-ramp generator.

\section{Streak Camera Timing Jitter in Integration Mode}

For operation in integration mode, the voltage ramp is generated by a photo-conductive (PC) switch triggered by an optical laser pulse. This approach has demonstrated the lowest timing jitter compared to other voltage-ramp generation schemes. The rise time of the high voltage pulse generated by the PC switch is determined by the frequency bandwidth of the switch design, and thus does not change from laser-pulse to laser-pulse. However, the amplitude of the high voltage pulse does change with the laser pulse energy, and this gives rise to timing jitter which is observed as a spatial smearing in the image plane of the streak camera. The timing jitter due to the voltage-pulse amplitude fluctuations is illustrated in Fig. 27. We assume that $\mathrm{V}_{\mathrm{a}} / 2$ is the voltage to deflect the electrons to the center of the phosphor screen, and $\mathrm{V}_{\mathrm{a}}$ is the average amplitude of the voltage pulses produced by the photo-conductive switch. The timing uncertainty (jitter) is then given by $\Delta \mathrm{t}_{\mathrm{j}}=\mathrm{t}_{2}-\mathrm{t}_{1}=\mathrm{T}_{\mathrm{r}} \cdot \Delta \mathrm{V} /\left(2 \mathrm{~V}_{\mathrm{a}}\right)$ where $\Delta \mathrm{V}=\mathrm{V}_{2}-\mathrm{V}_{1}$ is the shot-to-shot variation of voltage pulse amplitude, and $\mathrm{T}_{\mathrm{r}}$ is the voltage-pulse rise time. 


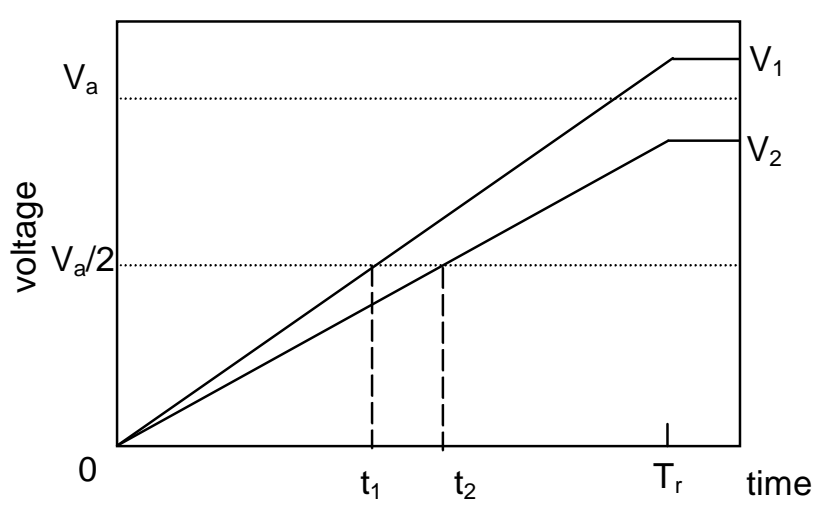

The timing jitter will be reduced by shortening the rise time of the photo-conductive switch. The minimum rise time is limited by the switch design and the response time of the deflection plates. In addition, the rise time should be longer than the desired linear scanning time range of camera. The switch and deflection plates will be redesigned to accommodate a rise time of $\sim 100 \mathrm{ps}$ and a linear scanning range of $\sim 30 \mathrm{ps}$. By operating the switch near saturation, amplitude fluctuations should be suppressed to $\sim 0.5 \%$ which should reduce the timing jitter to less than 1 ps. In addition, we propose to use a novel dual-PC switch that will further minimize the sensitivity to laser energy fluctuation. The streak camera will be enclosed in a separate UHV chamber with independent vacuum pumping so that it can be used at any endstation sample chamber. In addition to the streak tube, high-voltage supply, and PC switch, it also will include a 2 ns gated micro-channel plate image intensifier fiber-coupled to a 16 bit CCD camera. The gated intensifier and camera can be used independently of the streak tube for gated 2D detection of x-rays.

\section{VI.6. Local Vertical Dispersion Bump in the Storage-Ring Lattice}

A superconducting undulator can be installed in straight section 6 of the ALS storage ring without any modifications to the machine lattice. A sizeable dispersion function is necessary to achieve a transverse separation of the energy-modulated electron slice. Because the ALS lattice has zero horizontal dispersion in the straight sections, we will implement a vertical dispersion function. This dispersion can be introduced either at the location of the undulator, or at the location of the neighboring wiggler which is used for energy modulation of electrons, or simultaneously at both locations. The amplitude of the vertical separation of electrons is then given by:

$$
\delta y=\eta_{\text {eff }} \frac{\Delta E}{E_{0}}
$$

where $\Delta E$ is the amplitude of the energy modulation, $E_{0}$ is the electron beam energy and $\eta_{\text {eff }}$ is the effective dispersion function:

$$
\eta_{\text {eff }} \approx \eta_{\text {und }}+\eta_{w} \sqrt{\beta_{\text {und }} / \beta_{w}}
$$


where $\eta_{\text {und }}$ and $\beta_{\text {und }}$ are the vertical dispersion and vertical beta functions (respectively) at the center of the undulator, $\eta_{w}$ and $\beta_{w}$ are the vertical dispersion and beta functions (respectively) at the center of the wiggler.

Measurements of the electron beam profile in the vertical direction at the ALS indicate that it follows a Gaussian distribution up to $\sim 5 \sigma_{y}$, where $\sigma_{y}=\sqrt{\beta_{\text {und }} \kappa \varepsilon_{x}+\left(\eta_{\text {und }} \sigma_{e}\right)^{2}}$, $\kappa=\varepsilon_{y} / \varepsilon_{x}$ is the coupling coefficient, $\varepsilon_{y}$ and $\varepsilon_{x}$ are the vertical and horizontal emittances, and $\sigma_{e}$ is the rms beam energy spread. Beyond $5 \sigma_{y}$, the measured electron distribution drops as $\sim y^{-3}$ [142]. Therefore, the signal-to-background ratio improves only modestly if the separation is increased beyond $5 \sigma_{y}$. On the other hand, larger separation requires increasing the vertical dispersion in the lattice and/or increasing the laser pulse energy. A model calculation using $\varepsilon_{x}=4 \times 10^{-9} \mathrm{~m} \cdot \mathrm{rad}, E_{0}=1.9 \mathrm{GeV}$, and $\sigma_{e}=10^{-3}, \kappa=0.01$ and a laser pulse energy of $600 \mu \mathrm{J}$ (with the laser pulse bandwidth being matched to the bandwidth of the spontaneous wiggler emission at the third harmonic, following Eq. $\left.2, \mathrm{M}_{\mathrm{L}} \cong 2.1 \cdot \mathrm{M}_{\mathrm{W}}\right)$ gives $5 \sigma_{y}$ separation at a value of $\eta_{\text {eff }} \cong 20 \mathrm{~mm}$. It is clear that the small vertical emittance allows us to use a smaller vertical dispersion to achieve the same separation of electrons.

Two methods have been studied to generate $\eta_{\text {eff }}$. The first method creates a local $\eta_{y}$ bump by coupling the horizontal dispersion into the vertical plane. The second method generates a $\eta_{y}$ wave in the ring using a localized vertical orbit bump. Skew quadrupoles can be used to transform horizontal dispersion into the vertical plane in the same way that a corrector magnet steers a closed orbit. At the ALS, the existing sextupole magnets are equipped with special coils that produce a skew quadrupole field. Eight such sextupoles are available in two arcs surrounding the wiggler. All of them can be used to produce the required $\eta_{\text {eff }}$. Useful vertical dispersion can also be created using a vertical orbit bump localized around the undulator. This orbit bump is confined primarily to a region of the lattice without sextupole magnets; thus it is free from the non-linear lattice effects. The vertical dispersion produced in this way can further be used to compensate the unwanted $\eta_{\text {eff }}^{\prime}$ in the undulator which appears due to existing spurious vertical dispersion in the ring. Reference [158] contains a detailed description of both techniques.

\section{Background and Comparison with Existing Femtosecond X-ray Sources}

\section{VII.1. Femtosecond Bend-magnet Beamline at the ALS}

This proposal builds on considerable expertise in the generation of femtosecond x-rays and in time-resolved x-ray spectroscopy developed at the ALS and at U.C. Berkeley. The generation of femtosecond $\mathrm{x}$-rays via laser/electron beam interaction was pioneered by Schoenlein and Leemans using Thomson scattering with the ALS linac injector [15,48]. The application of ultrafast $\mathrm{x}$-ray streak cameras using synchrotron sources was pioneered at the ALS 
by the Falcone group [13]. These efforts resulted in the initial ultrafast spectroscopy experiments which used time-resolved diffraction techniques to study laser-induced disordering in crystals [3,9]. The more compelling research applications in ultrafast $\mathrm{x}$-ray science clearly require substantial enhancements in $\mathrm{x}$-ray flux, and continued development of ultrafast $\mathrm{x}$-ray techniques.

To date, time-resolved experiments at the ALS have been done during independent investigator time on bend-magnet beamlines that were designed primarily for other purposes. For example, the streak-camera based experiments described in section IV have been done on beamline 7.3.3 which is primarily for x-ray micro-diffraction research. Similarly, the initial experiments demonstrating the generation of femtosecond synchrotron radiation (section VI.1) have been done on beamline 6.3.2, which is designed for EUV reflectometry (and is one of the most heavily-subscribed beamline at the ALS). The limited available beamtime, and non-ideal beamline performance has considerably restricted the progress of time-resolved x-ray research. Thus there is a strong and immediate need for a dedicated beamline which is optimally designed for ultrafast $\mathrm{x}$-ray science. The superconducting undulator beamline which is the subject of this proposal, represents the practical performance limit of what can be achieved in femtosecond x-ray flux and brightness from the ALS using the techniques described in section VI.1. However, the total development period (from initial concept to beamline commissioning) of a project of this scale is quite long, typically 4-5 years.

As an interim solution, a bend-magnet beamline (BL 5.3.1) dedicated to time-resolved $\mathrm{X}$-ray science has recently been constructed at the ALS. This beamline serves as the current home for time-resolved x-ray research, and has been designed specifically to enable the novel technique for generating femtosecond synchrotron radiation to progress beyond the proof-ofprinciple stage to actual scientific applications. Beamline 5.3.1 is relatively simple, consisting of a single toroidal mirror which images the bend-magnet source onto a pair of slits for selecting the femtosecond synchrotron radiation. The beamline also includes a double-crystal monochromator which provides a bandwidth of $\lambda / \Delta \lambda \sim 3000$. The beamline was constructed with a modest amount of seed money: $\sim 300 \mathrm{~K}$ in capital equipment money from the University of California (UCDRD) and a matching contribution from the ALS provided in the form of design, engineering, and installation support. A Ti:sapphire laser system operating at $5 \mathrm{kHz}$ and synchronized to the storage ring is available at this beamline for femtosecond modulation of the electron beam. The beamline was constructed in one year, and represents a 100x improvement in performance (average x-ray flux and brightness) in comparison with the previous femtosecond X-ray source at the ALS (based on Thomson scattering with the ALS linac injector) [29]. Nevertheless, beamline 5.3.1 is intended to be an interim solution during the search for funding and eventual construction of the undulator-based beamline which is the subject of this proposal.

The research program described in section IV (and the feasibility estimates in the proposal appendix) illustrate the demanding x-ray flux and brightness requirements for timeresolved experiments. Although the capabilities of the bend-magnet beamline are sufficient for time-resolved Bragg diffraction and some EXAFS measurements in solid-density materials, it is clear that the beamline flux is not nearly sufficient for the wide range of scientific applications of time-resolved $x$-ray research as described in this proposal. Figure 28 compares the performance of the existing bend-magnet beamline with the proposed femtosecond undulator beamline. The 
undulator provides a 100x improvement in flux and a 1000x improvement in brightness in comparison with a bend magnet source. Furthermore, the increase in the laser repetition rate provides an additional 10x increase in both flux and brightness for time-resolved experiments. Thus the data acquisition times estimated in the appendix would be $\sim 1000$ times longer on the bend-magnet beamline. Finally, expected developments in commercial laser technology, particularly Nd:YAG pump lasers, should in the future provide an additional factor of $2.5 \mathrm{x}$ enhancement by allowing operation at $100 \mathrm{kHz}$ repetition rate.

The overall enhancement factors (flux: $10^{3}$, and brightness: $10^{4}$ ) apply to pump-probe experiments on the picosecond time scale (using the full duration of the electron bunch and/or the ultrafast x-ray streak camera) and also to pump-probe experiments on the femtosecond time scale using x-rays from laser-modulated bunches. Such performance is near the practical limit of what can be achieved from an existing third-generation synchrotron, without substantial modifications to the storage-ring lattice and operation. Beamline performance at this level is absolutely essential for the most compelling scientific experiments in ultrafast $\mathrm{x}$-ray spectroscopy.

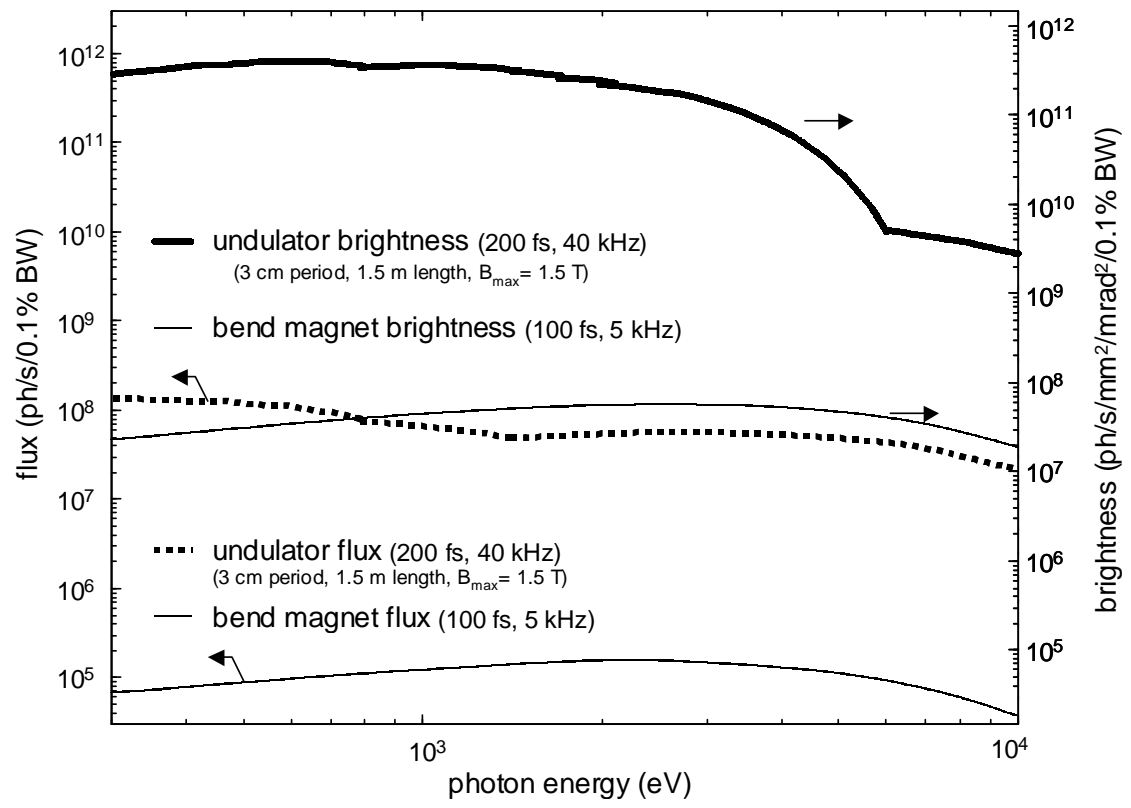

Figure 28. Average femtosecond flux and brightness from the proposed undulator beamline compared with that of the existing bend-magnet beamline. The undulator curves are a locus of points from the tuning range of the odd harmonics, plus the wiggler emission.

\section{VII.2. Other Sources of Femtosecond X-rays}

In the past decade, important advances have been made in the generation of femtosecond $\mathrm{x}$-ray pulses using a variety of techniques including: high-order harmonic generation from femtosecond optical pulses [23-26], laser-plasma based x-ray sources [11,18,27], laser-driven $\mathrm{x}$-ray diodes [17], and femtosecond x-rays from Thomson scattering [15]. While initial timeresolved x-ray experiments (with modest source requirements) have been performed using these sources, they suffer from significant limitations in one or more critical parameters including: (a) 
x-ray average brightness and/or flux, (b) x-ray photon energy, tunability, and spectral range, and (c) x-ray pulse duration and control. As a consequence, these sources are not able to meet the demanding requirements of the scientific program outlined in section IV. Here we briefly consider the limitations of short-pulse x-ray sources that are currently available for time-resolved research.

High-order harmonic generation from femtosecond optical pulses has advanced significantly in recent years. Laser harmonics offer the advantage of temporal and spatial coherence, and potentially sub-femtosecond pulse durations. To date laser harmonics have been generated up to $550 \mathrm{eV}[24,25]$, with a yield of a few photons per pulse at the highest photon energies. Recently, phase-matching techniques have been demonstrated to increase the harmonic yield for photon energies up to $70 \mathrm{eV}$ [26]. While this is an active research area in source development, current harmonic-generation schemes are not likely to yield sufficient flux in the 1$10 \mathrm{keV}$ range for demanding $\mathrm{x}$-ray spectroscopy applications.

Laser-plasma based x-ray sources can provide high x-ray flux [11,27], and a time resolution of $<600 \mathrm{fs}$ has been achieved by carefully controlling the excitation conditions [27]. However, plasma-based sources are inherently low-brightness since $\mathrm{x}$-rays are emitted in a $4 \pi$ solid angle. This is a significant disadvantage for experiments requiring limited source divergence (such as Bragg diffraction) and presents a problem for designing optics with reasonable collection efficiency. One current approach is to use bent crystal optics [27], which can collect a large solid angle, but are effective only over very narrow bandwidths. Finally, plasma-based sources are not easily tunable since the highest flux comes from the line emission $\left(\mathrm{K}_{\alpha}\right.$ radiation) [159].

Laser driven x-ray diodes (essentially $x$-ray tubes with photocathodes illuminated by femtosecond laser pulses) are also being developed for time-resolved x-ray research [7]. The properties of these sources are similar to the laser-plasma sources in that x-rays are emitted in $4 \pi$, and the spectrum consists of line emission superimposed on a continuum background. Adjusting the electron energy provides some means of control over the x-ray spectrum, and the $\mathrm{x}$-ray pulse duration is determined by the electron bunch duration. However, the $\mathrm{x}$-ray flux is determined by the charge in the electron pulse, which is limited by space-charge effects. Spacecharge effects also limit the achievable electron pulse duration, which is typically in the picosecond range [7].

In a related approach, electron pulses from laser-driven photocathodes have been directly used to probe structural dynamics via electron diffraction [160-162]. The electron penetration depth limits the applicability of this approach to gas phase (or thin-film) samples. The available time resolution ( $2 \mathrm{ps}$ ) is limited by the achievable electron bunch duration (mediated by spacecharge effects) as well as the velocity mismatch between photon pump-pulses and nonrelativistic electron probe-pulses. Finally, electron interactions with matter do not provide the same element-specificity as $\mathrm{x}$-ray interactions.

Finally, the generation of femtosecond x-rays via Thomson scattering between relativistic electrons and terawatt laser pulses has been pioneered at the ALS. This approach offers some advantages for generating femtosecond $\mathrm{x}$-rays with well-controlled pulse duration in a directed 
beam [15]. However, the flux from such sources is presently limited by the electron-beam current and the average laser power, while the brightness is limited by the emittance of the electron beam. Furthermore, the scattering geometry required for achieving femtosecond x-ray pulse durations also imposes fundamental limits on the flux and brightness that can be generated via Thomson scattering [29]. Such limitations are particularly significant at low photon energies ( $\sim 10 \mathrm{keV}$ or less) and for short pulse durations, $\sim 100 \mathrm{fs}$ [29]. Finally, it is important to note that even though the proposed undulator beamline operates at a high repetition rate, the flux per pulse (on sample) is comparable to what one might expect from an optimized $90^{\circ}$ Thomson scattering source operating at much lower repetition rates. See ref. [29] for a detailed comparison.

\section{Budget, Administration, and Management}

\section{VIII.1. Budget and Budget Justification}

\section{Cost Sharing}

Other funding agencies have expressed interest in funding components of this project. The beamline proposal was submitted to the NSF MRI Program in February 2001 (R.W. Falcone-P.I.), with a specific request for funds for the laser system. The proposal was favorably reviewed, and NSF offered to contribute $\$ 0.5 \mathrm{M}$ toward the laser system (contingent on a decision by DOE to proceed with the project). These funds were to be matched with an additional $\$ 0.5 \mathrm{M}$ in non-federal money from the University of California. The development of the streak camera is now being supported through a grant to R.W. Falcone from DOE Defense Programs.

In-kind support is also provided by the ALS for several components of this project. First, the availability of the existing wiggler (W16) which is necessary for laser-modulation of the electron beam represents a substantial cost savings $(\$ 2-3 \mathrm{M})$ for this project. The ALS is presently funding modifications to the wiggler to facilitate simultaneous operation for both the UXS facility and the protein crystallography beamline. Second, ALS personnel (P. Heimann and T. Glover) from the Experimental Systems Group have been continuously involved in the development and demonstration experiments for generating femtosecond x-rays from the storage ring ( $\$ 500 \mathrm{~K}$ cost over the past three years). Finally, critical technical support from the ALS Accelerator Physics Group is provided by the ALS. Due to the unique nature of this project, Accelerator Physics support is substantially beyond what is typically required for other synchrotron beamlines.

Options for generating additional support from other funding agencies for this project are currently being explored. The preferred approach is to develop a strategy with guidance from BES on how best to proceed in this area. Both NSF (through the Major Research Initiative Grant Program) and DOE Defense Programs (through the High Energy Density Research Program) are currently supporting (or have supported in the recent past) the research activities of various core research team members, and this research will be substantially enhanced by the capabilities of 
the UXS facility. Beyond this, there is potential interest from DOE Defense Programs related to instrumentation diagnostics for the National Ignition Facility. For example, the combination of ultrafast time resolution, tunability, and high photon energy make the UXS facility uniquely capable of characterizing the ultrafast detectors that will be developed for use in NIF experiments.

\section{Financial Plan Summary and Explanation}

The UXS Facility will occupy the Sector 6 straight section at the ALS. The construction costs for the UXS Facility undulator, beamlines and laser system are presented in Table 3. The UXS Facility financial plan has been prepared with and approved by the ALS office for project management and budget.

\begin{tabular}{|l|r|r|r|r|r|r|r|}
\hline & $\begin{array}{c}\text { Labor } \\
2002\end{array}$ & $\begin{array}{c}\text { Equipment } \\
2002\end{array}$ & $\begin{array}{c}\text { Labor } \\
2003\end{array}$ & $\begin{array}{c}\text { Equipment } \\
2003\end{array}$ & $\begin{array}{c}\text { Labor } \\
2004\end{array}$ & $\begin{array}{c}\text { Equipment } \\
2004\end{array}$ & $\begin{array}{c}\text { System } \\
\text { Totals }\end{array}$ \\
\hline Undulator & $\mathbf{0}$ & $\mathbf{0}$ & $\mathbf{3 9 0}$ & $\mathbf{2 3 0}$ & $\mathbf{3 0 5}$ & $\mathbf{2 8 0}$ & $\mathbf{1 , 2 0 5}$ \\
\hline $\begin{array}{l}\text { vacuum } \\
\text { chamber }\end{array}$ & 0 & 0 & 150 & 0 & 65 & 165 & \\
\hline $\begin{array}{l}\text { magnetic } \\
\text { structure }\end{array}$ & 0 & 0 & 120 & 230 & 180 & 0 & \\
\hline $\begin{array}{l}\text { support } \\
\text { and drive }\end{array}$ & 0 & 0 & 120 & 0 & 60 & 115 & \\
\hline Front End & $\mathbf{0}$ & $\mathbf{0}$ & $\mathbf{1 7 2}$ & $\mathbf{3 3 5}$ & $\mathbf{0}$ & $\mathbf{0}$ & $\mathbf{5 0 7}$ \\
\hline Beamline & $\mathbf{1 3 5}$ & $\mathbf{6 4 0}$ & $\mathbf{4 3 5}$ & $\mathbf{2 7 5}$ & $\mathbf{3 0 0}$ & $\mathbf{3 5 0}$ & $\mathbf{2 , 1 3 5}$ \\
\hline optics & 10 & 200 & 0 & 0 & 0 & 0 & \\
\hline crystal mono & 10 & 440 & 0 & 0 & 0 & 0 & \\
\hline $\begin{array}{l}\text { mirror, grating } \\
\text { systems }\end{array}$ & 115 & 0 & 435 & 275 & 300 & 350 & \\
\hline Endstations & $\mathbf{0}$ & $\mathbf{0}$ & $\mathbf{0}$ & $\mathbf{4 3 7}$ & $\mathbf{0}$ & $\mathbf{0}$ & $\mathbf{4 3 7}$ \\
\hline Laser System & $\mathbf{1 2 3}$ & $\mathbf{1 2 2 5}$ & $\mathbf{1 5 4}$ & $\mathbf{7 8 2}$ & $\mathbf{3 1}$ & $\mathbf{2 5}$ & $\mathbf{2 , 3 4 0}$ \\
\hline front end amp & 0 & 390 & 0 & 0 & 0 & 0 & \\
\hline pump lasers & 0 & 590 & 0 & 588 & 0 & 0 & \\
\hline power amps & 123 & 170 & 124 & 169 & 0 & 0 & \\
\hline elect. and mech. & 0 & 75 & 30 & 25 & 31 & 25 & \\
\hline $\begin{array}{l}\text { Project } \\
\text { Management }\end{array}$ & $\mathbf{5 2}$ & $\mathbf{0}$ & $\mathbf{1 0 4}$ & $\mathbf{0}$ & $\mathbf{7 8}$ & $\mathbf{0}$ & $\mathbf{2 3 4}$ \\
\hline Subtotals & 310 & 1,865 & 1,255 & 2,059 & 714 & 655 & \\
\hline Escalation & $\mathbf{0}$ & $\mathbf{0}$ & $\mathbf{4 6}$ & $\mathbf{4 1}$ & $\mathbf{2 7}$ & $\mathbf{1 4}$ & $\mathbf{1 2 8}$ \\
\hline Yearly Totals & $\mathbf{2 , 1 7 5}$ & $\mathbf{3 , 4 0 1}$ & & $\mathbf{1 , 4 1 0}$ & $\mathbf{6 , 9 8 6}$ \\
\hline
\end{tabular}

Table 3. Ultrafast X-ray Science facility construction costs over 3 year period in $\mathrm{K} \$$. LBNL overheads and contingency are included. Escalation rates are included at $2.0 \%$ per year for equipment and $3.7 \%$ per year for labor 


\section{Undulator}

The undulator cost was estimated based on LBNL fabrication costs and overheads. Significant cost savings have been achieved by adopting a design based on superconducting magnets with an open aperture that is consistent with the existing ALS storage ring parameters. This further eliminates the need for any modifications to the storage ring lattice. Contingency was included at $30 \%$.

\section{Modifications to Storage Ring Lattice}

Storage ring modifications are no longer required. The new undulator design is not a small-aperture device and therefore does not require changes to the beta function of the ALS storage ring lattice.

\section{Front End}

The front end is the same as that used for beamline 10.0.1. Thus the actual costs are known, and there are no changes to the design other than the dimensions of apertures. The contingency is included at $15 \%$.

\section{Beamline}

The numbers for these items have been estimated by P. Heimann in consultation with ALS beamline engineers. The fact that the beamline contains a novel monochromator including crystal-optics as well as grating-optics is reflected in the overall cost. Existing designs as well as commercially available components will be used when possible. Contingency was included at $10 \%$ for all components and equipment, with $30 \%$ contingency only for labor costs.

\section{End Station}

The cost of the end station is generally based on the costs of comparable end station and detectors being developed for beamline 5.3.1. The costs include the chambers, sample manipulators and detectors. The electron spectrometer requested in the original budget has been eliminated. In addition, the streak camera has been eliminated from the original budget as we have identified other sources of funding for this. Contingency was included at $15 \%$.

\section{Laser System}

The laser system design has been modified to incorporate less expensive components that are commercially available. The cost of the laser system is based on known costs of the commercial components which comprise the bulk of the system (75\% of the total cost). In addition, the costs of the optical components and mounts, diffraction gratings, and cryogenic chillers are based on quotations from commercial vendors. System integration and fabrication costs for custom components (such as vacuum chambers) have been estimated by LBNL Engineering Division. Contingency is included at 30\%, with $10 \%$ contingency on the capital equipment components.

\section{Operations}

Due to the unique nature of the UXS facility, commissioning will be done by the original group which developed and demonstrated the technique for generating femtosecond $\mathrm{x}$-rays from 
the ALS storage ring. This group includes ALS Experimental Systems Group staff: P. Heimann and T. Glover.

Ongoing operational support for the UXS facility including beamline personnel and materials and supplies will be requested in a separate proposal to DOE Basic Energy Sciences. Due to the complexity of the UXS facility, the requirements for ongoing operational support are anticipated to be more extensive than for typical beamlines. For the UXS facility to function at peak performance, several complex systems need to operate simultaneously including the highaverage-power laser system, ultrafast x-ray streak camera and gated detectors, superconducting undulator etc. At this time, we estimate that a minimum of $\sim 1.5 \mathrm{FTE}$ will be required.

\section{Construction Schedule}

This proposal for the UXS Facility has been structured around a three year construction schedule. This schedule has been determined by the time needed to construct the undulator and takes into account the existing and anticipated demands for ALS engineering resources by a variety of other ALS projects.

\section{VIII.2. Organization and Management}

Ultrafast $\mathrm{x}$-ray science is an emerging field of research which to date has been based largely on laboratory-scale, short-pulse x-ray sources and femtosecond lasers. This initial developmental work has brought the field to a critical point where the scientific applications will flourish only if the source capabilities can be substantially improved. Thus, the availability of a femtosecond synchrotron beamline with flux and brightness substantially beyond laboratoryscale sources will be instrumental in advancing this field.

To take maximum advantage of the opportunities afforded by the UXS facility, we have assembled a scientific research team which will form the nucleus of a new user community for ultrafast $\mathrm{x}$-ray science at a $3^{\text {rd }}$ generation synchrotron (and in the future at a $4^{\text {th }}$ generation light source). This group has scientific expertise in several critical areas including:

(a) X-ray research at synchrotron sources including diffraction, EXAFS, and XPS applied to crystalline solids, nanostructures, molecules, and atoms.

(b) optical ultrafast spectroscopy in condensed matter, molecular, and atomic systems

(c) time-resolved $\mathrm{x}$-ray spectroscopy using femtosecond lasers

The scientific expertise is complemented by technical expertise in the following critical areas:

(a) development of ultrafast $\mathrm{x}$-ray generation and measurement techniques at $3^{\text {rd }}$ generation synchrotrons

(b) synchrotron beamlines and instrumentation

(c) insertion devices for $3^{\text {rd }}$ generation synchrotrons

(d) laser/electron beam interactions and accelerator physics 
The UXS facility will operate in the spirit of a collaborative research center. Because ultrafast $\mathrm{x}$-ray science is a rapidly emerging field, it is important that scientific applications and experiments be developed in synergy with new instrumentation and measurement techniques. Experiments will be conducted in collaborative groups (of the core research team) comprised of researchers with complementary scientific and technical expertise. The scientific development of the UXS facility will be guided by a Beamline Steering Committee. Committee membership will rotate on an annual basis, and the proposed initial committee structure is as follows:

$\begin{array}{llll}\text { co-chair } & \text { Roger W. Falcone } & \text { Physics Department } & \text { U.C. Berkeley } \\ \text { co-chair } & \text { Robert W. Schoenlein } & \text { Materials Sciences Division } & \text { LBNL } \\ & \text { N. Berrah } & \text { Physics Department } & \text { University of Western Michigan } \\ \text { M. Chergui } & \text { Inst. of Condensed Matter Physics } & \text { University of Lausanne } \\ \text { P.A. Heimann } & \text { Advanced Light Source } & \text { LBNL } \\ \text { C.V. Shank } & \text { Chemistry Department } & \text { U.C. Berkeley } \\ & & \text { Materials Sciences Division } & \text { LBNL }\end{array}$

The primary functions of the Beamline Steering Committee are to guide the scientific development of the UXS facility and to determine experimental priorities among the various collaborative sub-groups of the core research team. Actions of the Beamline Steering Committee are of course subject to the approval of the ALS Scientific Advisory Committee. Beam time for other UXS facility users (outside the core research group) will be allocated according to the existing peer-reviewed Independent Investigator Program at the ALS. For the initial start-up period, we propose to allocate most of the beam time to the core research team and to facility development activities. Following this period, the proposed allocation of beam time is as follows:

Independent Investigators $\quad 30 \%$

Time-Resolved X-ray Research Development $\quad 15 \%$

Core Research Team $55 \%$

This proposed distribution is also subject to regular review and approval by the ALS Scientific Advisory Committee.

The technical design and construction of the UXS facility will be supervised by a project manager selected by the Beamline Steering Committee together with ALS management. Personnel for critical components of this project have already been identified. The development of the superconducting undulator will be headed by Ross Schlueter (LBNL Engineering Division) who leads the engineering team that has developed all the insertion-devices at the ALS. Design of the UXS beamline will be supervised by Howard Padmore (Head - ALS Experimental Systems Group) and Phil Heimann (ALS Experimental Systems Group) who recently supervised the development of the femtosecond bend-magnet beamline (BL 5.3.1) at the ALS. The modifications to the ALS storage-ring lattice (vertical dispersion bump, and mini-beta function) will be supervised by David Robin (Head - ALS Accelerator Physics Group) and by Alexander Zholents (LBNL Center for Beam Physics). Dr. Zholents conceived the original scheme for generating femtosecond x-rays from a storage ring, and has been instrumental in the development of this technique at the ALS. Robert Schoenlein will oversee the development of the femtosecond laser system and the integration of commercial components by LBNL Engineering. 


\section{Appendix - Assessment of Experimental Feasibility}

This appendix provides feasibility assessments and estimates of data acquisition times for the various classes of experiments that are anticipated in the scientific program for the Ultrafast $\mathrm{X}$-ray Science Facility. These estimates also serve to illustrate the demanding requirements that such experiments impose on the x-ray flux and brightness from the beamline. In most examples, a signal-to-noise ratio $(\mathrm{S} / \mathrm{N})$ of 10 is chosen as a convenient benchmark. The required $\mathrm{S} / \mathrm{N}$ depends on the particular experiment and the time-evolution of the signal of interest. Note that for shot-noise-limited measurements, $S / N \sim I_{x-r a y}{ }^{1 / 2}$, where $I_{x-r a y}$ is the integrated $\mathrm{x}$-ray flux (number of x-ray photons).

\section{Order-Disorder Transitions in Solids and at Surfaces}

Feasibility assessments of time resolved Bragg diffraction measurements of laser-induced disorder in crystalline systems are based on previous experimental measurements. For example, measurements illustrated in Figs. 3 and 4 [9] were made using a femtosecond x-ray source based on Thomson scattering [15] which provided an average femtosecond x-ray flux which was a factor of $\sim 10^{-5}$ (brightness factor: $\sim 10^{-9}$ ) of the proposed undulator beamline[29]. Measurements illustrated in Fig. 5 were made using a bend-magnet beamline at the ALS providing flux which was $\sim 10^{-2}$ (brightness factor: $\sim 10^{-3}$ ) of the proposed undulator beamline. In addition, the measurements in Fig. 5 were made at a laser repetition rate of only $1 \mathrm{kHz}$, compared with the $40 \mathrm{kHz}$ system proposed for the undulator beamline. This represents yet another factor of 40 improvement in average flux and brightness for laser-pump, $\mathrm{x}$-ray probe experiments. It is clear that the undulator beamline will enable time-resolved Bragg diffraction measurements with ultrafast time resolution and extremely high signal-to-noise $(\mathrm{S} / \mathrm{N})$ ratios. This is a significant advantage for quantitative interpretation of the underlying dynamics.

For time-resolved EXAFS of order-disorder transitions, the undulator beamline will enable high-sensitivity measurements of ultrafast changes in bond distances and nearest-neighbor coordination. The technical challenge is to achieve sufficient surface sensitivity and signal-tonoise to distinguish small changes in the EXAFS.

For a uniform sample of a single constituent atom, the EXAFS signal-to-noise ratio (measured in transmission) is given by [163]:

$$
S / N=0.7 \frac{\Delta \mu}{\mu} I_{o}^{1 / 2}
$$

where $I_{0}$ is the integrated incident x-ray flux (number of photons), $\mu=N_{s} \cdot \sigma_{s}$ where $N_{s}$ is the sample concentration, $\sigma_{s}$ is the absorption cross-section of the sample in the x-ray region of interest $\left(\Delta \sigma_{s}\right.$ is the expected change in the x-ray cross section). The factor 0.7 results from the optimization of the $\mathrm{S} / \mathrm{N}$ with respect to the sample thickness.

For example, the calculated Si-EXAFS in Fig. 6 illustrates changes in the EXAFS signal of $\sim 10 \%$ of the K-edge absorption. This establishes a minimum requirement of $\sim 200 \mathrm{x}$-ray photons (assuming that the dominant noise contribution is shot noise) to reach $\mathrm{S} / \mathrm{N}=1$, which is 
easily achieved. However, the challenge for these types of experiments is to match the x-ray probing depth to the optical excitation depth. If the photoelectron yield is detected as a means of providing surface sensitivity, then the signal-to-noise ratio can be written as:

$$
S / N=\frac{\Delta \mu}{\mu} I_{o}^{1 / 2} \eta_{c o l}^{1 / 2} \eta_{e}^{1 / 2}\left(d_{e} \mu\right)^{1 / 2}
$$

where $\eta_{\text {col }} \sim 1 \%$ (conservatively) is the photoelectron collection efficiency, $\eta_{\mathrm{e}}$ is the photoelectron yield $\left(\eta_{\mathrm{e}} \sim 1\right.$ for $\left.Z<20\right)$ and $d_{\mathrm{e}} \mu$ is the ratio of the electron escape depth $\left(\mathrm{d}_{\mathrm{e}}\right)$ to the $\mathrm{x}$-ray absorption length $\left(\mu^{-1}\right)$. In the case of $\mathrm{Si}$, at the K-edge, the $\mathrm{x}$-ray penetration depth is $\sim 10^{-6} \mathrm{~m}$ and the photoelectron escape depth is typically $\sim 10^{-8} \mathrm{~m}$. Thus, $\sim 10^{6}$ photons are required to achieve $\mathrm{S} / \mathrm{N} \sim 1$, and this corresponds to $\sim 0.25 \mathrm{msec}$ of integration on the proposed undulator beamline, operating with the full $\mathrm{x}$-ray pulse length, or $\sim 0.4$ seconds of integration using the femtosecond $\mathrm{x}$-ray pulses.

A more effective approach for achieving surface sensitivity for these types of experiments is to measure the EXAFS via reflectivity at grazing incidence angles. For reflectivity measurements, the signal-to-noise ratio is given by:

$$
S / N=\frac{\Delta R}{R}\left(I_{o} R\right)^{1 / 2}\left(d_{o p t} \mu\right)^{1 / 2}
$$

where $\mathrm{R}$ is the reflectivity, $\mathrm{d}_{\mathrm{opt}}$ is the optical excitation depth, and $\mu^{-1}$ is the $\mathrm{x}$-ray penetration depth. In the case of $\mathrm{Si}$, the reflectivity at the $\mathrm{K}$-edge is $\mathrm{R}_{\mathrm{K}}=1.5 \times 10^{-2}$, and the reflectivity change across the K-edge is $\Delta \mathrm{R}_{\mathrm{K}}=1.2 \times 10^{-2}$ at an incident angle of $1^{\circ}$. For the $10 \%$ modulations indicated in Fig. 6 , this gives $\Delta \mathrm{R} / \mathrm{R}=8 \times 10^{-2}$. Furthermore, the optical excitation depth can easily be matched to the $188 \AA \mathrm{x}$-ray penetration depth at this angle. Thus, $\sim 1 \times 10^{4}$ photons are required to achieve $\mathrm{S} / \mathrm{N} \sim 1$, and this corresponds to $\sim 2 \mu \mathrm{sec}$ of integration on the proposed undulator beamline, operating with the full x-ray pulse length, or $\sim 3 \mathrm{msec}$ of integration using the femtosecond $\mathrm{x}$-ray pulses.

\section{Dynamics in Warm Dense Matter}

The objective of these experiments is to study materials at temperatures of a few $\mathrm{eV}$ and near solid density. These conditions exist in laser-heated foils after heat is transported through the depth of the sample and before significant expansion of the surfaces occurs. Hydrodynamic simulations of an $\mathrm{Al}$ foil, $100 \mathrm{~nm}$ thick, show nearly uniform temperature and density $10 \mathrm{ps}$ after the laser pulse. This time scale matches well the time resolution of the streak camera. An important consideration for these experiments is that the sample must be translated between laser shots (since laser heating to high temperature makes a small hole in the foil). Tests using a sample manipulator at beamline 5.3.1 confirm that the foil samples can be translated quickly for $10 \mathrm{~Hz}$ data acquisition. Use of the grating in dispersive mode allows the entire XANES spectrum to be collected at once (the spectrum is dispersed along the streak camera slit). For Al at the $\mathrm{K}$-edge, the foil thickness, $100 \mathrm{~nm}$, is less than the x-ray attenuation length, $900 \mathrm{~nm}$. From this absorption factor, we consider a requirement of $10^{4}$ detected photons / resolution element / $10 \mathrm{ps}$ 
for $\mathrm{S} / \mathrm{N} \sim 100$. The streak camera quantum efficiency at the Al K edge is $\sim 5 \%$. Taking into account these factors implies that these measurements can be made in 14 laser shots or $1.4 \mathrm{sec}$.

Requirements for WDM measurements with the 200 fs x-ray pulses follow from the same considerations with the following corrections. First, the efficiency factor of $7 \times 10^{-4}$ in generating the femtosecond x-rays (see section VI.3 and Fig. 23) results in an increase in the integration time by a factor of $\left(7 \times 10^{-4}\right)^{-1}$. However, the streak camera is then replaced by an integrating detector (CCD or photodiode array) with nearly unity quantum efficiency which reduces the integration time by a factor of 20 (100\% vs. 5\% detector efficiency) and another factor of 3 (since it is no longer necessary to gate $10 \mathrm{ps}$ out of the $30 \mathrm{ps} \mathrm{x}$-ray pulse). This implies that measurements with $\mathrm{S} / \mathrm{N} \sim 100$ can be made with $\sim 330$ shots, or $33 \mathrm{sec}$ integration time at $10 \mathrm{~Hz}$ repetition rate.

\section{Ultrafast Photochemical Reactions}

For studies of structural dynamics of dilute samples (nanocrystals, molecules, atoms) in a solvent environment (or in gas phase) using x-ray absorption spectroscopy (EXAFS or XANES for example), one measures the transmitted $x$-ray intensity, $I_{1}$, given by:

$$
I_{1}=I_{0} e^{-N_{s} F\left(\sigma_{s}+\Delta \sigma_{s}\right) d} e^{-N_{s}(1-F) \sigma_{s} d} e^{-\alpha_{s o l v} d}
$$

where $I_{0}$ is the incident x-ray flux, $N_{s}$ is the sample concentration, $\sigma_{s}$ is the absorption crosssection of the sample in the $\mathrm{x}$-ray region of interest $\left(\Delta \sigma_{s}\right.$ is the expected change in the x-ray cross section due to transient structural changes induced by a laser excitation pulse), $d$ is the sample thickness, and $\alpha_{\text {solv }}$ is the attenuation length of the solvent (sample environment). $F$ is the photolysis factor (i.e. the fraction of the samples that are excited by the laser pump pulse) and is given by:

$$
F=\frac{\Phi \sigma_{o p t}}{d} \int_{0}^{d} J_{o p t}(z) d z=\frac{J_{o p t}(0) \Phi}{N_{s} d}\left(1-e^{-N_{s} \sigma_{o p t} d}\right) \quad 0<F<1
$$

where $\sigma_{\text {opt }}$ is the optical absorption cross-section, $\Phi$ is the quantum efficiency of the photoinduced process, and $\mathrm{J}_{\mathrm{opt}}$ is the incident laser photon fluence.

The signal of interest is the differential of the x-ray transmission (with vs. without the optical pump pulse), which in the small-signal limit is given by:

$$
S=I_{1} N_{s} F \Delta \sigma_{s} d \quad \text { for } \quad N_{s} F \Delta \sigma_{s} d<<1
$$

The shot noise is $\mathrm{N}=\mathrm{I}_{1}{ }^{1 / 2}$, thus the signal-to-noise ratio is given by:

$$
S / N=J_{o p t}(0) \Phi\left(1-e^{-N_{s} \sigma_{\text {opt }} d}\right) I_{1}^{1 / 2} e^{-N_{s} \sigma_{s} d / 2} e^{-\alpha_{s o l v} d / 2} \Delta \sigma_{s}
$$


The signal-to-noise can thus be optimized for particular experiments in terms of the sample concentration, the X-ray absorption cross-section (by choice of K-edge or L-edge for example), the optical absorption cross section (via judicious choice of the excitation wavelength), and the sample thickness. Note that the solvent (or sample environment) absorption always degrades the signal-to-noise ratio and does not influence the optimum choice of sample concentration or cross section. Furthermore, enhancement of the signal via increases in $\mathrm{J}_{\text {opt }}$ may be limited in some cases by non-linear optical effects in the solvent, and/or by undesired multiphoton effects in the sample (at photon densities on the order of or greater than one per optical absorption cross-section). For many systems which have small two-photon absorption cross-sections, multiphoton effects in the sample will not be an issue. In addition, it is generally possible to excite samples slightly off-resonance (where $\sigma_{\text {opt }}$ is reduced) thereby reducing the probability of multiphoton absorption while maintaining the overall photolysis factor.

Figure A1 illustrates the dependence of $\mathrm{S} / \mathrm{N}$ on the relative optical and $\mathrm{x}$-ray absorption cross sections and the sample concentration. Note that for $\sigma_{\text {opt }}>>\sigma_{\mathrm{s}}$ (as is typically the case) the $\mathrm{S} / \mathrm{N}$ reaches a maximum at relatively low sample concentrations (at which the sample is optically opaque but largely transparent at x-ray wavelengths). Under these conditions, all of the optical photons are absorbed in the sample and the unexcited molecules simply degrade the achievable $\mathrm{S} / \mathrm{N}$ by attenuating the transmitted $\mathrm{x}$-rays. Similarly, increasing the sample concentration or sample thickness diminishes the $\mathrm{S} / \mathrm{N}$. For $\sigma_{\mathrm{opt}}>\sigma_{\mathrm{s}}$, reducing the optical absorption cross section (for example by exciting off-resonance) to match the x-ray cross-section does not improve the $\mathrm{S} / \mathrm{N}$. The $\mathrm{S} / \mathrm{N}$ can be improved by probing at edges where differential absorption changes, $\Delta \sigma_{\mathrm{s}}$, are larger (L-edge vs. K-edge for example), provided that these benefits outweigh the degradation of the $\mathrm{S} / \mathrm{N}$ due to stronger overall sample absorption, $\sigma_{\mathrm{s}}$, and solvent absorption, $1 / \alpha_{\text {solv }}$.

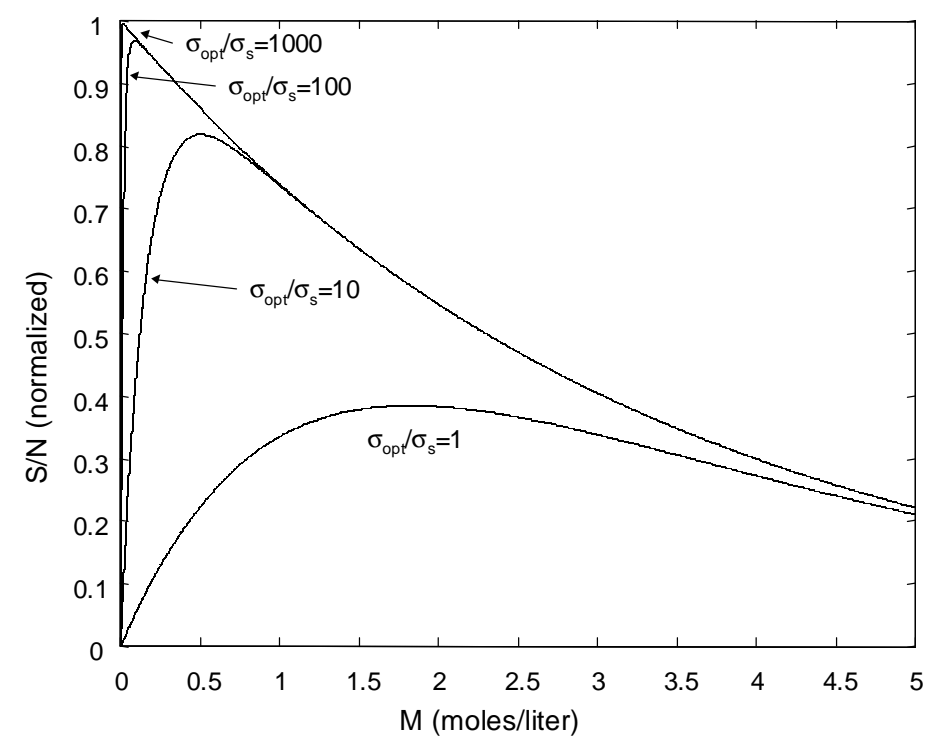

Figure A1. Dependence of $\mathrm{S} / \mathrm{N}$ (calculated from Eq. A7, normalized to $\mathrm{J}_{\mathrm{o}} \cdot \Phi \cdot \mathrm{I}_{1}{ }^{1 / 2} \cdot \Delta \sigma_{\mathrm{s}}$ ) on the relative optical and $\mathrm{x}$-ray absorption cross sections and the sample concentration with $\sigma_{\mathrm{s}}=10^{-19} \mathrm{~cm}^{2}, \mathrm{~d}=.01 \mathrm{~cm}$, and $\alpha_{\text {solv }}=0 \mathrm{~cm}^{-1}$. 
Table A1 provides a comparison of the source requirements to measure a core-level absorption edge jump at $\mathrm{S} / \mathrm{N}=10$ for various $\mathrm{x}$-ray absorption experiments at the K-edge or L-edge of the solute molecule. The chosen parameters and experimental conditions represent a rough optimization of the $\mathrm{S} / \mathrm{N}$ based on Eq. A7. The change in the x-ray absorption crosssection, $\Delta \sigma_{\mathrm{x} \text {-ray }}$, refers to the absorption change at the corresponding core level $(\mathrm{K}$ or $\mathrm{L})$ for that molecule (and is taken from tabulated values). In some cases, the optical excitation is detuned from the absorption maximum in order to reduce the absorption cross-section, $\sigma_{\mathrm{opt}}$, and thereby avoid mulitphoton effects. This is conservative, and may not be necessary since the excited-state $\left(\mathrm{S}_{1}-\mathrm{S}_{\mathrm{n}}\right)$ absorption cross-section for most molecules is much smaller that the ground-state absorption cross-section $\left(\mathrm{S}_{0}-\mathrm{S}_{1}\right)$. The assumed optical fluence, $\mathrm{J}_{\mathrm{opt}}$, is chosen to limit the peak optical intensity to $10^{11}-10^{12} \mathrm{~W} / \mathrm{cm}^{2}$ in order to avoid nonlinear effects in the solvent. In practice, substantially higher intensities may be tolerated since very thin samples are used $(\mathrm{d} \sim 100 \mu \mathrm{m})$. Finally, the integration time (to achieve a benchmark $\mathrm{S} / \mathrm{N}=10$ ) is indicated for each experiment. Integration times are determined directly from the flux curves in Fig. 23 for measurements using the full synchrotron pulse duration ('psec' column). Integration times for experiments using the $200 \mathrm{fs}$ x-rays ('fsec' column) are determined from the flux curves in Fig. 23 normalized by the factor, $7 \times 10^{-4}$, which accounts for the slicing efficiency (see Figs. 18 and 23 and section VI.3).

The signal of interest depends on the particular experiment and the anticipated time dynamics. The benchmark used in Table A1 of $\mathrm{S} / \mathrm{N}=10$ (for which the signal is defined as a $\mathrm{K}$ or $\mathrm{L}$ absorption edge jump) is adequate for discerning the dynamics of a significant edge shift or a near edge resonance feature (see Fig. 14 for example). Measurements of dynamic features in the EXAFS will likely require $\mathrm{S} / \mathrm{N}>100$ (since the expected signals are typically several percent of the absorption edge jump - see EXAFS calculations in Figs. 6, 10, and 13 for example). For such experiments, the indicated integration times should be scaled by at least x 100 . These estimates provide only a baseline, and are based on the simplifying assumptions of (a) shot-noise limited detection, and (b) $100 \%$ detection efficiency. Additional integration time will likely be required for non-ideal detection systems. Furthermore, collecting multiple energy points (scanning spectra) and multiple time points to map dynamics will result in corresponding increases in integration time. These effects may be mitigated to some extent by the extension of dispersive EXAFS techniques to $10 \mathrm{keV}$. 


\begin{tabular}{|c|c|c|c|c|c|c|}
\hline System & \multicolumn{2}{|c|}{$\mathrm{CdSe}$ in $\mathrm{C}_{8} \mathrm{H}_{16}$} & \multicolumn{2}{|c|}{$[\mathrm{Fe}($ tpen $)]\left(\mathrm{ClO}_{4}\right)_{2}$ in $\mathrm{H}_{2} \mathrm{O}$} & \multicolumn{2}{|c|}{$\mathrm{I}_{2}$ in $n$-hexane } \\
\hline M (moles/liter) & \multicolumn{2}{|c|}{0.1} & \multicolumn{2}{|c|}{0.26} & \multicolumn{2}{|c|}{0.27} \\
\hline X-ray absorption edge & \multicolumn{2}{|c|}{$\mathrm{Cd}\left(\mathrm{L}_{\text {edge }}\right) 3.54 \mathrm{keV}$} & \multicolumn{2}{|c|}{$\mathrm{Fe}\left(\mathrm{K}_{\text {edge }}\right) 7.11 \mathrm{keV}$} & \multicolumn{2}{|c|}{$\mathrm{I}\left(\mathrm{L}_{\text {edge }}\right) 5.19 \mathrm{keV}$} \\
\hline Signal: $\Delta \sigma_{\mathrm{x} \text {-ray }}\left(\times 10^{-19} \mathrm{~cm}^{2}\right)$ & \multicolumn{2}{|c|}{1.6} & \multicolumn{2}{|c|}{0.33} & \multicolumn{2}{|c|}{0.26} \\
\hline $\mathrm{d}(\mu \mathrm{m})$ & \multicolumn{2}{|c|}{100} & \multicolumn{2}{|c|}{100} & \multicolumn{2}{|c|}{100} \\
\hline$\sigma_{\mathrm{opt}}\left(\times 10^{-17} \mathrm{~cm}^{2}\right)$ & \multicolumn{2}{|c|}{$20($ at $h v \approx 2.5 \mathrm{eV})$} & \multicolumn{2}{|c|}{$0.12($ at $h v \approx 2.7 \mathrm{eV})$} & \multicolumn{2}{|c|}{$.1($ at $v \approx 2.3 \mathrm{eV})$} \\
\hline $\mathrm{J}_{\mathrm{opt}}\left(\times 10^{17} \mathrm{ph} / \mathrm{cm}^{2}\right)$ & \multicolumn{2}{|c|}{0.5} & \multicolumn{2}{|c|}{4.3} & \multicolumn{2}{|c|}{5.2} \\
\hline $\mathrm{X}$-ray ph req. $(\mathrm{S} / \mathrm{N}=10)$ & \multicolumn{2}{|c|}{$2.8 \times 10^{6}$} & \multicolumn{2}{|c|}{$8.7 \times 10^{5}$} & \multicolumn{2}{|c|}{$1.3 \times 10^{6}$} \\
\hline Time resolution & psec & fsec & psec & fsec & Psec & fsec \\
\hline Integration time $(\mathrm{S} / \mathrm{N}=10)$ & $1 \mathrm{msec}$ & $1.4 \mathrm{sec}$ & $0.2 \mathrm{msec}$ & $0.2 \mathrm{sec}$ & $0.3 \mathrm{msec}$ & $0.4 \mathrm{sec}$ \\
\hline
\end{tabular}

\begin{tabular}{|c|c|c|c|c|c|c|}
\hline System & \multicolumn{2}{|c|}{$\mathrm{ClO}_{2}$ in $\mathrm{H}_{2} \mathrm{O}$} & \multicolumn{2}{|c|}{$\mathrm{NaI}$ in $\mathrm{H}_{2} \mathrm{O}$} & \multicolumn{2}{|c|}{$\mathrm{Hb}-\mathrm{H}_{2} \mathrm{~S}$ in $\mathrm{H}_{2} \mathrm{O}$} \\
\hline M (moles/liter) & \multicolumn{2}{|c|}{0.25} & \multicolumn{2}{|c|}{0.27} & \multicolumn{2}{|c|}{0.005} \\
\hline X-ray absorption edge & \multicolumn{2}{|c|}{$\mathrm{Cl}\left(\mathrm{K}_{\text {edge }}\right) 2.83 \mathrm{keV}$} & \multicolumn{2}{|c|}{$\mathrm{I}\left(\mathrm{L}_{\text {edge }}\right) 5.19 \mathrm{keV}$} & \multicolumn{2}{|c|}{$\mathrm{Fe}\left(\mathrm{K}_{\text {edge }}\right) 7.11 \mathrm{keV}$} \\
\hline Signal: $\Delta \sigma_{\mathrm{x} \text {-ray }}\left(\times 10^{-19} \mathrm{~cm}^{2}\right)$ & \multicolumn{2}{|c|}{0.92} & \multicolumn{2}{|c|}{0.26} & \multicolumn{2}{|c|}{0.46} \\
\hline $\mathrm{d}(\mu \mathrm{m})$ & \multicolumn{2}{|c|}{100} & \multicolumn{2}{|c|}{100} & \multicolumn{2}{|c|}{100} \\
\hline$\sigma_{\text {opt }}\left(\times 10^{-17} \mathrm{~cm}^{2}\right)$ & \multicolumn{2}{|c|}{$0.34($ at $h v \approx 3.1 \mathrm{eV})$} & \multicolumn{2}{|c|}{$0.2($ at $h v \approx 4.9 \mathrm{eV})$} & \multicolumn{2}{|c|}{$0.2($ at $h v \approx 4.9 \mathrm{eV})$} \\
\hline $\mathrm{J}_{\mathrm{opt}}\left(\times 10^{17} \mathrm{ph} / \mathrm{cm}^{2}\right)$ & \multicolumn{2}{|c|}{4.0} & \multicolumn{2}{|c|}{2.6} & \multicolumn{2}{|c|}{1} \\
\hline $\mathrm{X}$-ray ph req. $(\mathrm{S} / \mathrm{N}=10)$ & \multicolumn{2}{|c|}{$1.2 \times 10^{6}$} & \multicolumn{2}{|c|}{$3.0 \times 10^{7}$} & \multicolumn{2}{|c|}{$5 \times 10^{10}$} \\
\hline Time resolution & psec & fsec & psec & fsec & psec & fsec \\
\hline Integration time $(\mathrm{S} / \mathrm{N}=10)$ & $0.3 \mathrm{msec}$ & $0.5 \mathrm{sec}$ & $6 \mathrm{msec}$ & $9 \mathrm{sec}$ & $9 \mathrm{sec}$ & $3.5 \mathrm{hrs}$. \\
\hline
\end{tabular}

Table A1. Feasibility estimates for various x-ray absorption experiments. Estimates provide only a baseline, and are based on the simplifying assumptions of (a) shot-noise limited detection, and (b) $100 \%$ detection efficiency. Additional integration time will likely be required for non-ideal detection systems. Collecting multiple energy points (scanning spectra) and multiple time points to map dynamics will result in corresponding increases in integration time. For the $\mathrm{Hb}_{-} \mathrm{H}_{2} \mathrm{~S}$ system, estimates are based on fluorescence measurements with a $10 \%$ collection efficiency and a $33 \%$ fluorescence quantum yield for Fe.

\section{Atomic and Molecular Physics with Femtosecond Optical and X-ray Pulses}

\section{Low-Field Regime}

For AMO experiments, the time necessary to record a typical photoelectron spectrum (in a femtosecond laser pump and x-ray probe experiment) is estimated based on the known integration times for measuring photoelectron spectra at particular x-ray flux levels. For example, a photoelectron spectrum of $\mathrm{CO}$ (in the energy region of the oxygen $\mathrm{K}$ edge) under typical source pressures, can be recorded in 40 minutes with a signal to noise ratio of $\sim 10^{5}$. For these measurements, the flux is $\sim 10^{11}$ photons/sec of monochromatized light. In a pump-probe experiment, we assume that we can excite $2 \%$ of the sample with the pump laser, resulting in an increase by a factor of 50 in the counting time. From Fig. 23, (scaled by $7 \times 10^{-4}$, see 
section VI.3), the flux of the monochromatized, femtosecond $\mathrm{x}$-ray pulse is $\sim 5 \times 10^{6} \mathrm{photons} / \mathrm{sec}$. In this case, the probe signal will be linear in the incident flux, resulting in an increase in counting time by a factor of $2 \times 10^{4}$. If we assume the pump-probe experiments will be done at the same x-ray energies, the cross sections of interest will be the same as in the probe only spectra. (It should be noted, however, that at higher energies the $\mathrm{x}$-ray absorption cross sections are reduced, which will add another factor into this estimate of counting times.) Considering these factors, it should be possible to achieve a S/N ratio of 100 with an integration time of 40 minutes (assuming that counting statistics are the dominant contribution to the noise). The S/N can be further improved (or the integration time reduced) if we are able to excite a larger fraction of the sample. In general, it is not unreasonable to expect to be able to excite $\sim 10 \%$ of the sample.

\section{High-Field Regime}

We estimate data acquisition times for the high-field, AMO experiments by considering two classes of experiments: (1) experiments on laser-induced modifications to x-ray photoelectron spectra and (2) experiments on x-ray/laser two-color absorption spectra.

Data acquisition times are estimated for a signal-to-noise $(\mathrm{S} / \mathrm{N})$ ratio of 10 and we use the fact that if signal events are detected in the absence of a background, then the number of signal photons one must collect is given by $(\mathrm{S} / \mathrm{N})^{2}$ (i.e. 100 signal photons are required to achieve a $\mathrm{S} / \mathrm{N}=10$ ). If, however, the signal sits on top of a background which is R-times larger than the signal, then the number of collected signal photons required to achieve a given $\mathrm{S} / \mathrm{N}$ is: $(\mathrm{S} / \mathrm{N})^{2}(1+\mathrm{R})$. Additionally, we assume gas phase interactions at an atom-density of $10^{18} \mathrm{~cm}^{-3}$ (25 Torr pressure); this is consistent with operation of a pulsed-gas-valve at a $1 \mathrm{kHz}$ repetition rate. Given the $1 \mathrm{kHz}$ valve repetition rate, we assume a typical beamline flux of $\sim 10^{5} \mathrm{ph} / \mathrm{s}$ (nominal x-ray flux for femtosecond pulses at a few $\mathrm{keV}$, reduced by the ratio of gas-valve to laser repetition rates, 1/40, see Fig. 23 and section VI.3).

With respect to the observation of optical scattering sidebands in $\mathrm{x}$-ray photoelectron spectra, we estimate the time required to observe first order optical scattering. The probability for an x-ray photoelectron to scatter one laser photon [127] can be approximated as :

$$
P_{l}=\left(13.6 \times 10^{-14}\right) \cdot \lambda_{l}^{4} \cdot I_{\text {laser }} \cdot E_{\text {free }}
$$

Here the laser wavelength, $\lambda_{l}$, is in microns, the laser intensity, $\mathrm{I}_{\text {laser }}$, is in $\mathrm{W} / \mathrm{cm}^{2}$ and the laser-field-free photoelectron energy, $\mathrm{E}_{\text {free }}$, is in $\mathrm{eV}$. In writing Eq. A8 we have assumed that the scattering probability is not too high $(\leqslant 25 \%)$. Eq. A8 indicates that scattering probabilities of $\sim 10 \%$ are easily achievable $\left(\mathrm{E}_{\text {free }}=100 \mathrm{eV}, \lambda_{l}=1 \mu \mathrm{m}, \mathrm{I}_{\text {laser }}=10^{10} \mathrm{~W} / \mathrm{cm}^{2}\right.$ for instance). Given $\mathrm{N}_{\mathrm{o}}$ $\mathrm{X}$-ray photons incident on a gas sample, the number of detected electrons, $\mathrm{N}_{\mathrm{e}}$, with energy corresponding to first-order optical scattering is given by:

$$
N_{l}=N_{o} \cdot\left(0.1 \cdot \sigma_{x}\right) \cdot \eta_{\text {det }} \cdot \rho_{\text {gas }} \cdot \ell
$$

Here $\rho_{\text {gas }}$ is the gas density, $\sigma_{\mathrm{x}}$ is the x-ray photoionization cross section, $\ell$ is the interaction length, $\eta_{\text {det }}$ is the detection efficiency (electron collection efficiency and quantum 
efficiency of electron detector), and we have assumed a $10 \%$ optical scattering probability. We next assume a $1 \%$ detection efficiency (10\% collection efficiency and $10 \%$ quantum efficiency), an X-ray photoabsorption cross-section of $10^{-19} \mathrm{~cm}^{2}$, a $1 \mathrm{~mm}$ interaction length, and a gas density of $10^{18} \mathrm{~cm}^{-3}$. One therefore detects $\left(10^{-5}\right)\left(\mathrm{N}_{0}\right)$ electrons. For background-free detection, a S/N ratio of 10 is obtained with 100 detected electrons or equivalently $10^{7}$ incident $\mathrm{X}$-ray photons. At a beamline flux of $10^{5} \mathrm{ph} / \mathrm{s}$ this corresponds to an acquisition time of 100 seconds. While detection of optical scattering peaks should be background free, the data acquisition time will increase as indicated above if these peaks sit on top of a background (due, for instance, to noisecounts or a spectrally wide unperturbed x-ray peak).

Data acquisition times for a two-color photoabsorption process can be estimated by writing an expression analogous to Eq. A9:

$$
N_{d}=N_{0} \cdot\left(f \cdot \sigma_{x}\right) \eta_{A} \cdot \eta_{d e t} \cdot \rho_{g a s} \cdot \ell
$$

Here $\mathrm{N}_{\mathrm{d}}$ is the number of detected particles (photons for transmission experiments or Auger electrons/photons for 'background-free' experiments), $\eta_{\mathrm{A}}$ is an efficiency factor which represents the Auger yield for 'background-free' experiments and is unity for transmission experiments, $f \cdot \sigma_{\mathrm{x}}$ is the cross-section for two photon absorption, and the remaining quantities are defined above (for optical scattering experiments). The two-photon cross-section can be estimated by writing the expression (derived in perturbation theory [164]) for a two-photon transition between an initial state, $i$, through an intermediate state, $k$, to a final state, $f$ :

$$
W_{\text {two-photon }}=\left[\frac{d_{f k} E_{L}}{2\left(\hbar \omega_{k i}-\hbar \omega_{x}\right)}\right]^{2}\left[\frac{2}{\hbar} \frac{\left(d_{k i} E_{x}\right)^{2}}{\Gamma}\right]
$$

Here $E_{x}$ and $E_{L}$ are the $x$-ray and laser field strengths, $d_{a b}$ is the dipole matrix element between states a and b, $\Gamma$ is the energy width of the upper states, $\hbar \omega_{\mathrm{x}}$ is the $\mathrm{x}$-ray photon energy, and $\hbar \omega_{k i}$ is the energy difference between initial and intermediate states. The first bracketed term in Eq. A11 represents the laser dipole transition while the second term represents the $\mathrm{x}$-ray transition. Inspection of Eq. A11 indicates that one can write the two-photon cross-section as a product, $f \cdot \sigma_{\mathrm{x}}$, of a nominal $\mathrm{x}$-ray absorption cross-section, $\sigma_{\mathrm{x}}$, and a dimensionless factor, $f=$ $\left[\left(\mathrm{d}_{f k} \cdot \mathrm{E}_{\mathrm{L}}\right) / 2\left(\hbar \omega_{k i}-\mathrm{h} \omega_{\mathrm{x}}\right)\right]^{2}$. We estimate this factor, $f$, for a $1 \mathrm{~s} \rightarrow 3 \mathrm{~d} \rightarrow 4 \mathrm{p}$ transition in Ar to be $f=$ $5 \times 10^{-15} \mathrm{I}_{\text {laser }}$ where the laser intensity is in $\mathrm{W} / \mathrm{cm}^{2}$. With a nominal Ar K-edge absorption cross section of $\sim 10^{-19} \mathrm{~cm}^{2}$, the two-photon cross section is $\sim 5 \times 10^{-21} \mathrm{~cm}^{2}$ for a laser intensity of $10^{13} \mathrm{~W} / \mathrm{cm}^{2}$.

Photoabsorption experiments will typically be performed in a 'background-free' configuration where one detects Auger electrons or photons as a signature of a two-color-driven core-shell hole. Assuming an auger yield $\left(\eta_{\mathrm{A}}\right)$ of $10 \%$, a two-photon cross-section of $5 \times 10^{-21} \mathrm{~cm}^{2}$, a detection efficiency of $1 \%$, an interaction length of $1 \mathrm{~mm}$, and a gas density of $10^{18} \mathrm{~cm}^{-3}$, one detects $\left(5 \times 10^{-7}\right)\left(\mathrm{N}_{\mathrm{o}}\right)$ particles. At a beamline flux of $10^{5} \mathrm{ph} / \mathrm{s}$, a S/N ratio of 10 is obtained in 33 minutes $\left(2 \times 10^{8}\right.$ incident photons). 


\section{References}

1. A. Dienes, E. P. Ippen, and C. V. Shank, "A mode-locked cw dye laser," Appl. Phys. Lett., 19, 258-260, 1971.

2. T. Elsaesser, J. G. Fujimoto, D. A. Wiersma, and W. Zinth, "Ultrafast Phenomena XI," in Springer-Verlag Series in Chemical Physics, vol. 63. Berlin: Springer-Verlag, 1998.

3. A. M. Lindenberg, I. Kang, S. L. Johnson, T. Missalla, P. A. Heimann, Z. Chang, J. Larsson, P. H. Bucksbaum, H. C. Kapteyn, H. A. Padmore, R. W. Lee, J. S. Wark, and R. W. Falcone, "Time-resolved x-ray diffraction from coherent phonons during laser-induced phase transition," Phys. Rev. Lett., 84, 111-114, 2000.

4. R. W. Schoenlein, S. Chattopadhyay, H. H. W. Chong, T. E. Glover, P. A. Heimann, C. V. Shank, A. Zholents, and M. Zolotorev, "Generation of femtosecond pulses of synchrotron radiation," Science, 287, 2237-2240, 2000.

5. A. Cavalleri, C. Siders, F. Brown, D. Leitner, C. Toth, J. Squier, B. CPJ, K. Wilson, K. Sokolowski-Tinten, D. von der Linde, M. Horn von Hoegen, and M. Kammler, "Anharmonic lattice dynamics in germanium measured with ultrafast x-ray diffraction," Phys. Rev. Lett., 85, 586-589, 2000.

6. F. L. H. Brown, K. R. Wilson, and J. Cao, "Ultrafast extended x-ray absorption fine structure (EXAFS) - theoretical considerations," J. Chem. Phys., 111, 6238-6246, 1999.

7. I. V. Tomov, D. A. Oulianov, P. Chen, and P. M. Rentzepis, "Ultrafast time-resolved transient structures of solids and liquids studied by means of X-ray diffraction and EXAFS," J. Phys. Chem. B, 103, 7081 -7091, 1999.

8. P. L. Chen, I. V. Tomov, and P. M. Rentzepis, "Lattice dynamics of laser-heated GaAs crystals by means of time-resolved X-ray diffraction," J. Phys. Chem. A, 103, 2359-2363, 1999.

9. A. H. Chin, R. W. Schoenlein, T. E. Glover, P. Balling, W. P. Leemans, and C. V. Shank, "Ultrafast structural dynamics in InSb probed by time-resolved x-ray diffraction," Phys. Rev. Lett., 83, 336-339, 1999.

10. C. W. Siders, A. Cavalleri, K. Sokolowski-Tinten, C. Toth, T. Guo, M. Kammler, M. Horn von Hoegen, K. R. Wilson, D. von der Linde, and C. P. J. Barty, "Detection of nonthermal melting by ultrafast X-ray diffraction," Science, 286, 1340-1342., 1999.

11. C. Rose-Petruck, R. Jimenez, T. Guo, A. Cavalleri, C. Siders, F. Raksi, J. A. Squier, B. C. Walker, K. R. Wilson, and C. P. J. Barty, "Picosecond-milliangstrom lattice dynamics measured by ultrafast X-ray diffraction," Nature, 398, 310-312, 1999.

12. B. Perman, V. Srajer, Z. Ren, T.-Y. Teng, C. Pradervand, T. Ursby, D. Bourgeois, F. Schotte, M. Wulff, R. Kort, K. Hellingwerf, and K. Moffat, "Energy transduction on the nanosecond time scale: Early structural events in a Xanthopsin photocycle," Science, 279, 1946-1950, 1998.

13. J. Larsson, Z. Chang, E. Judd, P. J. Schuck, R. W. Falcone, P. A. Heimann, H. A. Padmore, H. C. Kapteyn, P. H. Bucksbaum, M. M. Murnane, R. W. Lee, A. Machacek, J. S. Wark, X. Liu, and B. Shan, "Ultrafast X-ray diffraction using a streak-camera detector in averaging mode," Opt. Lett., 22, 1012, 1997.

14. M. Wulff, F. Schotte, G.Naylor, D. Bourgeois, K. Moffat, and G. Mourou, "Time-resolved structures of macromolecules at the ESRF: Single pulse Laue diffraction, stroboscopic data 
collection and femtosecond flash photolysis," Nuc. Inst. and Meth. in Phys. Res. A, 398, 69-84, 1997.

15. R. W. Schoenlein, W. P. Leemans, A. H. Chin, P. Volfbeyn, T. E. Glover, P. Balling, M. Zolotorev, K.-J. Kim, S. Chattopadhyay, and C. V. Shank, "Femtosecond x-ray pulses at 0.4 angstroms generated by $90^{\circ}$ Thomson scattering: A tool for probing the structural dynamics of materials," Science, 274, 236-238, 1996.

16. J. Larsson, P. A. Heimann, A. M. Lindenberg, P. J. Schuck, P. H. Bucksbaum, R. W. Lee, H. A. Padmore, J. S. Wark, and R. W. Falcone, "Ultrafast structural changes measured by time-resolved x-ray diffraction," Applied Physics A, 66, 587-591, 1998.

17. T. Anderson, I. V. Tomov, and P. M. Rentzepis, "Laser-driven metal photocathodes for picosecond electron and x-ray pulse generation,” J. Appl. Phys., 71, 5161-5167, 1992.

18. M. M. Murnane, H. C. Kapteyn, M. D. Rosen, and R. W. Falcone, "Ultrafast pulses from laser-produced plasmas," Science, 251, 531-536, 1991.

19. J. P. Bergsma, M. H. Coladonado, P. M. Edelsten, J. D. Hahn, and K. R. Wilson, "Transient x-ray scattering calculated from molecular dynamics," J. Chem. Phys., 84, 6151-6159, 1986.

20. J. R. Buschert, J. Z. Tischler, D. M. Mills, Q. Zhao, and R. Colella, "Time resolved x-ray diffraction study of laser annealing in silicon at grazing incidence," J. Appl. Phys., 66, 3523-3525, 1989.

21. D. M. Mills, A. Lewis, A. Hartoonian, J. Huang, and B. Smith, "Time-resolved X-ray absorption spectroscopy of CO-myoglobin recombination after laser photolysis," Science, 223, 811-813, 1984.

22. M. H. Koch, N. A. Dencher, D. Oesterhelt, H.-J. Plohn, G. Rapp, and G. Buldt, "Timeresolved x-ray diffraction study of the structural changes associated with the photocycle of bacteriorhodopsin,” EMBO Journal, 10, 521-526, 1991.

23. A. L'Huiller, M. Lewenstein, P. Salieres, and P. Balcou, "High-order harmonic-generation cutoff," Phys. Rev. A, 48, R3433-R3436, 1993.

24. Z. Chang, A. Rundquist, H. Wang, M. M. Murnane, and H. C. Kapteyn, "Generation of coherent soft X rays at $2.7 \mathrm{~nm}$ using high harmonics," Phys. Rev. Lett., 79, 2967-2970, 1997.

25. M. Schnurer, C. Spielmann, P. Wobrauschek, C. Streli, N. H. Burnett, C. Kan, K. Ferencz, R. Koppitsch, Z. Cheng, T. Brabec, and F. Krausz, "Coherent 0.5-keV X-ray emission from helium driven by a sub-10-fs laser," Phys. Rev. Lett., 80, 3236-3239., 1998.

26. C. G. Durfee III, A. R. Rundquist, S. Backus, C. Herne, M. M. Murnane, and K. C. Kapteyn, "Phase matching of high-order harmonics in hollow waveguides," Phys. Rev. Lett., 83, 2187-2190, 1999.

27. C. Rischel, A. Rousse, I. Uschmann, P.-A. Albouy, J.-P. Geindre, P. Audebert, J.-C. Gauthier, E. F:orster, J.-L. Martin, and A. Antonetti, "Femtosecond time-resolved X-ray diffraction from laser-heated organic films," Nature, 390, 490-492, 1997.

28. The full report from this panel is available at the following web address: http://www.er.doe.gov/production/bes/BESAC/charges.html

29. R. W. Schoenlein, S. Chattopadhyay, H. H. W. Chong, T. E. Glover, P. A. Heimann, C. V. Shank, A. Zholents, and M. Zolotorev, "Generation of x-ray pulses via laser-electron beam interaction," Appl. Phys. B, 71, 1-10, 2000.

30. A. A. Zholents and M. S. Zolotorev, "Femtosecond x-ray pulses of synchrotron radiation," Phys. Rev. Lett., 76, 912-915, 1996. 
31. K.-J. Kim, S. Chattopadhyay, and C. V. Shank, "Generation of femtosecond x-ray pulses by 90 degree Thomson scattering," Nuc. Inst. and Meth. in Phys. Res. A, 341, 351-354, 1994.

32. B. C. Larson, C. W. White, T. S. Noggle, and J. F. Barhorst, "Time-resolved X-ray diffraction measurement of the temperature and temperature gradients in silicon during pulsed laser annealing," Appl. Phys. Lett., 42, 282-284, 1983.

33. J. A. V. Vechten, R. Tsu, F. W. Saris, and D. Hoonhout, "Reasons to believe pulsed laser annealing of Si does not involve simple thermal melting," Physics Letters, 74A, 417-421, 1979.

34. J. A. V. Vechten, R. Tsu, and F. W. Saris, "Nonthermal pulsed laser annealing of Si; Plasma annealing," Physics Letters, 74A, 422-426, 1979.

35. P. Stampfli and K. H. Bennemann, "Dynamical theory of the laser-induced instability of silicon," Phys. Rev. B, 46, 10686-10692, 1992.

36. P. Stampfli and K. H. Bennemann, "Time dependence of the laser-induced femtosecond lattice instability of Si and GaAs - role of longitudinal optical phonons," Phys. Rev. B, 49, 7299-7305, 1994.

37. P. Stampfli and K. H. Bennemann, "Theory for the laser-induced femtosecond phase transition of silicon and GaAs," Appl. Phys. A, 60, 191-196, 1995.

38. P. L. Silvestrelli, A. Alavi, M. Parrinello, and D. Frenkel, “Ab initio molecular dynamics simulation of laser melting of silicon," Phys. Rev. Lett., 77, 3149-3152, 1996.

39. P. L. Silvestrelli, A. Alavi, M. Parrinello, and D. Frenkel, "Structural, dynamical, electronic, and bonding properties of laser-heated silicon: An ab initio molecular-dynamics study," Phys. Rev. B, 56, 3806-3812, 1997.

40. H. W. K. Tom, T. F. Heinz, and Y. R. Shen, "Second-harmonic reflection from silicon surface and its relation to structural symmetry," Phys. Rev. Lett., 51, 1983-1986, 1983.

41. C. V. Shank, R. Yen, and C. Hirlimann, "Time-resolved reflectivity measurement on femtosecond-optical-pulse-induced phase transitions on silicon," Phys. Rev. Lett., 50, 454457, 1983.

42. C. V. Shank, R. Yen, and C. Hirlimann, "Time-resolved surface structural dynamics of optically excited silicon," Phys. Rev. Lett., 51, 900-902, 1983.

43. M. C. Downer, R. L. Fork, and C. V. Shank, "Femtosecond imaging of melting and evaporation at a photoexcited silicon surface," J. Opt. Soc. Am. B, 2, 595-599, 1985.

44. H. W. K. Tom, G. D. Aumiller, and C. H. Brito-Cruz, "Time-resolved study of laserinduced disorder of Si surfaces," Phys. Rev. Lett., 60, 1438-1441, 1988.

45. Y. Siegal, E. N. Glezer, and E. Mazur, "Dielectric constant of GaAs during a subpicosecond laser-induced phase transition," Phys. Rev. B, 49, 16403-16406, 1994.

46. I. L. Shumay and U. Hofer, "Phase transformation of an InSb surface induced by strong femtosecond laser pulses," Phys. Rev. B, 53, 15878-15884, 1996.

47. K. Sokolowski-Tinten, H. Schulz, J. Bialkowski, and D. von der Linde, "Two distinct transitions in ultrafast solid-liquid phase transformations of GaAs," Appl. Phys. A, 53, 227234, 1991.

48. W. P. Leemans, R. W. Schoenlein, P. Volfbeyn, A. H. Chin, T. E. Glover, P. Balling, M. Zolotorev, K.-J. Kim, S. Chattopadhyay, and C. V. Shank, "X-ray based time resolved electron beam characterization via $90^{\circ}$ Thomson scattering," Phys. Rev. Lett., 77, 41824185, 1996. 
49. S. R. Phillpot, J. F. Lutsko, D. Wolf, and S. Yip, "Molecular-dynamics study of latticedefect-nucleated melting in silicon," Phys. Rev. B, 40, 2831-2840, 1989 and references therein.

50. A. N. Goldstein, C. M. Echer, and A. P. Alivisatos, "Melting in semiconductor nanocrystals," Science, 256, 1425-1427, 1992.

51. T. Guillot, "Interiors of giant planets inside and outside the solar system," Science, 286, 7277, 1999.

52. C. X. Li, K. Q. Lu, Y. R. Wang, K. Tamura, S. Hosokawa, and M. Inui, "Electronic structure study of liquid germanium based on X-ray-absorption near-edge structure spectroscopy," Phys. Rev. B, 59, 1571-1574, 1999.

53. J. N. Wickham, A. B. Herhold, and A. P. Alivisatos, "Shape change as an indicator of mechanisms in the high-pressure transformations of CdSe nanocrystals," Phys. Rev. Lett., 84, 923-926, 2000.

54. C. C. Chen, A. B. Herhold, C. S. Johnson, and A. P. Alivisatos, "Size dependence of structural metastability in semiconductor nanocrystals," Science, 276, 398-401, 1997.

55. S. H. Tolbert and A. P. Alivisatos, "The wurtzite to rock salt structural transformation in CdSe nanocrystals under high pressure," J. Chem. Phys., 102, 4642-4656, 1995.

56. S. H. Tolbert and A. P. Alivisatos, "Size dependence of a first order solid-solid phase transition: the wurtzite to rock salt transformation in CdSe nanocrystals," Science, 265, 373-376, 1994.

57. D. M. Mittleman, R. W. Schoenlein, J. J. Shiang, V. L. Colvin, A. P. Alivisatos, and C. V. Shank, "Quantum size dependence of femtosecond electronic dephasing and vibrational dynamics in CdSe nanocrystals," Phys. Rev. B, 49, 14435, 1994.

58. R. J. Hemley, H. K. Mao, G. Y. Shen, J. Badro, P. Gillet, M. Hanfland, and D. Hauserman, "X-ray imaging of stress and strain of diamond, iron, and tungsten at megabar pressures,", 276, 1242-1245, 1997.

59. I. S. Elfimov, V. I. Anisimov, and G. A. Sawatzky, "Orbital ordering, Jahn-Teller distortion and anomalous X-ray scattering in manganates," Phys. Rev. Lett., 82, 4264, 1999.

60. Y. Murakami, J. P. Hill, D. Gibbs, M. Blume, I. Koyama, M. Tanaka, H. Kawata, T. Arima, Y. Tokura, K. Hirota, and Y. Endoh, "Resonant X-ray scattering from orbital ordering in $\mathrm{LaMnO}_{3}$," Phys. Rev. Lett., 81, 582, 1998.

61. S. Mori, C. H. Chen, and S.-W. Cheong, "Pairing of charge-ordered stripes in (La,Ca) $\mathrm{MnO}_{3}$," Nature, 392, 6675, 1998.

62. J. Stohr, "NEXAFS Spectroscopy," in Springer Series in Surface Sciences, G. Ertl, R. Gomer, D. L. Mills, and H. K. V. Lotsch, Eds. Heidelberg: Springer-Verlag, 1992.

63. M. Quijada, J. Cerne, J. R. Simpson, H. D. Drew, K. H. Ahn, A. J. Millis, R. Shreekala, R. Ramesh, M. Rajeswari, and T. Venkatesan, "Optical conductivity of manganites: Crossover from Jahn-Teller small polaron to coherent transport in the ferromagnetic state," Phys. Rev. $B, \mathbf{5 8}, 16093,1998$.

64. A. Z. Szarka, N. Pugliano, D. K. Palit, and R. M. Hochstrasser, "Vibrational coherence in the solution phase photoisomerization reaction of cis-stilbene," Chem. Phys. Lett, , 240, 2530, 1995.

65. R. A. Mathies, C. H. Brito Cruz, W. T. Pollard, and C. V. Shank, "Direct observation of the femtosecond excited-state cis-trans isomerization in bacteriorhodopsin," Science, 777-779, 1988. 
66. R. W. Schoenlein, L. A. Peteanu, R. A. Mathies, and C. V. Shank, "The first step in vision: Femtosecond isomerization of rhodopsin," Science, 254, 412, 1991.

67. L. A. Peteanu, R. W. Schoenlein, Q. Wang, R. A. Mathies, and C. V. Shank, "The first step in vision occurs in femtosecond: Complete blue and red spectral shifts," Proc. Natl. Acad. Sci. USA, 90, 11762-11766, 1993.

68. Q. Wang, L. A. Peteanu, R. W. Schoenlein, R. A. Mathies, and C. V. Shank, "Vibrationally coherent photochemistry in the femtosecond primary event of vision," Science, 266, 422424, 1994.

69. M. H. Vos, F. Rappaport, J.-C. Lambry, J. Breton, and J. L. Martin, "Visualization of coherent nuclear motion in a membrane protein by femtosecond spectroscopy," Nature, 363, 320-325, 1993.

70. N. H. Damrauer, G. Cerullo, A. Yeh, T. R. Boussie, C. V. Shank, and J. K. McCusker, "Femtosecond dynamics of excited-state evolution in $\left[\mathrm{Ru}(\mathrm{bpy})_{3}\right]^{2+}$," Science, 275, 54-57, 1997.

71. N. F. Scherer, D. M. Jonas, and G. R. Fleming, "Femtosecond wave packet and chemical reaction dynamics of iodine in solution: Tunable probe study of motion along the reaction coordinate," J. Chem. Phys., 99, 153-167, 1993.

72. U. Banin and S. Ruhman, "Ultrafast photodissociation of $\mathrm{I}_{3}{ }^{-}$- coherent photochemistry in solution," J. Chem. Phys., 98, 4391-4403, 1993.

73. L. Zhu, T. Sage, and P. M. Champion, "Observation of coherent reaction dynamics in heme proteins," Science, 266, 629-632, 1994.

74. B. Bagchi and G. R. Fleming, "Dynamics of activationless reactions in solution," J. Phys. Chem., 94, 9-20, 1990.

75. R. J. Sension, S. T. Repinec, A. Z. Szarka, and R. M. Hochstrasser, "Femtosecond laser studies of the cis-stilbene photoisomerization reactions," J. Chem. Phys., 98, 6291-6315, 1993.

76. W. Fuss, K. L. Kompa, T. Schikarski, W. E. Schmid, and S. A. Trushin, "Ultrafast photoisomerization and $S_{1} / S_{0}$ internal conversions in organic and inorganic molecules: evidence for easily accessible conical intersections," in Ultrafast Phenomena XI, SpringerVerlag Series in Chemical Physics, T. Elsaesser, J. G. Fujimoto, D. A. Wiersma, and W. Zinth, Eds. Berlin: Springer-Verlag, 1998, pp. 615-617.

77. J. K. McCusker, K. N. Walda, R. C. Dunn, J. D. Simon, D. Magde, and D. N. Hendrickson, "Subpicosecond "MLCT- ${ }^{5} \mathrm{~T}_{2}$ intersystem crossing of low-spin polypyridyl ferrous complexes," J. Am. Chem. Soc., 115, 298-307, 1993.

78. H.-R. Chang, J. K. McCusker, H. Toftlund, S. R. Wilson, A. X. Trautwein, H. Winkler, and D. N. Hendrickson, "(Tetrakis(2-pyridylmethyl)ethylenediamine)iron(II) perchlorate, the first rapidly interconverting ferrous spin-crossover complex," J. Am. Chem. Soc., 112, 6814-6827, 1990.

79. C. C. Wu, J. Jung, P. K. Ganzel, P. Gütlich, and D. N. Hendrickson, "LIESST effect studies of Iron(II) spin-crossover complexes with phosphine ligands: Relaxation kinetics and effects of solvent molecules," Inorg. Chem., 36, 5339-5347, 1997 and references therein.

80. J. E. Monat and J. K. McCusker, "Femtosecond excited-state dynamics of a $\mathrm{Fe}(\mathrm{II})$ polypyridyl solar cell sensitizer model," J. Am. Chem. Soc., 122, 4092-4097, 2000.

81. G. Sankar, J. M. Thomas, V. Varma, G. U. Kulkarni, and C. N. R. Rao, "An investigation of the first-order spin-state transition in $\mathrm{Fe}(\mathrm{Phen})_{2}(\mathrm{NCS})_{2}$ EXAFS and infrared spectroscopy," Chem. Phys. Lett., 251, 79-83, 1996. 
82. N. V. Bausk and S. B. Erenburg, "EXAFS study of the spin transition effect on the spatial and electronic structure of Fe(II) complexes with triazoles," J. Struct. Chem., 36, 925-931, 1995.

83. A. Michalowicz, J. Moscovici, B. Ducourant, D. Cracco, and O. Kahn, "EXAFS and x-ray power diffraction studies of the spin transition molecular materials $\left[\mathrm{Fe}(\mathrm{Htrz})_{2}(\operatorname{trz})\right]\left(\mathrm{BF}_{4}\right)$ and $\left[\mathrm{Fe}(\mathrm{Htrz})_{3}\right]\left(\mathrm{BF}_{4}\right)_{2} \mathrm{H}_{2} \mathrm{O}$," Chem. Mater., 7, 1833-1842, 1995.

84. Q. Liu, J.-K. Wang, and A. H. Zewail, "Femtosecond dynamics of dissociation and recombination in solvent cages," Nature, 364, 427-430, 1993.

85. B. J. Schwartz, J. C. King, J. Z. Zhang, and C. B. Harris, "Direct femtosecond measurements of single collision dominated geminate recombination times of small molecules in liquids," Chem. Phys. Lett., 203, 503-508, 1993.

86. C. B. Harris, D. E. Smith, and D. J. Russel, "Vibrational relaxation of diatomic molecules in liquids," Chem. Rev., 90, 481-488, 1990.

87. D. E. Smith and C. B. Harris, "Studies of chemical reactivity in the condensed phase. III. Direct measurement of predissociation and geminate recombination times for Iodine in solution," J. Chem. Phys., 87, 2709, 1987.

88. P. Bado, D. C, D. Magde, K. R. Wilson, and M. M. Malley, "Molecular dynamics of chemical reactions in solution: Experimental picosecond transient spectra for $\mathrm{I}_{2}$ photodissociation," J. Chem. Phys., 80, 5531-5538, 1984.

89. I. Benjamin, U. Banin, and S. Ruhman, "Photodissociation of I in ethanol - a molecular dynamics study," J. Chem. Phys., 98, 8337-8340, 1993.

90. U. Banin, R. Kosloff, and S. Ruhman, "Vibrational relaxation of nascent diiodide ions studied by femtosecond transient resonance impulsive stimulated Raman scattering (TRISRS) - experiment and simulation," Chem. Phys., 183, 289-307, 1994.

91. D. A. V. Kliner, J. C. Alfano, and P. F. Barbara, "Photodissociation and vibrational relaxation of $\mathrm{I}_{2}^{-}$in ethanol," J. Chem. Phys., 98, 5375-5389, 1993.

92. J. C. Alfano, Y. Kimura, P. K. Walhout, and P. F. Barbara, "Photodissocation and vibrational relaxation of $\mathrm{I}_{2}^{-}$in water, ethanol, and ethanol-D(1)," Chem. Phys., 175, 147$155,1993$.

93. V. Vaida and J. D. Simon, "The photoreactivity of chlorine dioxide," Science, 268, 14431448, 1995.

94. J. A. Poulsen, C. L. Thomsen, S. R. Keiding, and J. Thøgersen, "Vibrational relaxation of $\mathrm{ClO}_{2}$ in water," J. Chem. Phys., 108, 8461-8471, 1998.

95. M. J. Philpott, S. Charalambous, and P. J. Reid, "Comparison of chlorine dioxide photochemistry in acetonitrile and water using subpicosecond pump-probe spectroscopy," Chem. Phys. Lett., 281, 1-9, 1997.

96. Y. J. Chang and J. D. Simon, "Ultrafast dynamics of chlorine dioxide photochemistry in water studied by femtosecond transient absorption spectroscopy," J. Phys. Chem., 100, 6406-6411, 1996.

97. A. Baltuska, M. F. Emde, M. S. Pshenichnikov, and D. A. Wiersma, "Early-time dynamics of the photoexcited hydrated electron," J. Phys. Chem A, 103, 10065-10082, 1999.

98. K. Yokoyama, C. Silva, D. H. Son, P. K. Walhout, and P. F. Barbara, "Detailed investigation of the femtosecond pump-probe spectroscopy of the hydrated electron," $J$. Phys. Chem. A, 102, 6957-6966, 1998. 
99. C. Silva, P. K. Walhout, P. J. Reid, and P. F. Barbara, "Detailed investigations of the pump-probe spectroscopy of the equilibrated solvated electron in alcohols," J. Phys. Chem. A, 102, 5701-5707, 1998.

100. J. A. Kloepfer, V. H. Vilchiz, V. A. Lenchenkov, and S. E. Bradforth, "Femtosecond dynamics of photodetachment of the iodide anion in solution: resonant excitation into the charge-transfer-to-solvent state," Chem. Phys. Lett., 298, 120-128, 1998.

101. M. Assel, R. Laenen, and A. Laubereau, "Dynamics of excited solvated electrons in aqueous solution monitored with femtosecond-time and polarization resolution," J. Phys. Chem. A, 102, 1998.

102. Y. Gauduel, M. Sander, and H. Gelabert, "Ultrafast reactivity of IR-excited electron in aqueous ionic solutions," J. Phys. Chem. A, 102, 7795-7803, 1998.

103. N. Chandrasekhar and P. Krebs, "The spectra and the relative yield of solvated electrons produced by resonant photodetachment of iodide anion in ethylene glycol in the temperature range $296<=\mathrm{T}<=453 \mathrm{~K}$," J. Chem. Phys., 112, 5910-5914, 2000.

104. V. Srajer, T. Y. Teng, U. T., P. C., Z. Ren, S. Adachi, W. Schildkamp, D. Bourgeois, M. Wulff, and K. Moffat, "Photolysis of the carbon monoxide complex of myoglobin nanosecond time-resolved crystallography," Science, 274, 1726-1729, 1996.

105. J. B. Wittenberg and D. W. Kraus, "Hemoglobins of eukaryote/prokaryote symbioses," in Structure and Function of Invertebrate Oxygen Binding Proteins, S. N. Vinagradov and O. H. Knapp, Eds. New York: Springer-Verlag, 1991, pp. 225-232.

106. K. R. H. Read, Bio. Bulletin, 123, 605-617, 1962.

107. M. Rizzi, J. B. Wittenberg, A. Coda, M. Fasano, P. Ascenzi, and M. Bolognesi, "Structure of the sulfide-reactive hemoglobin from the clam Lucina pectinata, crystallographic analysis at $1.5 \AA$ A resolution," J. Mol. Biol., 244, 86-98, 1994.

108. P. Eisenberger, R. G. Shulman, B. M. Kincaid, G. S. Brown, and S. Ogawa, "Extended xray absorption fine structure determination of iron nitrogen distances in hemoglobin," Nature, 274, 30-34, 1978.

109. M. Piancastelli, "The neverending story of shape resonances," J. Elect. Spec. and Rel. Phenom., 100, 167-190, 1999.

110. C. B. Harris, E. P. Ippen, G. A. Mourou, and A. H. Zewail, "Ultrafast Phenomena VII," in Springer Verlag Series in Chemical Physics, vol. 53, V. I. Goldanski, F. P. Shafer, and J. P. Toennies, Eds. Berlin: Springer Verlag, 1990.

111. L. D. Noordam and R. R. Jones, "Probing Rydberg electron dynamics," J. Mod. Opt., 44, 2515-2532, 1997.

112. I. M. Waller and J. Hepburn, J. Chem. Phys., 88, 6658, 1988.

113. B. Kim, C. P. Schick, and P. M. Weber, "Time-delayed two-color photoelectron spectra of aniline, 2-aminopyridine, and 3-aminopyridine - snapshots of the nonadiabatic curve crossings," J. Chem. Phys., 103, 6903-6913, 1995.

114. N. Berrah, B. Langer, A. A. Wills, E. Kukk, J. D. Bozek, A. Farhat, and J. Gorczyca, "High-resolution angle-resolved measurements in atoms and molecules using advanced photoelectron spectroscopy at the ALS," J. Elect. Spec. and Rel. Phenom., 103, 1-11, 1999.

115. I. Fischer, D. M. Villeneuve, M. J. J. Vrakking, and A. Stolow, "Femtosecond wave-packet dynamics studied by time-resolved zero-kinetic energy photoelectron spectroscopy," $J$. Chem. Phys., 102, 5566-5569, 1995. 
116. M. Seel and W. Domcke, "Time-resolved ionization spectroscopy of ultrafast internalconversion dynamics on polyatomic molecules - theory and computational studies," $J$. Chem. Phys., 95, 7806-7822, 1991.

117. W. Radloff, V. Stert, T. Freudenberg, I. V. Hertel, C. Jouvet, C. Dedonder-Lardeux, and D. Solgadi, "Internal conversion in highly excited benzene and benzene dimer: femtosecond time-resolved photoelectron spectroscopy," Chem. Phys. Lett., 281, 20-26, 1997.

118. L. C. Zhu, V. Kleiman, X. N. Li, S. P. Lu, K. Trentelman, and R. J. Gordon, "Coherent laser control of the product distribution obtained in the photoexcitation of HI," Science, 270, 77-80, 1995.

119. M. Lewenstein, P. Balcou, M. Yu Ivanov, A. L'Huiller, and P. B. Corkum, "Theory of High Harmonic Generation by Low Frequency Laser Fields," Phys. Rev. A, 49, 2117-2132, 1994.

120. C. E. Clayton, K. A. Marsh, A. Dyson, M. Everett, A. Lai, W. P. Leemans, R. Williams, and C. Joshi, "Ultrahigh-Gradient Accelration of Injected Electrons by Laser-Excited Relativistic Electron Plasma Waves," Phys. Rev. Lett., 70, 37-40, 1993.

121. B. E. Lemoff, G. Y. Yin, C. L. G. III, C. P. J. Barty, and S. E. Harris, "Demonstration of a 10-Hz Femtosecond-Pulse_driven XUV Laser at $41.8 \mathrm{~nm}$ in Xe 1X," Phys. Rev. Lett., 74, 1574-1577, 1995.

122. R. R. Freeman, P. H. Bucksbaum, H. Milchberg, S. Darak, D. Schumacher, and M. E. Geusic, "Above-threshold ionization with subpicosecond laser pulses," Phys. Rev. Lett., 59, 1092-1095, 1987.

123. P. B. Corkum, N. H. Burnett, and F. Brunel, "Above-Threshold Ionization in the LongWavelength Limit," Phys. Rev. Lett., 62, 1259-1262, 1989.

124. E. Mevel, P. Breger, R. Trainham, G. Petite, and P. Agostini, "Atoms in strong optical fields: Evolution from multiphoton to tunnel ionization," Phys. Rev. Lett., 70, 406-409, 1993.

125. A. Cionga, V. Florescu, A. Maquet, and R. Taïeb, "Target dressing effects in laser-assisted x-ray photoionization," Phys. Rev. A, 47, 1830-1840, 1993.

126. J. M. Schins, P. Breger, P. Agostini, R. C. Constantinescu, H. G. Muller, G. Grillon, A. Antonetti, and A. Mysyrowicz, "Observation of laser-assisted Auger decay in argon," Phys. Rev. Lett., 73, 2180-2183, 1994.

127. T. E. Glover, R. W. Schoenlein, A. H. Chin, and C. V. Shank, "Observation of laser assisted photoelectric effect and femtosecond high order harmonic radiation," Phys. Rev. Lett., 76, 2468, 1996.

128. M. F. Van Leeuwen, S. Papademetriou, and J. C. R. Stroud, "Autler-Townes effect for an atom in a 100\% amplitude-modulated laser field. I. A dressed-atom approach," Phys. Rev. A, 53, 990-996, 1996.

129. D. Cubaynes, J. M. Bizau, F. J. Wuilleumier, B. Carre, and F. Gounand, "Inner-shell photoionization in an excited atom: many-electron effects and partial cross section in $2 p^{6} 3 p^{2} \mathrm{P}_{3 / 2}$ Sodium atoms," Phys. Rev. Lett., 63, 2460, 1989.

130. J. M. Bizau, D. Cubaynes, P. Gerard, F. J. Wuileumier, J. L. Picque, D. L. Ederer, B. Carre, and G. Wendin, "Experimental and theoretical determinations of the 5d photoionization cross section in laser-excited barium atoms between 15 and $150 \mathrm{eV}$ photon energy," Phys. Rev. Lett., 57, 306, 1986.

131. M. Ferray, F. Gounand, P. D'Oliveira, P. R. Fournier, D. Cubaynes, J. M. Bizau, T. J. Morgan, and F. J. Wuilleumier, "Three-step excitation of highly excited autoionizing states 
in atomic sodium by use of laser beams and synchrotron radiation," Phys. Rev. Lett., 59, 2040, 1987.

132. C. Limborg, "Present time structure properties of storage ring based X-ray sources," presented at Time Structrue of X-ray Sources and its Applications, San Diego, CA, SPIE, 1998.

133. K.-J. Kim, "Physics of Particle Accelerators," presented at AIP Conference Proceedings 184, AIP, 1989.

134. D. E. Alferov, Y. A. Bushmakov, K. A. Belovintsev, E. G. Bessonov, and P. A. Cherenkov, Particle Accel., 9, 223, 1979.

135. "1-2 GeV Synchrotron Radiation Source - Conceptual Design Report," Lawrence Berkeley Laboratory LBL PUB-5172 Rev., July 1986.

136. S. Backus, C. G. Durfee, M. M. Murnane, and H. C. Kapteyn, "High power ultrafast lasers," Rev. Sci. Inst., 69, 1207-1223, 1998.

137. M. J. W. Rodwell, D. M. Bloom, and K. J. Weingarten, "Subpicosecond laser timing stabilization," IEEE J. Quant. Electron., 25, 817, 1989.

138. For x-rays, the relatively small collection angle of the beamline is more than sufficient to accurately image the source (electron beam) without being limited by diffraction.

139. E. Wallen, G. LeBlanc, and M. Eriksson, "The MAX-Wiggler, a cold bore superconducting wiggler with 473.5 T poles," Nucl. Inst. and Meth. A, 467-468, 118-121, 2001.

140. "Superbend Conceptual Design Report," Lawrence Berkeley National Laboratory LBNL Pub. 5457, April 2000.

141. "X-ray Data Booklet," Lawrence Berkeley National Laboratory LBNL Pub. 490 Rev. 2, January 2001.

142. A. Zholents and W. Decking, "Non-Gaussian tails at the Advanced Light Source," Lawrence Berkeley National Laboratory LBNL Report No. 45392, 1999.

143. E. L. Church and P. Z. Takacs, in Handbook of Optics, vol. 1: McGraw-Hill, Inc., 1975, pp. 7.1-7.14.

144. H. A. Padmore, M. R. Howells, S. Irick, T. Renner, R. Sandler, and Y.-M. Koo, "Some new schemes for producing high-accuracy elliptical X-ray mirrors by elastic bending," presented at Optics for High-Brightness Synchrotron Radiation Beamlines II, SPIE Proceedings, 1996.

145. H. Petersen, C. Jung, C. Hellwig, W. B. Peatman, and W. Gudat, "Review of plane grating focusing for soft x-ray monochromators," Rev. Sci. Instrum., 66, 1-14, 1995.

146. M. Sánchez del Río and R. J. Dejus, "XOP: A Multiplatform Graphical User Interface for Synchrotron Radiation Spectral and Optics Calculations,", SPIE Proceedings, 1997.

147. R. Petit, "Electromagnetic Theory of Gratings," in Topics in Current Physics, vol. 22, 1980.

148. D. Strickland and G. Mourou, "Compression of amplified chirped optical pulses," Opt. Comm., 56, 219-221, 1985.

149. Y. Nabekawa, T. Togashi, T. Sekikawa, S. Watanabe, S. Konno, T. Kojima, S. Fujikawa, and K. Yasui, "All-solid-state high-peak-power Ti:sapphire laser system above 5-kHz repetition rate," Appl. Phys. B, 70, S171-S179, 2000.

150. H. Wang, S. Backus, Z. Chang, R. Wagner, K. Kim, X. Wang, D. Umstadter, T. Lei, M. Murnane, and H. Kapteyn, "Generation of 10-W average-power, 40-TW peak-power, 24-fs pulses from a Ti: sapphire amplifier system,” J. Opt. Soc. Am. B, 16, 1790-1794, 1999. 
151. B. Walker, C. Tóth, D. N. Fittinghoff, T. Guo, D.-E. Kim, C. Rose-Petruck, J. A. Squier, K. Yamakawa, K. R. Wilson, and C. P. J. Barty, "A $50 \mathrm{EW} / \mathrm{cm}^{2}$ Ti:sapphire laser system for studying relativistic light-matter interactions," Opt. Exp., 5, 196-202, 1999.

152. High-power continuous wave titanium:sapphire laser: U.S. patent \# 5,247,527

153. P. A. Schulz and S. R. Henion, "Liquid-nitrogen-cooled Ti: $\mathrm{Al}_{2} \mathrm{O}_{3}$ laser," IEEE J. Quantum Electron., 27, 1039-1047, 1991.

154. M. Zavelani-Rossi, F. Lindner, C. Le Blanc, G. Cheriaux, and J. P. Chambaret, "Control of thermal effects for high-intensity Ti : sapphire laser chains," Appl. Phys. B, 70, S193-S196, 2000.

155. J. Z. Yang and B. C. Walker, "0.09-terawatt pulses with a $31 \%$ efficient, kilohertz repetition-rate Ti:sapphire regenerative amplifier," Opt. Lett., 26, 453-455, 2001.

156. S. Backus, R. Bartels, S. Thompson, R. Dollinger, H. C. Kapteyn, and M. M. Murnane, "Sigh-efficiency, single-stage 7-kHz high-average-power ultrafast laser system," Opt. Lett., 26, 465-467, 2001.

157. Z. Chang, A. Rundquist, J. Zhou, M. M. Murnane, and H. C. Kapteyn, "Demonstration of a sub-picosecond x-ray streak camera," Appl. Phys. Lett., 69, 133-135, 1996.

158. Y. Wu and H. Nishimura, "Mini-beta lattice for the femtosecond X-ray beamline at the Advanced Light Source," Lawrence Berkeley National Laboratory LBNL Report (in press), 2000.

159. J. Yu, Z. Jiang, J. C. Kieffer, and A. Krol, "Hard X-ray emission in high intensity femtosecond laser-target interaction," Phys. of Plasmas, 6, 1318-1322, 1999.

160. J. M. Cao, H. Ihee, and A. H. Zewail, "Ultrafast electron diffraction and direct observation of transient structures in a chemical reaction," Proc. Nat. Acad. Sci. USA, 96, 338-342, 1999.

161. J. C. Williamson, J. M. Cao, H. Ihee, H. Frey, and A. H. Zewail, "Clocking transient chemical changes by ultrafast electron diffraction," Nature, 386, 159-162, 1997.

162. G. Mourou and S. Williamson, "Picosecond electron diffraction," Appl. Phys. Lett., 41, 4145, 1982.

163. P. A. Lee, P. H. Citrin, P. Eisenberger, and B. M. Kincaid, "Extended x-ray absorption fine structure - its strengths and limitation as a structural tool," Rev. Mod. Phys., 53, 769-806, 1981.

164. V. P. Krinov, H. R. Reiss, and B. M. Smirnov, Radiative Processes in Atomic Physics. New York: John Wiley \& Sons, 1997. 
Ultrafast X-ray Science Facility at the Advanced Light Source

Biographical Information 